\title{
Indagine su alcuni terremoti con epicentro nell'area Mediterranea
}

\author{
Valutazione dell'attendibilità delle dromocrone $P_{n}$ \\ calcolate per i terremoti avvenuti nella Sicilia Occidentale
}

(Investigation on some earthquakes originating in the Mediterannean Area.
The reliability's estimation of Pn (ravel-times computed for the
earthquakes occurred in West-Sicily)

L. Mircelul - G. Paxyoccimi

Ricevuto il 29 Novenbre 1974

Riassunto. - Nol presente lavoro si è voluto effettuare un controllo sull'attendibilità dei risultati ottenuti per i tempi di tragitto delle $P_{n}$ provenienti dalla sicilia occidentale.

A questo seopo è stata svolta un'accurata indagine su 25 terremoti con epicentro nell'area Mediterranea: nli elementi fondamentali da cui si è partiti per effettuare il controllo, sono quelli forniti dall'International Seismological (centre $\left(p_{0}, \lambda_{0}, h \pm \delta h, I I \pm \delta I\right.$, sD) $)$ che sono stati fatti oggetto di preliminari critiche e riscussioni.

Per ciascun terremoto sono stati calcolati i $l_{r n}, t_{P^{\prime} n_{1}}, t_{r^{\prime} u_{2}}$ delle stazioni che lo hanno registrato (fino a $1 \leqslant 20^{\circ}$ ): il calcolo è stato eseguito con le dromocrone (relative al una o due profondita ipocentrali) ricavate dai precedenti studi sui terremoti della sicilia oecidentale. I risulati del controllo sono stati poi classificati in $A, B, C, D$ secondo un criterio di suddivisione in classi di attendibilita da noi suggerito in una nota precedente.

Gli A, B, C, D sono stati opportunamente elaborati in quattro serie successive: la tabella $n$. $l$ e la fig. 4 no sintetizzano i risultati.

L'indagine condotta porta a ritenere attenribili le curve $l_{P n}, l_{P_{1}}, l_{n_{n_{2}}}$, calcolate por i terremoti della sicilia: queste curve sono applicalili in maniera soddisfacente a tutti i teremoti provenienti dall'area Mediterranca e le rendono preferibili a quelle in uso per l'Italia, più valicle queste ultime, per i terremoti con epicentro in territorio italiano. Dall'indagine condota risulta anche che i terremoti dellarea Ifediterranea sembrano avvenire a profondita generalmente più elevate di quelli italiani centro-settentrionali. 
SUMmary. - The object of this work has been performed to check the reliability of the results obtained for the $P_{n}$ travel-times arising from western sicily.

For this purpose a careful investigation on 25 arthquakes originating in the Mediterranean area has been developed: the hasic parameters used to carry out the check, have been the ones computed by the International Seismological Centre $\left(\varphi_{0}, \lambda_{0}, h \pm \delta h, H \pm \delta I I\right.$, SI) ) previously subjected to a critical examination. For each earthquake $t_{P n}, t_{P n_{1}}$, and $t_{P n_{2}}$, of the recording stations (up to $A \leqslant 20^{\circ}$ ) have been calculated by the traveltimes (with respect to one or two focus deptlas) previously studied and computed for earthquakes in western Sicily $\left({ }^{3}\right)$. The results have been then classified under $A, B, C$, and $I$ according to a criterion of subdivision in classes of reliability suggested by us in another work (4). $A, B, C$, and D have been suitably elaborated in four successive series; both the Table 1 and figure 4 synthetize these results.

The investigation supports the reliability of the $t_{P n}, t_{P n_{1}}$, and $t_{P n_{2}}$, travel-times calculated for the Sicilian earthquakes: these travel-times can satisfactorily be applied to all earthquakes originating in the Mediterranean area and are to be preferred to the travel-times employed for Italy which, however, are to be deemed more suitable for earthquakes whose epicentre is in Italian territory. This investigation also points out that the earth. quakes in Ylediterranean area generally seem to oceur at a greater depth than those in middle and northern Italy.

\section{RICHIMI - SCOPO DELLA RICERCA E GENERAIITÀ}

Nello studio sui tempi di tragitto delle $P_{n}$ provenienti dalla Sicilia occ. $\left({ }^{3}\right)$ siamo pervenuti ad una serie di equazioni relative a varie profondita ipocentrali comprese fra 30 e $60 \mathrm{~km}$ e ne abbiamo riportate le tabelle numeriche calcolate ogni $20 \mathrm{~km}$ fino a $\Delta=20^{\circ}$.

Ricordiamo che quello studio è stato conclotto con lo scopo di analizzare il comportamento delle onde sismiche entro un'area piuttosto ristretta intorno alla regione italiana e zone immediatamente aliacenti.

Allo scopo di effettuare qualche controllo sull'attendibilità dei risultati conseguiti, abbiamo poi voluto svolgere un'inclagine su alcuni terremoti con epicentro nell'area mediterranea, terremoti di cui si conoscono i dati fondamentali e i tempi d'arrivo delle prime onde individuate dai vari Osservatori europei.

A questo scopo abbiamo scelto 25 terremoti distribuiti in modo da abbracciare l'intera area mediterranea, disposti a ventaglio intorno alle stazioni dell'Italia. Gli epicentri dei 25 terremoti scelti per questo 
controllo sono localizzati nelle seguenti regioni (nell'ordine da W verso E):

Portogallo, Spagna, Maroceo, Algeria, Tunisia, Sicilia occ., II. Mexliterraneo (al largo delle coste dell'Africa), basso Tirreno, Italia Meridionale (Campania), Mare Arriatico (coste Dalmate), Mar Ionio (Coste Calabre), Iugoslavia, Mar Ionio (presso la Grecia), Grecia, Albania, Mediterraneo orientale (al largo di Creta), Creta, Rodi, Turchia, Tegitto (v. fig. 1).

Questi terremoti sono avvenuti prevalentemente negli anni 196970: soltanto tre negli anni precerlenti. I dati ipocentrali di riferimento per eflettuare il nostro controllo, sono stati dedotti dai bollettini sismici dell'ISC (International Seismological Centre) e sono riportati nella parte alta della tabella riassuntiva (v. tab. n. 1). In questa parte abbiamo riportato anche eventuali altre profonditi ipocentrali ed eventuali altri tempi origine ealcolati da centri diversi dall'ISC specialmente quando questi dati differiscono tra loro in modo da fornire elementi di discussione e di incertezze.

Diciamo subito però che ogmi nostro controllo ’̀ effettuato rispetto ai dati forniti dall'ISC.

Un'attenzione particolare è stata rivolta agli errori $|\delta \Pi|$ riportati nel ealcolo dei tempi origine $H$, all'errore standard SD, ed ai residui $(O-C) \equiv\left[t_{\text {oss }} t_{\text {calc. }}\right]$ riportati nel bollettino a fianeo di ciascuna stazione; e ciò perché, come abbiamo avuto modo di riscontrare ampiamente, e come del resto è ovvio, variazioni di $t_{P_{n}}$ anche piccole incirlono in maniera notevole sugli altri elementi del terremoto [v. lig. 3].

\section{Metodo segutTo NeLL'TNDAGINe.}

Premesso che dato lo scopo della nostra indagine abbiamo sempre limitato la nostra attenzione alle stazioni con $\Delta$ contenute entro $20^{\circ}$ dall'epicentro, passiamo ad esporre il procedimento seguito in questo esame.

a) Per ciascuno dei 25 terremoti scelti abbiamo considerato tutte le stazioni riportate dal bollettino ISC (fino a $A=20^{\circ}$ ) e ne abbiamo calcolati i tempi di tragitto $\left(t_{P n}-H\right)$ in base ai tempi di registrazione $t_{P n}$ riportati sul bollettino stesso, con riferimento al tempo origine $H$ calcolato dall'ISC; 
b) fissata poi l'attenzione sul gruppo di dromocrone ( $P_{n \text { genera1, }}$ $\left.P_{n 1}, P_{n 2}\right)\left({ }^{3}\right)$ relative alla profondita ipocentrale più prossima alla $\hbar$ calcolata dall'ISC, per ogni stazione abbiamo dedotto le distanze epicentrali corrispondenti ai tempi di tragitto $t_{P n(g e n .)}, t_{P n_{1}}, t_{P n_{2}}$;

c) chiamate queste distanze $A_{\text {tabb. }}$ le abbiamo confrontate con

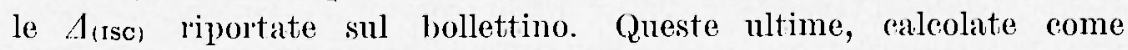
distanze geocentriche tra il punto-Epicentro e il punto-Stazione, costituiscono un valido termine di confronto, talche la diflerenza tra le due distanze $\left[\Delta_{(t a b)}-\Delta_{(\text {ISc })}\right]$ dà una inclicazione della validita della dromocrona usata;

d) quando peró il bollettino riportava valori di $h$ alquanto dissimili tra loro, abbiamo tentato il controllo anche con più dromocrone per verlere quale $h$ si alattava meglio. Piǹ controlli sono stati fatti anche quando l'errore calcolato dell'h $h_{\text {ISc }}$ era troppo alto (v. 1). es. terr. n. 9);

e) per ogni terremoto inoltre, come precedentemente detto, abbiamo sottoposto ad un attento esame i residui (O-C) di ogni singola stazione (v. tab. n. 2 dei resirlui). In certi casi i valori che ivi compaiono sono troppo elevati per poter rientrare nei normali errori di osservazione, e la loro utilizazione può disorientare nelle conchsioni.

Nel nostro controllo abbiamo quindi ritenuto utile tener presenti anche queste indicazioni allo scopo di poter valutare le incertezze che ne derivano.

Abbiamo perció convenuto di considerare i resirlui $(\mathrm{O}-\mathrm{C})<|2 \mathrm{~s}, 4|$ come contenuti entro i normali limiti di tolleranza, e di inclicare quelli più elevati con tre contrassegni diversi, a seconda del valore:

con $\dot{\mathrm{S}}$ le stazioni per le quali $(\mathrm{O}-\mathrm{C})$ è compreso tra $|2 \mathrm{~s}, 4|$ e $|3 \mathrm{~s}, 9|$

con $\ddot{S}$ le stazioni per le quali (O-C) è compreso tra $|4 s, 0|$ e $|6 \mathrm{~s}, 2|$ con $\dot{\mathrm{S}}$ le stazioni per le quali (O-C) è compreso tra $|6 \mathrm{~s}, 2|$ e $\left|10^{\mathrm{s}}\right|$

eschulendo senz'altro quelle con $\left(\mathrm{O}-(\mathrm{)})>\left|10^{\mathrm{s}}\right|\right.$. Riteniamo però che anche tra le stazioni contrassegnate come sopra ve ne siano alcume che, pur avendo fornito la lettura delle prime onde, questa non sia riferibile alle $P_{n}$ o altrimenti sia errata per qualche altro motivo (errore di lettura, errore nella correzione tempolale etc.); 


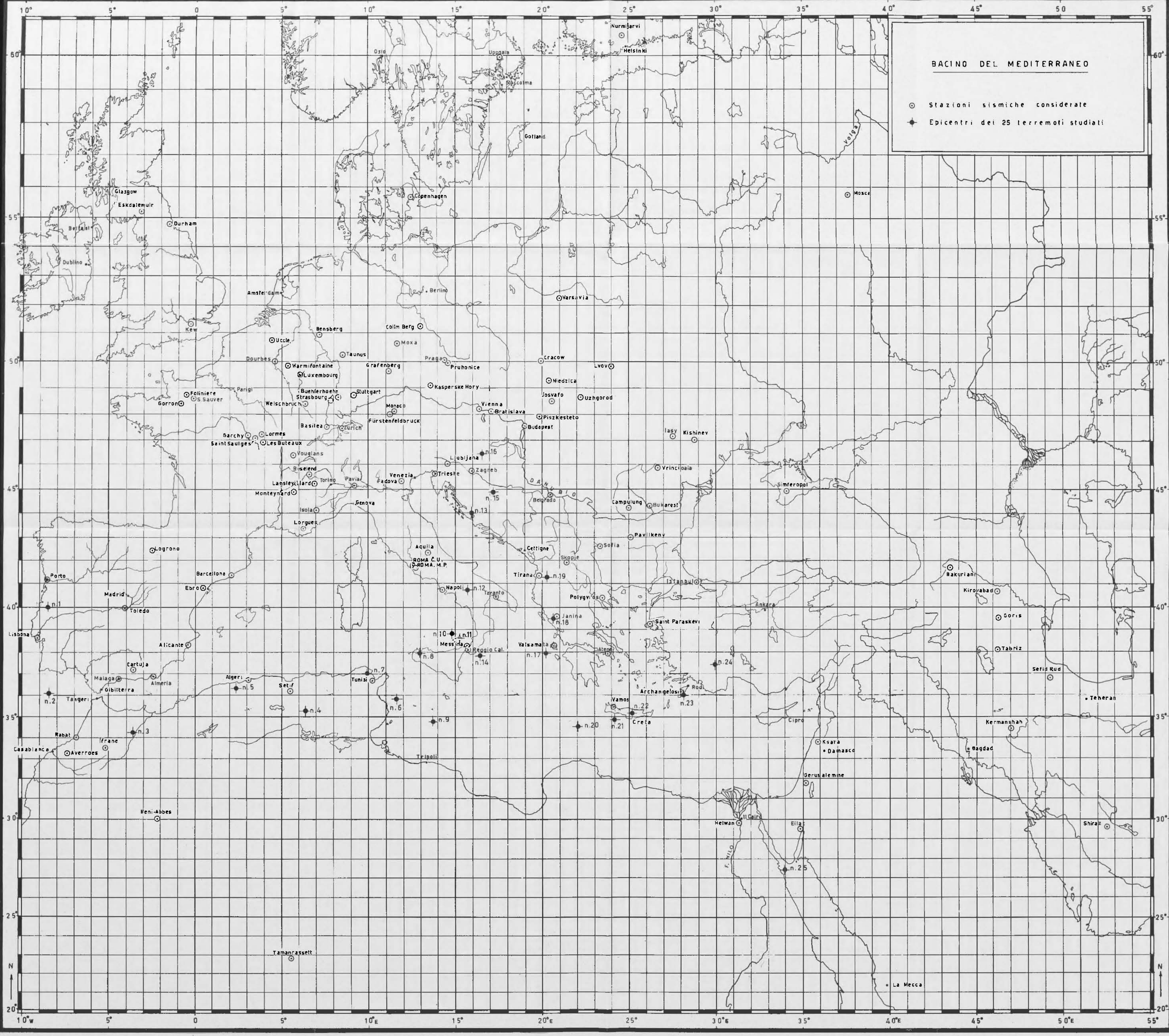


f) ciò premesso, per ciascuma stazione siamo passati come detto, all'esame delle diflerenze

$$
\left[\Delta_{(\text {tab. })}-\Delta_{(\text {Isc })}\right]=\delta .1 .
$$

Partendo dal presupposto che da ma interpretazione diretta di un sismogramma non si possono mai trarre elementi di valulazione $A$ rigorosi, ma ci si deve sempre attendere una approssimazione il cui valore ì funzione della distanza epicentrale, abbiamo suddiviso le difterenze di cui sopra nelle quattro assi di at tendibilita $A, B, C, 1)$ (alelle quali abbiamo trattato nella nota citata (4)), secondo i grafiei della fig. 2 .

In questa maniera l'indicazione fornita da ciascuna stazione viene assegnata ad una classe di attendibilità e cio permette di tralure in termini concisi i risultati delle nostre osservazioni.

Alla classe $A$ apparterranno i dati delle stazioni per le quali la distanza calcolata dalle nostre dromocrone coincide praticamente con quella calcolata dall'ISC (e ció conferma la valiolità della dromocrona usata); alla classe B apparteramno tutti i dati delle stazioni per le quali la coincidenza è meno rigorosa, ma si mantiene pur sempre entro limiti accettabili in campo sperimentale, sicché un alto valore percentuale del termine $(A+B)$, da noi calcolato per ogni controllo, è indizio di valori sorldisfacenti.

Alle classi $\mathrm{C}$ e $\mathrm{D}$ invece verramo assegnati i dati delle stazioni per le quali la discordanza è sensibile o adclirittura intollerabile. Ovviamente in tali classi ritroveremo quasi tutte quelle stazioni che presentavano gia in partenza delle gravi incertezze di tempo (quelle da noi contrassegnate con $\ddot{S}$ e $\dot{\hat{S}})$ mentre a volte quelle indicate con s trovano posto nelle classi migliori. Abbiamo tenuto presente anche lincidenza che le piccole variazioni di tempo hamo sulle distanze, incidenza da noi messa in evidenza nella nota citata e di cui riportiamo il grafico (fig. 3).

Chiariremo quanto sopra con l'applieazione pratica che passiamo an esporre.

FLAT3ORAZIONE DEI DATI: LE QUATTRO SERIE DI CONTROLI.I.

La classificazione dei dati delle stazioni nel morlo suddetto ci ha consentito di elaborare i risultati in modo tale da renderli evidenti e confrontabili tra loro. 


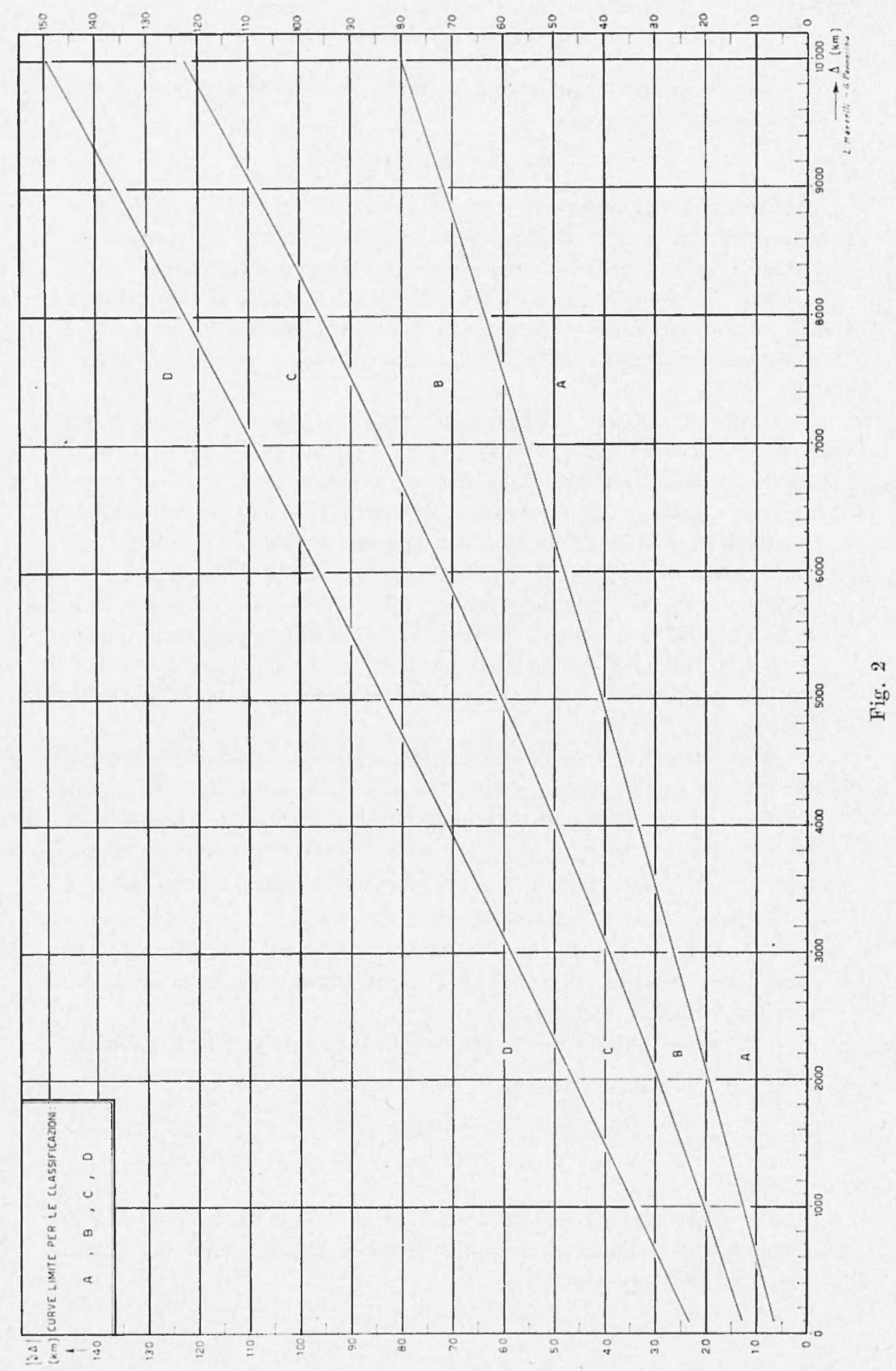




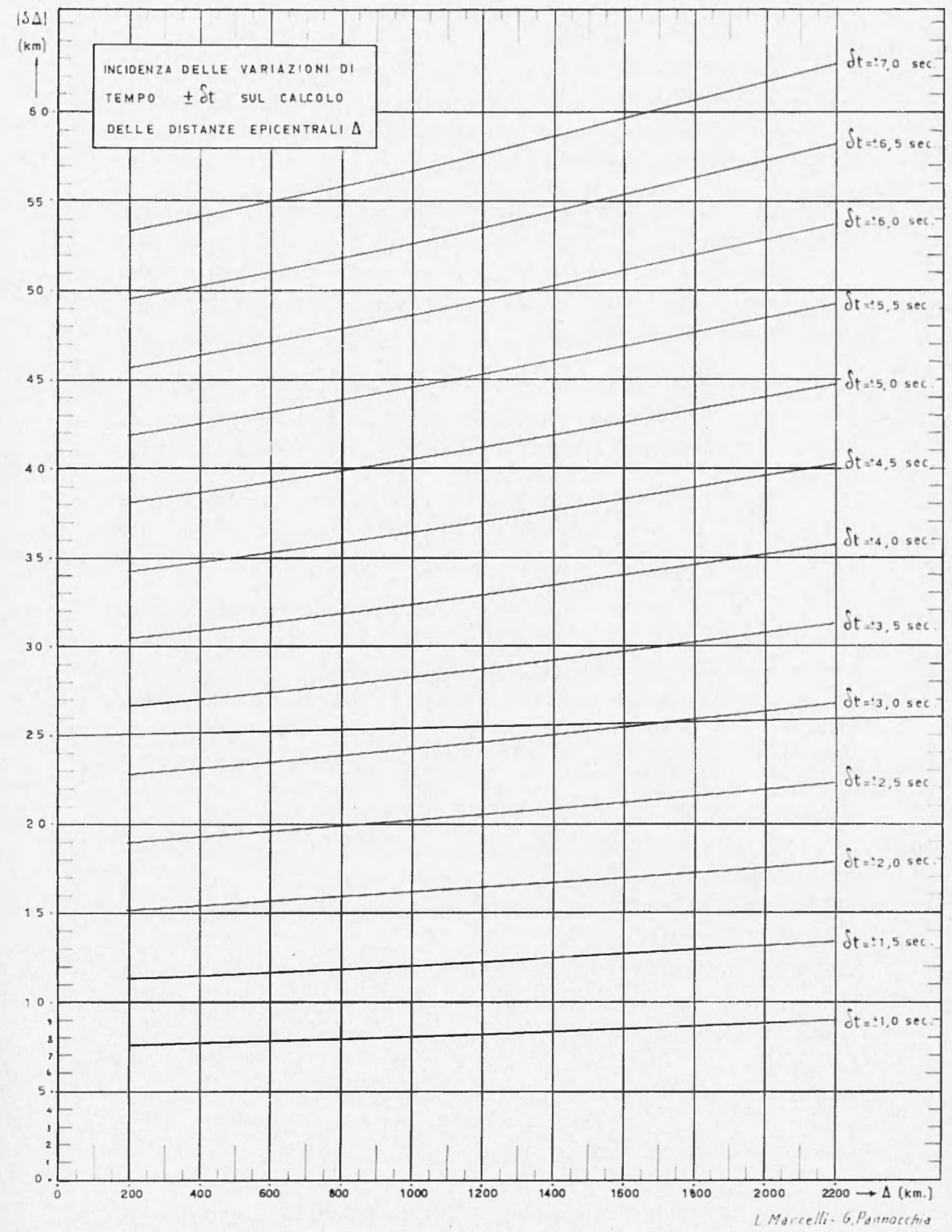

Fig. 3 
Una volta esaurita la preparazione dlei punti $a), b), c), d), e), f$ ) abbiamo procerluto nella seguente maniera:

„li ogni terremoto, e per ciascuna profonditì ipocentrale, abbiamo elaborato $i$ rlati in quattro serie.

Nella $I$ serie abbiamo considerato tutte le $N_{1}$ stazioni rilevate clal bollettino (fino a $\Delta=20^{\circ}$ ), e le prime onde di ciascuna sono state classificate in $\Lambda, B, C, D$ secondo il precedente criterio, quindi abbiamo calcolato le percentuali delle varie chassi corrispondenti rispettivamente alle $P_{n \text { (gen.) }}, P_{n 1}$ e $P_{n 2}$ :

nella II serie gli stessi calcoli sono stati fatti, eschulenulo però tutti i lati che avevano incertezze iniziali $(\dot{\mathrm{S}}, \ddot{\mathrm{S}}, \dot{\overrightarrow{\mathrm{S}}})$. Ise $N$, stazioni residue, considerate più attendibili, danno in genere risultati migliori;

una III serie è stata calcolata quando i tempi origine $I I$ erano stati dati dall'ISC con un errore $\geqslant|2 \mathrm{~s}|$, e in tal caso si è tenuto conto rlelle $\delta \Delta$ secomdo il grafico ali fig. 3 ;

la $I V$ serie infine è stata calcolata chiamamo $P_{n}$ (e conteggiandolo una volta sola), il valore migliore scelto tra la $P_{n 1}$ e la $P_{n 2}$ ricordanclo la ipotizzata esistenza (che sembra comprovata dallattuale analisi) di onde longitudinali propagantisi con velocita lievemente diverse (3)): là dove compaiono le $P_{n 1}$ non possono comparire le $P_{n 2}$ e viceversa (ad ma A di una $P_{n 1}$ spesso corrisponde una B o addirittura una C nella $P_{n 2}$ ) (esempio terr. n. 9). E da notare che i controlli eseguiti con le dromocrone relative ar $h=5 \mathrm{~km}$ e al $h=17,5 \mathrm{~km}$, le quali non hamno distinzioni di $P_{n}$, portano la IV serie ad irlentificasi con la I e la II.

La IV serie è risultata sempre la migliore e ciò darebbe ulteriore credito all'esistenza delle $P_{n 1}$ e $P_{n}{ }^{2}$.

A questo proposito possiamo dire che spesso abbiamo constatato che per alcuni scauti sensibili, quali quelli contrassegnati con $\dot{S}$, facendo i debiti conti, le $P_{n}$ divengono $P_{n 1}$ o $P_{n 2}$ a seconda del segno dei residui.

\section{25 TERREMO'T ANALIZZATI.}

Sarebbe troppo lungo riportare tutta la mole del lavoro svolto in questa analisi. Esporremo, il più concisamente possibile, i risultati 
cui siamo pervenuti per ogni terremoto in particolare, per poi trame delle conchusioni di carattere generale.

Peró riteniamo doveroso, per una mighiore comprensione della tecnica usata, esporre almeno per un terremoto, il procedimento completo.

Per questo scopo abbiamo scelto il terremoto n. 9 che riassume in sé tutte le osservazioni fatte, sicché può servire di esempio per tutti gli altri.

Il terremoto $n .9$. (9 ottobre 1970). Epicentro nel Mar Mediterraneo (Coste dell'Africa)

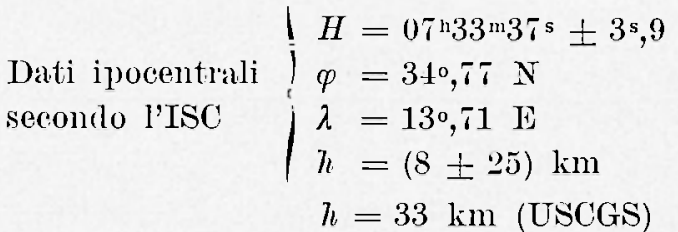

$$
\begin{aligned}
& \mathrm{Mag}=4, \pm \\
& \mathrm{SD}=2 \mathrm{~s}, 78 \text { (su } 78 \text { osservazioni) }
\end{aligned}
$$

[v. tabelle 1 e 3$]$

Analisi della Tabella 3 (del terremoto n. 9) ed elaborazione dei dati:

Per questo terremoto molte sono le incertezze iniziali.

Delle $t 1$ stazioni prese in considerazione fino a $A=20^{\circ}$, venti hamo resichui elevati (v. colomna (O-C) della tabella) e precisamente 10(S), $7(\ddot{\mathrm{S}}), 3(\dot{\mathrm{S}})$, ossia solo 21 stazioni (il $51,2 \%$ ) (larebbero garanzia di attendibilità.

I grandi valori delle correzioni relative al tempo origine $\left(H=07^{n} 33^{\mathrm{m}} 3 \mathrm{r}^{\mathrm{s}} \pm 3^{\mathrm{s}}, 9\right)$ ed alla profondita ipocentrale $(h=(8 \pm 25)$ $\mathrm{km}$ ), il valore elevato dell'errore standard (SD $=2 \mathrm{~s}, 78$ su 78 osservazioni) famno prevelere difficoltà nei controlli.

Dati gli elementi di partenza è stato controllato il comportamento delle dromocrone relative alle due profondita:

$h=5 \mathrm{~km}$ (dromocrona usata per i terremoti italiani, calcolata su uno studio del G. Sasso (l'Italia) $\left(^{1}\right)$

$h=35 \mathrm{~km}$ (serie di dromocrone $\left(P_{n(\mathrm{gen} .)} P_{n 1} P_{n 2}\right)$ calcolate per questa h. nello studio dei terremoti della Sicjlia occidl.) $\left(^{3}\right)$.

Sohematizziamo gli elementi della tabella n. 3 nelle quattro serie di cui abbiamo parlato. 
Nella $I$ serie abbiamo elaborato i dati di tatte le $N_{1}=11$ stazioni considerate oftenendo $\mathrm{i}$ seguenti risultati:

\begin{tabular}{|c|c|}
\hline & $I_{n}$ \\
\hline 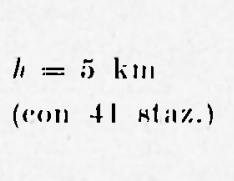 & $\begin{array}{l}\left.\begin{array}{l}1=5 / 41=12 \% \\
B=9 / 41=22 \%\end{array}\right\}(A+B)=34,2 \% \\
1=10 / 41=24,4 \% \\
1)=17+4=41,5 \%\end{array}$ \\
\hline
\end{tabular}

$$
\begin{gathered}
h=35 \mathrm{~km} \\
(\text { coll } 4 \text { I } \mathrm{stct})
\end{gathered}
$$

\begin{tabular}{|c|c|c|}
\hline$I_{n}($ (rron. $)$ & $I^{\prime} n_{1}$ & $I_{n=}$ \\
\hline $\begin{array}{l}\Lambda=17+1=41,5 \% \\
13=12+1=29,3 \% \\
1=5 / 41=12,2 \% \\
1)=7+1=17,1 \% \\
(\Lambda+13)=70,8 \%\end{array}$ & $\begin{array}{l}A=13+1=31,7 \% \\
B=10 /+1=24,4 \% \\
1=11+1=26,8 \% \\
I=7 /+1=17,1 \% \\
(A=13)=56,1 \%\end{array}$ & $\begin{array}{l}A=21 / 4=51,2 \% \\
\mathrm{~B}=4 /+1=9,8 \% \\
(:=1+1=22 \% \\
D=7+1=17,1 \% \\
(A=B)=61 \%\end{array}$ \\
\hline
\end{tabular}

I conti latti con questa serie damon risuldati poso sodelisfacenti, com'era preverlibile.

Ia prof. $h=5 \mathrm{~km}$ sembrerebbe da scartare, mentre per $h=35$ kun anmenta semsibihnente il munero delle stazioni di classe $A$.

Dalla $I I$ serie, ottenuta elaborando le sole $N_{2}=21$ stazioni sonza incertezze iniziali, ci somo da attendersi risultati migliori, almeno per Ima delle due h esaminate.

Ed infatti si hat:

II serie (conl $N_{2}$ stazioni)

$$
\begin{gathered}
h=5 \text { km } \\
(\text { oon } 21 \text { ataz) }
\end{gathered}
$$

\begin{tabular}{|l}
\hline \\
$\mathrm{A}=2 / 21=9,5 \%$ \\
$\mathrm{~B}=7 / 21=33,3 \%$ \\
$\mathrm{C}=7 / 21=33,3 \%$ \\
$\mathrm{D}=5 / 21=23,8 \%$
\end{tabular}




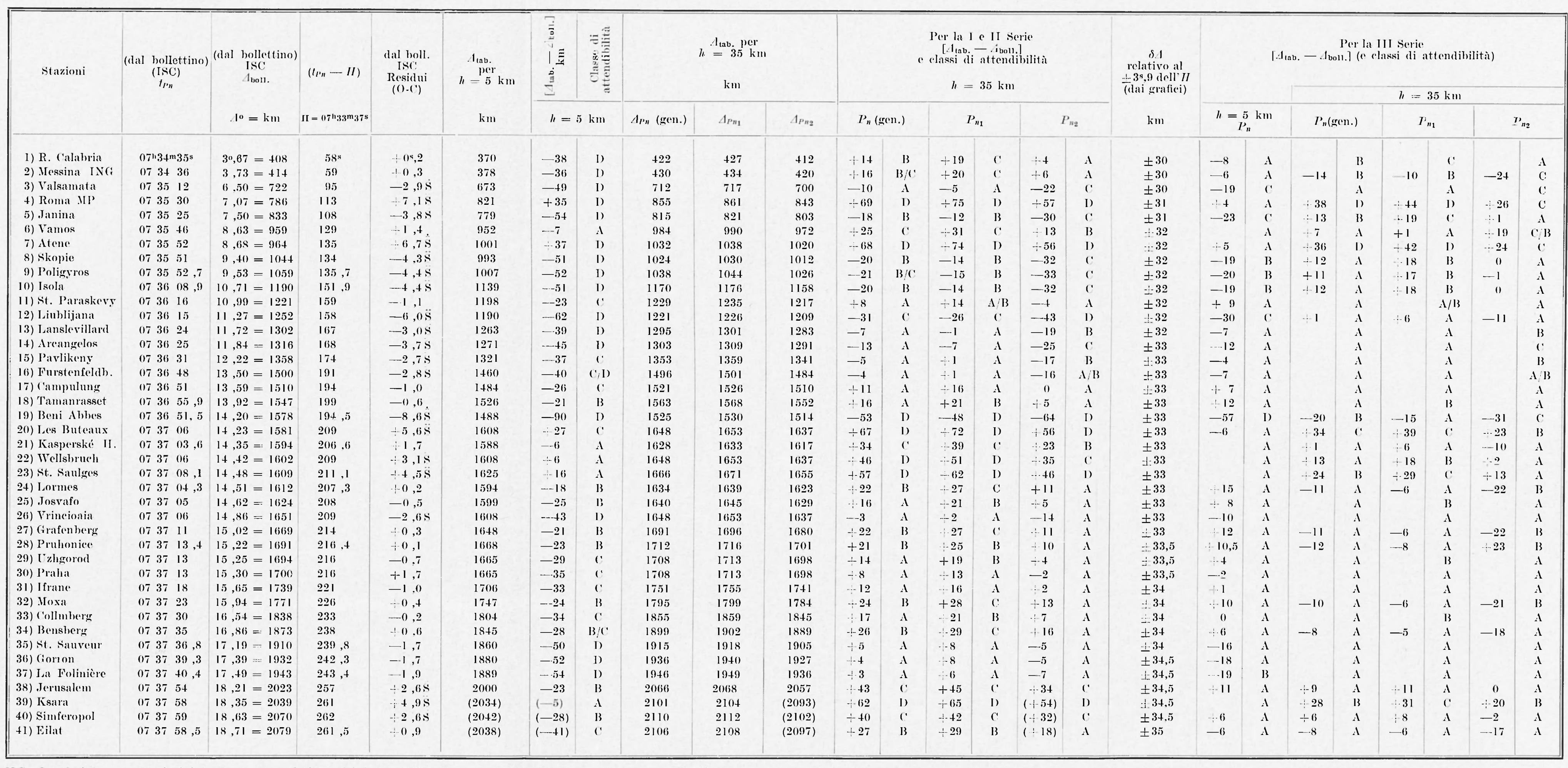

N.B. I valori tra parentesi si intendono estrapolati pereht: le rispective ilromocrone calcolite non aurivano a quecle distanze. 
$h=35 \mathrm{~km}$

(con 21 staz.)

\begin{tabular}{|l|l|l|}
\hline \multicolumn{1}{|c|}{$P_{n \text { (gen.) }}$} & \multicolumn{1}{|c|}{$P_{n_{1}}$} & \multicolumn{1}{|c|}{$P_{n_{2}}$} \\
\hline $\mathrm{A}=11 / 21=52,4 \%$ & $\mathrm{~A}=7 / 21=33,3 \%$ & $\mathrm{~A}=19 / 21=90,5 \%$ \\
$\mathrm{~B}=8 / 2 \mathrm{l}=38,1 \%$ & $\mathrm{~B}=6 / 21=28,6 \%$ & $\mathrm{~B}=2 / 21=9,5 \%$ \\
$\mathrm{C}=2 / 21=9,5 \%$ & $\mathrm{C}=8 / 21=38,1 \%$ & $\mathrm{C}=0$ \\
$\mathrm{D}=0$ & $\mathrm{D}=0$ & $\mathrm{D}=0$ \\
$(\mathrm{~A}+\mathrm{B})=90,5 \%$ & $(\mathrm{~A}+\mathrm{B})=61,9 \%$ & $(\mathrm{~A}+\mathrm{B}):=100 \%$ \\
\hline
\end{tabular}

Come si vede, la situazione delineata con la I serie permane convalidandosi. La $h=5 \mathrm{~km}$ resta insoddisfacente mentre per $h=35 \mathrm{~km}$ scompaiono tutte le classi $\mathrm{D}$ e, per le $P_{n 2}$, anche le classi C.

Le $P_{n 2}$ si presentano in questa serie con un $(A+B)-100 \%$. Per questo terremoto, che presenta un forte errore di $H( \pm 3$ s,9) abbiamo calcolato anche la III Serie. Questo errore comporta, per le distanze epicentrali, oscillazioni di $\pm(30-35) \mathrm{km}(\mathrm{v}$. fig. 3) per cui molte stazioni di classe C o D potrebbero trasferirsi nelle classi superiori. (Naturalmente questa serie va calcolata su tutte le stazioni considerate). I risultati ottenuti sono i seguenti:

III serie

$h=5 \mathrm{~km}$

(con 41 stazioni)

$P_{n}$
$\mathrm{~A}=33 / 41=80,5 \%$
$\mathrm{~B}=4 / 41=9,8 \%$
$\mathrm{C}=3 / 41=7,3 \%$
$\mathrm{D}=1 / 41=2,4 \%$

$h=35 \mathrm{~km}$

(con 41 staz.)

\begin{tabular}{|c|c|c|}
\hline$P_{n}$ (gen.) & $P_{n_{1}}$ & $P_{n_{2}}$ \\
\hline $\begin{array}{l}\mathrm{A}=32 / 41=78 \% \\
\mathrm{~B}=6 / 41=14,6 \% \\
\mathrm{C}=2 / 41=4,9 \% \\
\mathrm{D}=1 / 41=2,4 \% \\
(\mathrm{~A}+\mathrm{B})=92,6 \%\end{array}$ & $\begin{array}{l}\mathrm{A}=25 / 41=61 \% \\
\mathrm{~B}=9 / 41=22 \% \\
\mathrm{C}=5 / 41=12,2 \% \\
\mathrm{D}=2 / 41=4,9 \% \\
(A+\mathrm{B})=83 \%\end{array}$ & $\begin{array}{l}\mathrm{A}=26 / 41=63,4 \% \\
\mathrm{~B}=9 / 41=22,0 \% \\
\mathrm{C}=6 / 41=14,6 \% \\
\mathrm{D}=0 \\
(\mathrm{~A}+\mathrm{B})=85,4 \%\end{array}$ \\
\hline
\end{tabular}


Questa III seric ripropone l'attendibilita di $h=5 \mathrm{~km}$ pur continuando a mantenere valicli i risultati di $h=35 \mathrm{~km}$.

La $I V$ serie (ottenuta ritenendo che le $P_{n}$ siano o $P_{n 1}$ o $P_{n 2}$ ) applicabile solo alla $h=35 \mathrm{~km}$ (per $h=5 \mathrm{~km}$ non è distinzione tra le varie $P$ ).

I risultati sono i seguenti:

per $h=\check{5} \mathrm{~km}$ restano i risultati clelle serie precententi per $h=35 \mathrm{l} / \mathrm{m}$ si ha invece:

$$
\begin{aligned}
& \text { con tutte le } \quad\left\{\begin{array}{l}
A=25 / 41=61 \% \\
B=6 / 41=14,6 \%
\end{array}\right)(A+B)=70,6 \% \\
& +1 \text { stazioni } \mathrm{C}=4 / 41=9,8 \% \\
& \text { I) }=6 / 41=14,6 \% \\
& \hbar=3 \check{5} \mathrm{~km} \\
& \begin{array}{l}
\begin{array}{l}
A=19 / 21=90,5 \% \\
\text { con le 21 sta- }
\end{array} \\
\text { zioni sicure }
\end{array} \begin{array}{l}
\mathrm{C}=0 \\
1)=0
\end{array}
\end{aligned}
$$

Considerazion i gen erali. Malle analisi fatte risulterebbe una preferenza generale per la $h=35 \mathrm{~km}$ sia la II serie sia la IV conducono a ottimi risultati.

Le stazion i italiane che hamno registato sono Reggio C., Messina e Roma MP (notare che la Mag. è 4,4); i loro dati però si adatterebbero meglio alla dromocrona $h=5 \mathrm{~km}$; le prime onde registrate a Roma sono certamente onde successive alle $P_{n}$ essendo $(\mathrm{O}-\mathrm{C})=+7 \mathrm{~s}, 1$.

Come si verle, il procerlimento di analisi è piuttosto laborioso.

Per gli altri 24 terremoti esaminati ci limiteremo an esporne, in forma sintetica, gli elementi essenziali.

Terr. n. 1) Portogallo

$H=23^{n} 10^{\mathrm{m} \cdot 2 \mathrm{~s}}, 4 \pm 0 \mathrm{~s}, 35$

$\mathrm{SI})=1,41$ (su 21 osservazioni)

$h_{\mathrm{ISC}}=9 \mathrm{~km}$

$7_{\mathrm{MDD}}=20 \mathrm{~km}$

Magn: 4,6
Stazioni prese in esame fino a $20^{\circ}$ : $N_{1}=1 \stackrel{2}{2}$ di eui però $2(\mathrm{~S})$

Stazioni senza incertezze iniziali $N_{0}=10$

$N_{2} / N_{1}=83,3 \%$ (percent uale delle stazioni valide in partenza) 
Il controllo ì stato eseguito per le dromocrone relative alle seguenti profondita

$$
\left\{\begin{array}{l}
h_{1}=5 \mathrm{~km} \\
h_{2}=17,5 \mathrm{~km} \leftarrow
\end{array}\right.
$$

Osservazioni generali. Il terremoto è stato registrato da poche stazioni che coprono le $A$ in maniera piuttosto discontinua. Da notare che tra l' $H_{\text {(1BCIs) }}$ e l' $H_{\text {(ISc) }}$ ci sono 2 s,6 di diflerenza (v. tab. 1 ). I controlli vengono effettuati con le sole $P_{n}$ (le dromocrone per $h=5 \mathrm{~km}$ e $h=17,5 \mathrm{~km}$ hamno un solo tipo (li $P$ ).

Isa $I$ serie (con $N_{1}$ stazioni) dà i seguenti risultati:

$$
\begin{gathered}
h_{1}=5 \mathrm{~km} \\
\text { (con } 12 \text { stazioni) }
\end{gathered}\left\{\begin{array}{l}
A=8,3 \% \\
\mathrm{~B}=16,6 \% \\
\mathrm{C}=33,3 \% \\
\mathrm{D}=41,8 \% \\
\begin{array}{l}
\Lambda \\
h_{2}=66,6 \% \\
\text { (con } 12 \text { stazioni) }
\end{array} \\
\mathrm{B}=25 \% \\
\mathrm{C}=8,3 \% \\
\mathrm{D}=0
\end{array}\right.
$$

Prevale senz'altro $l^{\prime} h=1 \bar{r}, \tilde{5} \mathrm{~km}$. Fin d'ora mancano gli scarti più grandi $(\mathrm{I}=0 \%)$

La II serie (con $N_{2}$ stazioni) dà:

$$
\begin{aligned}
& \left.h_{1}=5 \mathrm{~km} \quad \begin{array}{l}
\Lambda=0 \\
B=20 \%
\end{array}\right\}(A+B)=20 \% \\
& (\operatorname{con} 10 \text { stazioni }) \mid \begin{array}{l}
\mathrm{O}=40 \% \\
\mathrm{D}=40 \%
\end{array} \\
& \left.h_{2}=17,5 \mathrm{~km} \quad \begin{array}{l}
A=80 \% \\
B=20 \%
\end{array}\right\}(A+B)=100 \% \\
& \text { (con } 10 \text { stazioni) }\left\{\begin{array}{l}
\mathrm{C}=0 \\
\mathrm{I}=0
\end{array}\right.
\end{aligned}
$$

$\left.{ }^{*}\right)$ La freceia sta ad inclicalre il risultato migliore (anche per i terremoti successivi). 
Questa serie conferma i risultati della I serie. $\mathrm{I} \%=5 \mathrm{bm}$ è da scartare senz'altro, mentre $1 \% h=17,5 \mathrm{~km}$ acquista una ottima credibilità.

Ia III serie non è necessario calcolarla perché la correzione di $H$ è piccola $( \pm 0$ s, 35$)$.

La $I V$ serie (le $P_{n}$ sono $P_{n 1}$ o $P_{n 2}$ ) non si può calcolare perché per entrambe le profondità consirlerate c'è un solo tipo ri $P_{n}$.

$\mathrm{R}$ is ultati finali. Il controllo con la dromocrona per $h=17,5 \mathrm{~km}$ dà risultati che sono da ritenersi senz'altro più validi.

Is e stazion i italian ne non hamo registrato, ad eccezione di Roma MP cui però giungono solo le $S_{n}$.

Terr. n. 2) ad W di Gibilterm

$H=18^{\mathrm{n}} 14^{\mathrm{m}} 25^{\mathrm{s}} \pm 1^{\mathrm{s}, 0}$

$\mathrm{SD}=2,12$ (su 23 osservazioni)

$h_{\mathrm{ISC}}=(42 \pm 11) \mathrm{km}$

$\hbar_{\text {USCGS }}=33 \mathrm{~km}$

Stazioni prese in esame fino a $20^{\circ}$ :

$x_{1}=12$ di cui $\begin{cases}2 & (\dot{\mathrm{S}}) \\ 2 & (\ddot{\mathrm{S}})\end{cases}$

Stazioni senza incertezze iniziali:

$$
N_{2}=8
$$

Magll: 4,2

$N_{2} / N_{1}=66,7 \%$ (percentuale delle stazioni valide in partenza)

Il controllo è stato eseguito per le clromocrone relative alle seguenti profonditid

$$
\left\{\begin{array}{l}
h_{1}=30 \mathrm{~km} \\
h_{2}=40 \mathrm{~km}
\end{array}\right.
$$

Os s e r vazion i gener a $1 \mathrm{i}$. Il terremoto è stato registrato la poche stazioni: le $\Delta$ fino a $20^{\circ}$ sono coperte in maniera rliscontinua (e'è un vuoto tra $8^{\circ}$ e $17^{\circ}$ ). I dati di partenza non sono troppo validi, specialmente l'H a confronto con i valori degli altri osservatori

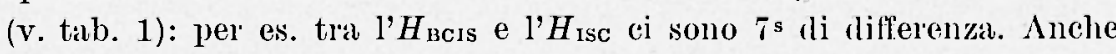
l'SI) denota gravi incertezze.

Tuttavia il nostro controllo si eftettua, per uniformiti, rispetto ai dati dell'ISC sopra trascritti. 
I. risultati sono i seguenti:

$I$ serie (con $N_{1}=12$ stazioni):

$$
h_{1}=30 \mathrm{~km}
$$

\begin{tabular}{|c|c|c|}
\hline$I_{n}$ (gen.) & $I_{n_{1}}$ & $I_{122}^{\prime}$ \\
\hline $\begin{array}{l}A=41,7 \% \\
B=25 \% \\
C=16,7 \% \\
D=16,7 \% \\
(A+B)=66,7 \%\end{array}$ & $\begin{array}{l}A=58,3 \% \\
B=8,3 \% \\
C=0 \% \\
D=33,3 \% \\
(A+B)=66,6 \%\end{array}$ & $\begin{array}{l}A=16,7 \% \\
B=8,3 \% \\
C=66,7 \\
D=8,3 \\
(A+B)=25 \%\end{array}$ \\
\hline
\end{tabular}

$$
h_{2}=40 \mathrm{~km}
$$

\begin{tabular}{|l|l|l|}
\hline \multicolumn{1}{|c|}{$P_{n}$ (gen.) } & \multicolumn{1}{|c|}{$P_{{ }_{11}}$} & \multicolumn{1}{c|}{$P_{n_{2}}$} \\
\hline & \multicolumn{1}{|c|}{} & \\
$\mathrm{B}=25 \%$ & $\mathrm{~A}=50 \%$ & $\mathrm{~A}=16,7 \%$ \\
$\mathrm{C}=16,7 \%$ & $\mathrm{~B}=8,3 \%$ & $\mathrm{~B}=25 \%$ \\
$\mathrm{D}=16,7 \%$ & $\mathrm{C}=25 \%$ & $\mathrm{C}=50 \%$ \\
$(\mathrm{~A}+\mathrm{B})=66,7 \%$ & $\mathrm{D}=16,7 \%$ & $\mathrm{D}=8,3 \%$ \\
& $(\mathrm{~A}+\mathrm{B})=58,3 \%$ & $(\mathrm{~A}+\mathrm{B})=+1,7 \%$ \\
\hline
\end{tabular}

Si delineano risultati migliori per $h_{1}=30 \mathrm{~km}$, specialmente rispetto alla $P_{n 1}$, però la somma delle percentuali $(\mathrm{I}+\mathrm{I})$ è ancora molto elevata. Si nota la forte incidenza delle 4 stazioni con notevoli (O-C) (v. tab. 2 resirlui).

II serie (con $N_{2}=8$ stazioni):

$$
h_{1}=30 \mathrm{~km}
$$

\begin{tabular}{|l|l|l|}
\hline \multicolumn{1}{|c|}{$P_{n}^{\prime}$ (gen.) } & \multicolumn{1}{|c|}{$P_{n_{1}}$} & \multicolumn{1}{c|}{$P_{n_{2}}$} \\
\hline & & \\
$\mathrm{A}=62,5 \%$ & $\mathrm{~A}=87,5 \%$ & $\mathrm{~A}=\mathbf{2 5 \%}$ \\
$\mathrm{B}=37,5 \%$ & $\mathrm{~B}=12,5 \%$ & $\mathrm{~B}=0$ \\
$\mathrm{C}=0$ & $\mathrm{C}=0$ & $\mathrm{C}=75 \%$ \\
$\mathrm{I}=0$ & $\mathrm{D}=0$ & $\mathrm{I}=0$ \\
$(\mathrm{~A}+\mathrm{B})=100 \%$ & $(\mathrm{~A} \div \mathrm{B})=100 \%$ & $(\mathrm{~A} \div \mathrm{B})=25 \%$ \\
\hline
\end{tabular}




$$
h_{2}=40 \mathrm{~km}
$$

\begin{tabular}{|l|l|l|}
\hline \multicolumn{1}{|c|}{$P_{n}$ (gen.) } & \multicolumn{1}{|c|}{$P_{n_{1}}$} & $P_{n_{2}}$ \\
\hline & & \\
$\mathrm{A}=37,5 \%$ & $\mathrm{~A}=75 \%$ & $\mathrm{~A}=25 \%$ \\
$\mathrm{C}=62,5 \%$ & $\mathrm{~B}=12,5 \%$ & $\mathrm{~B}=37,5 \%$ \\
$\mathrm{D}=0$ & $\mathrm{C}=12,5 \%$ & $\mathrm{C}=37,5 \%$ \\
$(\mathrm{~A}+\mathrm{B})=100 \%$ & $\mathrm{D}=0$ & $\mathrm{D}=0$ \\
& $(\mathrm{~A}+\mathrm{B})=87,5 \%$ & $(\mathrm{~A}+\mathrm{B})=62,5 \%$ \\
\hline
\end{tabular}

Resta confermato l'andamento della I serie: i risultati sono migliorati, specialmente per le $P_{n 1}\left(7_{h}=30 \mathrm{~km}\right)$; infatti il numero degli A raggiunge il valore dell' $87,5 \%$.

La III scrie non è stata calcolata: la correzione di $H\left( \pm 1^{\mathrm{s}}, 0\right)$ non comporterebbe grandi mutamenti.

La $I V$ serie (le $P_{n}$ sono o $P_{n 1}$ o $P_{n 2}$ ) dà i risultati seguenti:

$$
\begin{aligned}
& \left.h_{1}=30 \mathrm{~km} \mid \begin{array}{l}
\mathrm{A}=66,7 \% \\
\mathrm{~B}=8,3 \%
\end{array}\right\}(\mathrm{A}+\mathrm{B})=75 \% \quad h_{1}=30 \mathrm{~km} \mid \begin{array}{l}
\Lambda=100 \% \\
\mathrm{~B}=0
\end{array}
\end{aligned}
$$

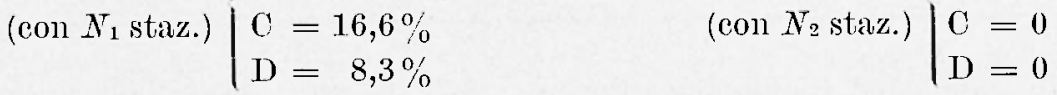

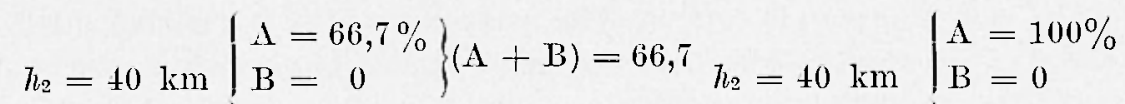

$$
\begin{aligned}
& \text { (con } \left.N_{1} \text { staz. }\right) \mid \begin{array}{ll}
\mathrm{C}=33,3 \% \\
\mathrm{D}=0
\end{array} \quad\left(\operatorname{con} N_{2} \text { staz. }\right) \mid \begin{array}{l}
\mathrm{C}=0 \\
\mathrm{D}=0
\end{array}
\end{aligned}
$$

Ris ultati finali. Questa IV serie porterebbe a ritenere attendibili entrambe le $h$, e questo confermerebbe le discordanze iniziali. Tuttavia l'esame generale delle varie serie, porta a dare una lieve preferenza alla $h=30 \mathrm{~km}$.

Qnesto, d'altra parte, concorderebbe anche con i $33 \mathrm{~km}$ dell'USCGS, con i $25 \mathrm{~km}$ dati dalla rete sismica spagnola ed anche con uno dei due valori possibili dell'ISO $h=(42 \pm 11) \mathrm{km}$.

Le stazioni italiane non hamno registrato il sisma, ma si noti che $M=4,2$. 
Terr. 11. 3) Marocco

$H=10^{\mathrm{h}} 03^{\mathrm{m}} 2 \cdot 2 \mathrm{~s} \pm 2 \mathrm{~s}, 6$

$\mathrm{SD}=1,66$ (su 30 osservazioni)

$h_{\text {Isc }}=(14 \pm 17) \mathrm{km}$

Magll: 4,5
Stazioni prese in esame fino a $20^{\circ}$. $N_{1}=10$ tutte senza incertezze iniziali

per cui $N_{2}=N_{1}=10$

$N_{2} / N_{1}=1.00 \%$ (percentuale rlelle stazioni valirle in partenza).

Il controllo è stato eseguito per le rlromocrone relative alle seguenti profonrlità:

$$
\left\{\begin{array}{l}
h_{1}=17,5 \mathrm{~km} \\
h_{2}=30 \mathrm{~km} \leftarrow
\end{array}\right.
$$

Osservazion i ge nerali. Le stazioni rlisponibili fino a $20^{\circ}$ sono poche, ma tutte valide in partenza: tuttavia esse non coprono con continuità l'intervallo rlelle $A$, poiché tra $8^{\circ}$ e $19^{\circ}$ e'è un vuoto consirlerevole.

Sia la $h$ che l'H sono rate con forti errori: è da prevedere ma incertezza nei risultati.

La $I$ serie e la $I I$ serie danno gli stessi risultati $\left(N_{2}=N_{1}-10\right)$ e ciò̀:

$$
\begin{aligned}
& \begin{array}{c|c}
h_{1}=17,5 \mathrm{~km} & \left.\begin{array}{l}
\mathrm{A}=40 \% \\
\mathrm{~B}=20 \% \\
\mathrm{C}=40 \% \\
\mathrm{D}=0 \%
\end{array}\right\}(\mathrm{A}+\mathrm{B})=60 \%
\end{array} \\
& h_{s}=30 \mathrm{~km}
\end{aligned}
$$

\begin{tabular}{|l|l|l|}
\hline \multicolumn{1}{|c|}{$P_{n}$ (gon.) } & \multicolumn{1}{|c|}{$P_{n_{1}}$} & \multicolumn{1}{c|}{$P_{n_{2}}$} \\
\hline & & \\
$\mathrm{B}=50 \%$ & $\mathrm{~A}=30 \%$ & $\mathrm{~A}=80 \%$ \\
$\mathrm{C}=10 \%$ & $\mathrm{~B}=20 \%$ & $\mathrm{~B}=20 \%$ \\
$\mathrm{D}=0$ & $\mathrm{C}=50 \%$ & $\mathrm{C}=0$ \\
$(\mathrm{~A}+\mathrm{B})=90 \%$ & $\mathrm{D}=0$ & $(\mathrm{~A} \div \mathrm{B})=100 \%$ \\
\hline
\end{tabular}

Queste serie damno una netta preferenza ai risultati ottenuti per $h=30 \mathrm{~km}$, per le onde $P_{n 2}$. 
La III serie (calcolata a causa della forte correzione di $H$ $( \pm 2 s, 6)$ dià:

$$
h_{1}-17,5 \mathrm{~km}\left\{\begin{array}{l}
A=80 \% \\
B=20 \% \\
C=0 \\
D=0
\end{array}\right)(A+B)=1.00 \%
$$

$h_{2}=30 \mathrm{~km}$

\begin{tabular}{|l|l|l|}
\hline \multicolumn{1}{|c|}{$P_{n \text { (gen.) }}$} & \multicolumn{1}{|c|}{$P_{n_{1}}$} & \multicolumn{1}{c|}{$P_{u_{2}}$} \\
$\mathrm{~A}=90 \%$ & $\mathrm{~A}=100 \%$ & $\mathrm{~A}=\mathbf{3 0 \%}$ \\
$\mathrm{B}=10 \%$ & $\mathrm{~B}=0$ & $\mathrm{~B}=\mathbf{3 0 \%}$ \\
$\mathrm{C}=0$ & $\mathrm{C}=0$ & $\mathrm{C}=40 \%$ \\
$\mathrm{D}=0$ & $\mathrm{D}=0$ & $\mathrm{I}=0$ \\
$(\mathrm{~A}+\mathrm{B})=100 \%$ & $(\mathrm{~A}+\mathrm{B})=100 \%$ & $(\mathrm{~A}+\mathrm{B})=60 \%$ \\
\hline
\end{tabular}

I risultati di questa serie sono ottimi per entrambe le $h$. Una lieve preferenza spetterebbe alle $P_{n 1}$ per $\hbar=30 \mathrm{~km}$.

La $I V$ serie (le $P_{n}$ sono o $P_{n 1}$ o $P_{n 2}$ ) conferma ovviamente questa preferenza, con

$$
\Lambda=1.00 \%
$$

$R$ is u ltati finali. Il controllo eseguito farebbe scegliere $1 \%=30 \mathrm{~km}$, però non esclude una profonditì minore.

Le stazioni italiane non hanno registrato.

Terr. n. 4) Algeria

$H=00^{\mathrm{n}} 31^{\mathrm{m}} 49^{\mathrm{s}} \pm 1^{\mathrm{s}, 5}$

$\mathrm{SD}=2,52($ su 35 osservazioni)

$h_{1 \mathrm{sc}}=(32 \pm 16) \mathrm{km}$

huscas $=36 \mathrm{~km}$

Magr: 4,6
Stazioni prese in esame fino a $20^{\circ}$ :

$$
\begin{array}{l|lc}
N_{1}=19 & (\dot{\mathrm{S}}) \\
3 & (\ddot{\mathrm{S}}) \\
1 & (\dot{\mathrm{S}})
\end{array}
$$

Stazioni senza incertezze iniziali $\Lambda_{2}=12$

$N_{2} / N_{1}=63 \%$ (percentuale delle stazioni valide in partenza) 
Il controllo è stato eseguito per le dromocrone relative alla seguente profonclitì:

$$
h=35 \mathrm{~km} \leftarrow
$$

Osservazioni generali. Le stazioni coprono con contimuita le $\Delta$ fino a $20^{\circ}$.

L'SD è piuttosto elevato, ma le $h$ sono abbastanza concordi per' eui il controllo è fatto per una sola profondità: $h=35 \mathrm{~km}$.

La $I$ serie (con $N_{1}=19$ stazioni) dà:

$$
h=35 \mathrm{~km}
$$

\begin{tabular}{|c|c|c|}
\hline$P_{n}$ (gen.) & $P_{n_{1}}$ & $P_{n_{2}}$ \\
\hline $\begin{array}{l}A=58 \% \\
B=10,5 \% \\
C=10,5 \% \\
D=21 \% \\
(A+B)=68,5 \%\end{array}$ & $\begin{array}{l}\mathrm{A}=\mathbf{5 2 , 6 \%} \\
\mathrm{B}=15,8 \% \\
\mathrm{C}=15,8 \% \\
\mathrm{D}-15,8 \% \\
(\mathrm{~A}+\mathrm{B})=\mathbf{6 8 , 4 \%}\end{array}$ & $\begin{array}{l}A=47,5 \% \\
B=15,8 \% \\
C=21 \% \\
D=15,8 \% \\
(A+B)=63,3 \%\end{array}$ \\
\hline
\end{tabular}

N.B. Nelle $P_{n}$, trovano posto come $A$ due stazioni contrassegnate in partenza con $\dot{S}$ (Ebro e la Folinière) (v. tab. residui).

La II serie (con $\bar{N}_{2}=12$ stazioni) dà:

$$
h=35 \mathrm{~km}
$$

\begin{tabular}{|l|l|l|}
\hline \multicolumn{1}{|c|}{$P_{n}$ (gen.) } & \multicolumn{1}{|c|}{$P_{n_{1}}$} & \multicolumn{1}{c|}{$P_{n_{2}}$} \\
\hline $\mathrm{A}=91,6 \%$ & $\mathrm{~A}=83,4 \%$ & $\mathrm{~A}=58,5 \%$ \\
$\mathrm{~B}=8,4 \%$ & $\mathrm{~B}=16,6 \%$ & $\mathrm{~B}=25 \%$ \\
$\mathrm{C}=0$ & $\mathrm{C}=0$ & $\mathrm{C}=1(\mathbf{6}, 6 \%$ \\
$\mathrm{D}=0$ & $\mathrm{D}=0$ & $\mathrm{D}=0$ \\
$(\mathrm{~A}+\mathrm{B})=100 \%$ & $(\mathrm{~A}+\mathrm{B})=100 \%$ & $(\mathrm{~A}-\mathrm{B})=83,5 \%$ \\
\hline
\end{tabular}

anche qui, nella II serie, scompaiono i D e quasi tutti i C.

La $I I I$ serie non viene calcolata perché l'errore di $H$ è abbastanza piccolo $( \pm 1 \mathrm{~s}, 5)$, e quindi non può offrire sensibili variazioni rispetto alle precerlenti. 
La $I V$ serie invece (le $P_{n}$ sono o $P_{n 1}$ o $P_{n 2}$ ) porta a sensibili miglioramenti: essal da:

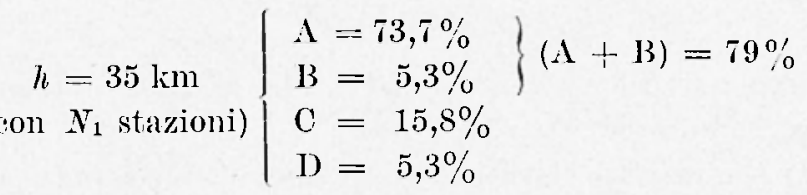

$$
\begin{aligned}
& \left.\begin{array}{c|l}
h-35 \mathrm{~km} & \begin{array}{l}
\mathrm{A}=100 \% \\
\mathrm{~B}=0 \\
\mathrm{C}=0 \\
\mathrm{D}=0
\end{array}
\end{array}\right\}(\mathrm{A}+\mathrm{B})=100 \%
\end{aligned}
$$

Per tutte le stazioni (valide in partenza) le prime onde sono o $P_{n 1}$ o $P_{n 2}$.

Risultati finali. Il controllo fatto con $h=35 \mathrm{~km}$ è da ritenersi molto sodrlisfacente sia per i risultati cui porta, sia perchó sembra convalidare l'esistenza delle $P_{n_{2}}$ come prime onde rilevate la molte stazioni.

Le stazion i taliane non hamno registrato il sisma $(M=4,6)$ : solo Roma riporta una fase successiva alle $P_{n}$.

Ter'r. 11. 5) Algeria

$$
\begin{aligned}
& H=00^{n} 27^{m 05}, \tau \pm 0 \text { s }, 50 \\
& \mathrm{SD}=1,70 \text { (su } 20 \text { osservazioni) } \\
& h_{\text {Isc }}=17 \mathrm{~km} \\
& h_{\text {rete spagnola }}=33 \mathrm{~km} \\
& N_{1}=1+\quad \text { I2 (S) } \\
& \text { di eui però / } 1 \text { (̈) } \\
& \text { Stazioni senza incertezze iniziali. } \\
& N_{2}=1.1 \\
& N_{2}{ }^{\prime} N_{1}=78,6 \% \text { (percentuale relle } \\
& \text { stazioni valirle in partenza) }
\end{aligned}
$$

Il controllo è stato eseguito per le dromocrone relative alle seguenti profonditic

$$
\left\{\begin{array}{l}
7_{1}=17,5 \mathrm{~km} \\
h_{2}=35 \mathrm{~km} \leftarrow
\end{array}\right.
$$

Osservazion i generali. Il terremoto ha dati di partenza abbastanza buoni. I'errore di $H$ è piccolo, quello di $h$ non è dato, tuttavia tenuto presente il valore di $33 \mathrm{~km}$ riportato clalle sta- 
zioni della rete spagnola abbiamo effettuato il controllo per due valori di $h$.

Il terremoto (entro i $20^{\circ}$ ) è stato registrato con ma certa continuitì fino ai $14^{\circ}$ di Tamanrasset; dopo si incontra solo Josvafo a $\sim 18^{\circ}$.

Isa $I$ Serie dà i seguenti risultati: (con $N_{1}=14$ stazioni)

$$
h_{1}=17,5 \mathrm{~km}\left\{\begin{array}{c}
\text { solo } P_{n} \\
\Lambda=64,2 \% \\
\mathrm{~B}=14,3 \% \\
\mathrm{C}=7,2 \% \\
\mathrm{D}=14,3 \%
\end{array}\right\}(\mathrm{A}+\mathrm{B})=78,5 \%
$$

$h_{2}=35 \mathrm{~km}$

\begin{tabular}{|l|l|l|}
\hline \multicolumn{1}{|c|}{$P_{n}$ (gen.) } & \multicolumn{1}{|c|}{$P_{n_{1}}$} & \multicolumn{1}{c|}{$P_{n_{2}}$} \\
\hline & & \\
$\mathrm{A}=71,5 \%$ & $\mathrm{~A}=71,5 \%$ & $\mathrm{~A}=57 \%$ \\
$\mathrm{~B}=0$ & $\mathrm{~B}=7,1 \%$ & $\mathrm{~B}=21,5 \%$ \\
$\mathrm{C}=21,5 \%$ & $\mathrm{C}=14,3 \%$ & $\mathrm{C}=7,1 \%$ \\
$\mathrm{D}=7,1 \%$ & $\mathrm{D}=7,1 \%$ & $\mathrm{D}=14,3 \%$ \\
$(\mathrm{~A}+\mathrm{B})=71,5 \%$ & $(\mathrm{~A}+\mathrm{B})=78,6 \%$ & $(\mathrm{~A}+\mathrm{B})=78,5 \%$ \\
\hline
\end{tabular}

Migliori risultati si hanno per $h_{2}=35 \mathrm{~km}$.

La $I I$ serie (con $N_{2}=11$ stazioni) dà:

$$
\begin{gathered}
\text { solo } P_{n} \\
h_{i}=1 ., 5 \mathrm{~km} \\
\begin{array}{l}
A=73 \% \\
\mathrm{~B}=18 \% \\
\mathrm{C}=9 \% \\
\mathrm{D}=0
\end{array}
\end{gathered}
$$

$$
h_{,}=35 \mathrm{~km}
$$

\begin{tabular}{|l|l|l|}
\hline \multicolumn{1}{|c|}{$P_{n}$ (gen.) } & \multicolumn{1}{|c|}{$P_{n_{1}}$} & \multicolumn{1}{c|}{$P_{{ }_{n}}$} \\
\hline $\mathrm{A}=91 \%$ & $\mathrm{~A}=91 \%$ & $\mathrm{~A}=73 \%$ \\
$\mathrm{~B}=0$ & $\mathrm{~B}=0$ & $\mathrm{~B}=18 \%$ \\
$\mathrm{C}=9 \%$ & $\mathrm{C}=9 \%$ & $\mathrm{C}=9 \%$ \\
$\mathrm{D}=0$ & $\mathrm{D}=0$ & $\mathrm{I}=0$ \\
$(\mathrm{~A}+\mathrm{B})=91 \%$ & $(\mathrm{~A}+\mathrm{B})=91 \%$ & $(\mathrm{~A}+\mathrm{B})=91 \%$ \\
\hline
\end{tabular}


Ancora i migliori risultati si hamno per $h_{2}=35 \mathrm{~km}$, con lieve differenza rispetto ad $h_{1}=17,5 \mathrm{~km}$.

Ja $I I I$ serie non si calcola perché la correzione di $H$ è solo \pm 0 s,50.

Ja $I V$ serie (le $P_{n}$ sono $0 F_{n 1}$ o $P_{n 2}$ ) si può calcolare solo per $h_{2}=35 \mathrm{~km}$ : per $h_{1}=17,5$ si possono confermare qui i risultati della I e II serie.

$$
\begin{aligned}
& \left.h_{2}=35 \mathrm{~km} \quad \begin{array}{l}
A=71,4 \% \\
B=21,4 \%
\end{array}\right\}(A+B)=92,8 \% \\
& \left(\operatorname{con} N_{1} \text { stazioni) } \mid \begin{array}{l}
\mathrm{C}=0 \\
\mathrm{D}=7,1 \%
\end{array}\right. \\
& \left.h_{2}=35 \mathrm{~km} \quad \begin{array}{l}
A=91 \% \\
\mathrm{~B}=9 \%
\end{array}\right\}(A+B)=100 \% \\
& \text { (con } N_{2} \text { stazioni) } \mathrm{C}=0 \\
& \mathrm{D}=\mathbf{0}
\end{aligned}
$$

R is ultati finali. Si può dare una leggera preferenza ai risultati oftenuti per $\hbar=3 \check{\mathrm{km}}$.

Je stazioni italiane non hammo registrato il terremoto.

Terr. n. 6) Tunisia

$$
\begin{aligned}
& H=13^{\mathrm{h}} 40^{\mathrm{m}} 14^{\mathrm{s}} \pm 2 \mathrm{~s}, 0 \\
& \text { S.D }=3,83(\mathrm{su} 52 \text { osservazioni) } \\
& h_{\mathrm{rSc}}=(37 \pm 22) \mathrm{km}
\end{aligned}
$$

Magn: 4,8

Stazioni prese in esame fino a $20^{\circ}$.

$$
\begin{array}{l|lc}
N_{1}=30 & (\dot{\mathrm{S}}) \\
\text { di eui però } & 5 & (\ddot{\mathrm{S}}) \\
3 & (\dot{\mathrm{S}})
\end{array}
$$

Stazioni senza incertezze iniziali:

$$
N_{2}=13
$$

$N_{2} / N_{1}=43,3 \%$ (percentuale delle stazioni valide in partenza)

Il controllo is stato eseguito per la dromocrona relativa alla seguente profoncliti

$$
\eta=35 \mathrm{~km} \leftarrow
$$

Osservazioni generali. Il terremoto è stato registrato da numerose stazioni, che coprono le $\Delta$ fino a $20^{\circ}$ in maniera abbastanza continua: però meno della meta hamno residui accettabili (v. tab. 2 (O-C)). Il grancle disaccordo tra gli $H$ dati dai vari osservatori 
(disaceordo che arriva fino an un massimo di $1 . t$ sec. - v. tab. 1) griustifica il gran numero delle stazioni incerte fin dall"inizio. Soterole anche l'erore dell'h $( \pm 20 \mathrm{~km})$ e, naturalmente, l'errore standaud (SD).

Sono da prevedere risultati poco soddisfacenti.

Date le gravi incertezze iniziali, il controllo i stato fatto sulla sola profondità $h=35 \mathrm{~km}$.

La I serie (con $N_{1}$ stazioni) dà i seguenti risultati:

$$
\begin{gathered}
h=35 \mathrm{~km} \\
(\cdot 001 \mathrm{30} \text { stazioni) }
\end{gathered}
$$

\begin{tabular}{|c|c|c|}
\hline$P_{n}($ gen.) & $P_{n_{\mathrm{I}}}$ & $i_{n 2}$ \\
\hline $\begin{array}{l}\Lambda=26,7 \% \\
\mathrm{~B}=23,3 \% \\
\mathrm{C}=23,3 \% \\
1)=26,7 \% \\
(A+B)=50 \%\end{array}$ & $\begin{array}{l}A=23.3 \% \\
B=33,3 \% \\
C=20 \% \\
J)=23,3 \% \\
(A+B)=56,6 \%\end{array}$ & $\begin{array}{l}A=30 \% \\
B=6,7 \% \\
C=23,3 \% \\
D=40 \% \\
(A+B)=36,7 \%\end{array}$ \\
\hline
\end{tabular}

Ia $I I$ serie (con $N_{2}$ stazioni) da ovviamente risultati migliori:

$$
h=35 \mathrm{~km}
$$

(con 13 stazioni)

\begin{tabular}{|l|l|l|}
\hline \multicolumn{1}{|c|}{$P_{n}$ (gen.) } & \multicolumn{1}{|c|}{$P_{n_{1}}$} & $P_{n_{2}}$ \\
$\mathrm{~A}=61,5 \%$ & $\mathrm{~A}=53,8 \%$ & $\mathrm{~A}=46,2 \%$ \\
$\mathrm{~B}=30,8 \%$ & $\mathrm{~B}=46,2 \%$ & $\mathrm{~B}=15,4 \%$ \\
$\mathrm{C}=7,7 \%$ & $\mathrm{C}=0$ & $\mathrm{C}=30,8 \%$ \\
$\mathrm{D}=0$ & $\mathrm{D}=0$ & $\mathrm{D}=7,7 \%$ \\
$(\mathrm{~A}+\mathrm{B})=92,3 \%$ & $(\mathrm{~A}+\mathrm{B})=100 \%$ & $(\mathrm{~A} \div \mathrm{B})=61,6 \%$ \\
\hline
\end{tabular}

La III serie (calcolata su tutte le stazioni, tenendo conto della sensibile correzione dell $\left.H\left( \pm^{2}\right)^{*}\right)$ dà $\mathrm{i}$ seguenti risultati: 
$h=35 \mathrm{~km}$

(ron tutte le starioni)

\begin{tabular}{|l|l|l|}
\hline \multicolumn{1}{|c|}{$P_{n}$ (gen.) } & \multicolumn{1}{|c|}{$P_{n_{1}}$} & \multicolumn{1}{c|}{$P_{n_{2}}$} \\
$\mathrm{~A}=60 \%$ & $\mathrm{~A}=63,3 \%$ & $\mathrm{~A}=40 \%$ \\
$\mathrm{~B}=20 \%$ & $\mathrm{~B}=16,7 \%$ & $\mathrm{~B}=23,3 \%$ \\
$\mathrm{C}=6,7 \%$ & $\mathrm{C}=6,7 \%$ & $\mathrm{C}=30 \%$ \\
$\mathrm{D}=13,3 \%$ & $\mathrm{D}=13,3 \%$ & $6,7 \%$ \\
$(\mathrm{~A}+\mathrm{B})=80 \%$ & $(\mathrm{~A}+\mathrm{B})=80 \%$ & $(\mathrm{~A}+\mathrm{B})=63,3 \%$ \\
\hline
\end{tabular}

Questa III serie rispetto alla I costituisce un netto miglioramento su tutte e tre le $P$.

ì da notare il permanere di valori $\mathrm{C}$ e $\mathrm{D}$, ma questo era da attendersi giacché le stazioni $\dot{\mathrm{S}}, \ddot{\mathrm{S}}$ en $\dot{\hat{\mathrm{S}}}$ sono numerose, en è da ritenere che per quanto le prime onde siano state considerate come $P$ dall'ISC, siano in realta onde diverse, specialmente quelle relative alle $\stackrel{\mathrm{S}}{\mathrm{S}}$

I a IV serie (calcolata tenendo conto che le $P_{n}$ possono essere o $P_{n 1}$ o $\left.P_{n 2}\right)$ dà:

$$
\begin{gathered}
\qquad \begin{array}{c}
\text { (con tutte le } 30 \text { stazioni) } \\
\mathrm{B}=23,3 \% \\
\mathrm{C}=23,3 \% \\
\mathrm{D}=16,7 \%
\end{array} \\
\begin{array}{c}
\mathrm{A}=35 \mathrm{~km} \\
\text { (con le } 13 \text { stazioni valide) }
\end{array} \\
\begin{array}{l}
\mathrm{A}=61,5 \% \\
\mathrm{~B}=38,5 \% \\
\mathrm{C}=0 \\
\mathrm{D}=0
\end{array}
\end{gathered}
$$

$R$ is ultati fin a $1 \mathrm{i}$. L'analisi conferma le previsioni. Tue 4 serie calcolate costituiscono progressivi miglioramenti, ma rivelano sempre le gravi incertezze iniziali.

Ise stazioni italiane che hamno registrato sono Messina ING e Roma MP, però per entrambe, le $P$ arrivano in anticipo (v. tab. resirlui). 
Terr. 11. т) Tunisia

$H=01^{\mathrm{n}} 02^{\mathrm{m}} \mathrm{7}^{\mathrm{s}}, 9 \pm 0 \mathrm{~s}, 64$

$\mathrm{SD}=1,79$ (su 130 osservazioni)

$h_{\mathrm{ISC}}=(57 \pm 5,9) \mathrm{km}$

$h_{\mathrm{USCGS}}=24 \mathrm{~km}$

Magn: $\check{5} \sim$

Stazioni prese in esame fino a $20^{\circ}$.

$$
\begin{array}{c|c|cc}
N_{1}=43 & \text { di cui } & 7(\dot{\mathrm{S}}) \\
\left(h_{1}=30 \mathrm{~km}\right) & \text { però } & \ddot{(\mathbf{S}}) \\
N_{1}=42 & & 1(\dot{\mathrm{S}}) \\
\left(h_{2}=60 \mathrm{~km}\right) & & 1
\end{array}
$$

Stazioni senza incertezze iniziali:

$$
\begin{aligned}
& N_{2}=33\left(\text { per } h_{1}=30 \mathrm{~km}\right) \\
& N_{2}=32\left(\text { per } h_{2}=60 \mathrm{~km}\right)
\end{aligned}
$$

$$
N_{2}, N_{1}=\left(76,7 \%\left(\text { per } h_{1}=30 \mathrm{~km}\right)\right.
$$$$
\left(76,2 \% \text { (per } h_{2}=60 \mathrm{~km}\right)
$$

percentuali delle stazioni valide in partenza

Il controllo è stato eseguito per le dromocrone relative alle segruenti profondità

$$
\left\{\begin{array}{l}
h_{1}=30 \mathrm{~km} \\
h_{2}=60 \mathrm{~km} \leftarrow
\end{array}\right.
$$

Osservazioni gen erali. Gli elementi di partenza sono tutti abbastanza buoni: anche i resirlui (O-C) sono accettabili (v. tabella 2) e sia l'errore standard che l'errore dell' $( \pm \tilde{5}, 9 \mathrm{~km})$ rendono attendibili i dati di partenza. C'è da notare tuttavia un certo disaccordo tra i vari $H$ dati da altri osservatori: il massimo divario si ha con Mosea $\left(\Delta H_{\max }=|6 \mathrm{~s}, 9|\right)$.

Il controllo è stato fatto per le due profondità indicate, perché abbiamo tenuto conto di $h=24 \mathrm{~km}$ riportato rall'USCGS. [Si ricorda che la dromocrona per $h=60 \mathrm{~km}$ non si estende oltre $\left.A=17^{\circ}\left({ }^{3}\right)\right]$.

Le stazioni coprono con continuità l'intervallo delle $\Delta$ fino a $20^{\circ}$.

La I serie dà i seguenti risultati:

$$
h_{1}=30 \mathrm{~km}
$$

(con 43 stazioni)

\begin{tabular}{|l|l|l|}
\hline \multicolumn{1}{|c|}{$P_{n}$ (gen.) } & \multicolumn{1}{|c|}{$P_{n_{1}}$} & \multicolumn{1}{c|}{$P_{n_{2}}$} \\
\hline & & \\
$\mathrm{A}=58,1 \%$ & $\mathrm{~N}=60,5 \%$ & $\mathrm{~A}=34,9 \%$ \\
$\mathrm{~B}=25,6 \%$ & $\mathrm{~B}=20,9 \%$ & $\mathrm{~B}=25,6 \%$ \\
$\mathrm{C}=11,6 \%$ & $\mathrm{C}=11,6 \%$ & $\mathrm{O}=30,2 \%$ \\
$\mathrm{D}=4,7 \%$ & $\mathrm{O}=7,0 \%$ & $\mathrm{D}-\mathrm{9.3 \%}$ \\
$(\mathrm{A}+\mathrm{B})=83,7 \%$ & $(\mathrm{~A}+\mathrm{B})=81,4 \%$ & $(\mathrm{~A}+\mathrm{B})=60,5 \%$ \\
\hline
\end{tabular}




$$
h_{2}=60 \mathrm{~km}
$$

(con +2 stazioni)

\begin{tabular}{|c|c|c|}
\hline$I_{n}^{\prime}(g e n)$. & $P_{n_{1}}$ & $P_{n_{2}}$ \\
\hline $\begin{array}{l}A=69 \% \\
B=9,5 \% \\
C=16,7 \% \\
I=4,8 \% \\
(A+B)=78,5 \%\end{array}$ & $\begin{array}{l}A=61,9 \% \\
B=14,3 \% \\
C=19 \% \\
D=4,8 \% \\
(A+B)=76,2 \%\end{array}$ & $\begin{array}{l}A=45,2 \% \\
B=33,3 \% \\
C=16,7 \% \\
D=4,8 \% \\
(A+B)=78,5 \%\end{array}$ \\
\hline
\end{tabular}

Le due $h$ sembrano entrambe egualmente probabili (con una lieve preferenza per $h=60 \mathrm{~km}$ ).

Là II serie dà:

$$
h_{1}=30 \mathrm{~km}
$$

(con 33 starioni)

\begin{tabular}{|l|l|l|}
\hline \multicolumn{1}{|c|}{$P_{n}$ (ren.) } & \multicolumn{1}{|c|}{$P_{n_{1}}$} & $P_{n 9}$ \\
$\mathrm{~A}=72,7 \%$ & $\mathrm{~A}=78,8 \%$ & $\mathrm{~A}=33,3 \%$ \\
$\mathrm{~B}=24,2 \% \%$ & $\mathrm{~B}=21,2 \%$ & $\mathrm{~B}=30,3 \%$ \\
$\mathrm{C}=3,0 \% \%$ & $\mathrm{C}=0$ & $\mathrm{C}=36,4 \%$ \\
$\mathrm{D}=0$ & $\mathrm{D}=0$ & $\mathrm{I}=0$ \\
$(\mathrm{~A}+\mathrm{B})=97 \% \%$ & $(\mathrm{~A}+\mathrm{B})=100 \%$ & $(\mathrm{~A}+\mathrm{B})=63,6 \%$ \\
\hline
\end{tabular}

$$
h_{2}=60 \mathrm{~km}
$$

(con 32 stazioni)

\begin{tabular}{|l|l|l|}
\hline \multicolumn{1}{|c|}{$P_{n \text { (gen.) }}$} & \multicolumn{1}{|c|}{$P_{n_{1}}$} & \multicolumn{1}{c|}{$P_{n_{2}}$} \\
\hline & & \\
$\mathrm{A}=90,6 \%$ & $\mathrm{~A}=78,1 \%$ & $\mathrm{~A}=56,3 \%$ \\
$\mathrm{~B}=6,3 \%$ & $\mathrm{~B}=15,6 \%$ & $\mathrm{~B}=31,3 \%$ \\
$\mathrm{C}=3,1 \%$ & $\mathrm{C}=6,3 \%$ & $\mathrm{C}=12,5 \%$ \\
$\mathrm{I}=0$ & $\mathrm{D}=0$ & $\mathrm{I}=0$ \\
$(\mathrm{~A}+\mathrm{B})=97 \%$ & $(\mathrm{~A} \div \mathrm{B})=93,7 \%$ & $(\mathrm{~A}+\mathrm{B})=87,6 \%$ \\
\hline
\end{tabular}

Come al solito, i risultati di questa serie miglionano i precenlenti, confermando l'attendibilità di entrambe le $h$.

La $I I I$ serie non è stata calcolata perché l'errore di $H$ è piceolo

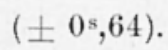


Lat $I V$ serie (le $P_{n}$ sono o $P n_{1}$ o $P_{n 2}$ ) dia:

$$
\begin{aligned}
& \left.h_{1}=30 \mathrm{~km} \quad \begin{array}{l}
A=83,7 \% \\
\mathrm{~B}=9,3 \%
\end{array}\right\}(A+B)=93 \% \\
& \text { (con } 43 \text { stazioni) } \mid \begin{array}{l}
\mathrm{C}=4,6 \% \\
\mathrm{D}=2,3 \%
\end{array} \\
& \left.h_{2}=60 \mathrm{~km} \quad \begin{array}{l}
\mathrm{A}=81 \% \\
\mathrm{~B}=11,9 \%
\end{array}\right\}(\mathrm{A}+\mathrm{B})=93 \% \\
& \text { (con }+2 \text { stazioni) } \mathrm{C}=2,4 \% \\
& \mathrm{I}=4,8 \% \\
& \left.h_{1}=30 \mathrm{~km} \quad \begin{array}{l}
\mathrm{A}=93,9 \% \\
\mathrm{~B}=6.1 \%
\end{array}\right\}(\mathrm{A}+\mathrm{B})=100 \% \\
& \text { (con } 33 \text { stazioni) } \mid \begin{array}{l}
\mathrm{C}=0 \\
\mathrm{D}=0
\end{array} \\
& \left.h_{2}=60 \mathrm{~km} \quad \begin{array}{l}
\mathrm{A}=100 \% \\
\mathrm{~B}=0
\end{array}\right\}(\mathrm{A}+\mathrm{B})=100 \% \\
& \text { (con } 32 \text { stazioni) } \mid \begin{array}{l}
\mathrm{C}=0 \\
\mathrm{D}=0
\end{array}
\end{aligned}
$$

In quest'ultima serie, pur permanendo la eguale probabiliti per le due $h$, si raggiunge il massimo delle $A$ (il $100 \%$ ) per $h=60 \mathrm{~km}$.

$R$ is ultati finali. Dalle analisi fatte si cleduce che pur essendo le due $h$ entrambe attendibili, ma lieve preferenza può andare ad $h_{2}=60 \mathrm{~km}$.

St a zi o n i it a li an e : hamo registrato Roma MP con un ottimo valore per la $P_{n 1}(h=60 \mathrm{~km})$ e Reggio Calabria il cui inizio non è certamente una $P$ : risulta classificata in $\mathrm{D}$ in entrambe le $\hbar$ ed infatti il suo residuo è $(\mathrm{O}-\mathrm{C})=+5 \mathrm{~s}, 5$ (v. tab. residui).

Terr. n. 8) Sicilia occidentale

$$
\begin{aligned}
& H=16^{\mathrm{m}} 266^{\mathrm{m} 0} 04^{\mathrm{s}} \pm 2 \mathrm{~s}, 0 \\
& \mathrm{SID}=2,17(\mathrm{su} 50 \text { osservazioni }) \\
& h_{\mathrm{sc}}=(27 \pm 15) \mathrm{km} \\
& h_{\mathrm{LSCGS}}=33 \mathrm{~km}
\end{aligned}
$$

Magn: $4,5-4,4$
Stazioni prese in esame fino a $20^{\circ}$.

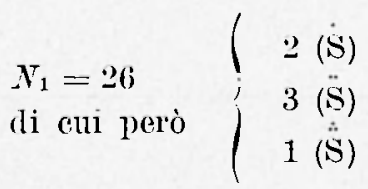

Stazioni senza incertezze iniziali:

$$
N_{2}=20
$$

$N_{2} / N_{1}=75 \%$ (percentuale delle stazioni valide in partenza) 
Il controllo è stato eseguito per la dromocrona relativa alla seguente profondità

$$
h=30 \mathrm{~km} \leftarrow
$$

Ossorvazion i generali. Questo terremoto appartiene al periodo sismico della Sicilia occidentale 1968 da noi studiato, peró non si trova tra quelli inclusi in letto studio, sicché la sua analisi può servire ottimamente come controllo dei risultati ottenuti e della tecnica usata nella presente inclagine.

Notiamo che il valore di $h=(27 \pm 15) \mathrm{km}$ dato dall'ISC ripropone l'incertezza da noi messa in evidenza fin clalla prima nota. (*) a proposito della profondità ipocentrale, incertezza successivamente eliminata per mezzo di calcoli accurati che hanno consentito per ogni terremoto di indicarne la profondità.

Per mantenerci su un valore medio, tenuto anche presente $h_{\text {Uscas }}=33 \mathrm{~km}$, abbiamo fatto il controllo per $h=30 \mathrm{~km}$.

Le stazioni prese in esame (fino a $20^{\circ}$ ) coprono le $\Delta$ con continuità solo fino a $15^{\circ}$ : il rimanente intervallo è scarsamente coperto.

La I serie dà i seguenti risultati:

$$
\begin{gathered}
h=30 \mathrm{~km} \\
\left(\operatorname{con} N_{1}=26\right. \text { stazioni) }
\end{gathered}
$$

\begin{tabular}{|l|l|l|}
\hline \multicolumn{1}{|c|}{$P_{n}$ (gen,) } & \multicolumn{1}{|c|}{$P_{n_{1}}$} & $P_{n_{2}}$ \\
\hline & $\mathrm{A}=50 \%$ & $\Lambda=38,5 \%$ \\
$\mathrm{~B}=57,7 \%$ & $\mathrm{~B}=11,5 \%$ & $\mathrm{~B}=34,6 \%$ \\
$\mathrm{C}=11,5 \%$ & $\mathrm{C}=15,4 \%$ \\
$\mathrm{D}=15,4 \%$ & $\mathrm{C}=15,4 \%$ & $\mathrm{D}=11,5 \%$ \\
$(\mathrm{~A}-\mathrm{B})=73,1 \%$ & $\mathrm{D}=23,1 \%$ & $(\mathrm{~A}-\mathrm{B})=73,1 \%$ \\
\hline
\end{tabular}

\begin{tabular}{|c|c|c|}
\hline$P_{n}(\operatorname{gen})$. & $P_{n_{1}}$ & $I_{n_{2}}$ \\
\hline $\begin{array}{l}\Lambda=75 \% \\
13=15 \% \\
C=10 \% \\
1)=0 \\
(A+B)=90 \%\end{array}$ & $\begin{array}{l}A=65 \% \\
B=10 \% \\
C=20 \% \\
1)=5 \% \\
(A+B)=75 \%\end{array}$ & $\begin{array}{l}A=50 \% \\
B=40 \% \\
(!=10 \% \\
I)=0 \\
(A-B)=90 \%\end{array}$ \\
\hline
\end{tabular}

La II serie dà, ovviamente, risultati migliori.

$$
\begin{gathered}
h=30 \lim \\
\left(\operatorname{con} N_{2}=20 \text { stazioni }\right)
\end{gathered}
$$


La $I I I$ serie (ottenuta tenendo conto della correzione \pm 2 s dell' $H$ ) di risultati ancora migliori (addirittura ottimi se si considerano le sole stazioni senza incertezze iniziali).

$$
h=30 \mathrm{~km}
$$

(con $X_{1}=26$ stazioni)

\begin{tabular}{|c|c|c|}
\hline$P_{n}(\operatorname{gen})$. & $P_{n_{1}}$ & $P_{n 2}$ \\
\hline $\begin{array}{l}A=80,8 \% \\
B=7,7 \% \\
C=0 \\
D=11,5 \% \\
(A+B)=88,5 \%\end{array}$ & $\begin{array}{l}A=61,5 \% \\
B=19,2 \% \\
C=7,7 \% \\
D=11,5 \% \\
(A+B)=80,7 \%\end{array}$ & $\begin{array}{l}A=46,2 \% \\
13=38,5 \% \\
C=11,5 \% \\
1)=3,9 \% \\
(A+B)=84,7 \%\end{array}$ \\
\hline
\end{tabular}

$$
h=30 \mathrm{~km}
$$

(con $N_{2}=20$ stazioni)

\begin{tabular}{|l|l|l|}
\hline \multicolumn{1}{|c|}{$P_{n}$ (gen.) } & \multicolumn{1}{|c|}{$P_{n_{1}}$} & \multicolumn{1}{c|}{$P_{n_{2}}$} \\
\hline $\mathrm{A}=100 \%$ & $\mathrm{~A}=75 \%$ & $\mathrm{~A}=50 \%$ \\
$\mathrm{~B}=0$ & $\mathrm{~B}=25 \%$ & $\mathrm{~B}=45 \%$ \\
$\mathrm{C}=0$ & $\mathrm{C}=0$ & $\mathrm{C}=5 \%$ \\
$\mathrm{I}=0$ & $\mathrm{D}=0$ & $\mathrm{D}=0$ \\
$(\mathrm{~A}+\mathrm{B})=100 \%$ & $(\mathrm{~A}+\mathrm{B})-100 \%$ & $(\mathrm{~A}+\mathrm{J})=95 \%$ \\
\hline
\end{tabular}

La $I V$ serie (dove le $P_{n}$ sono o $P_{n 1}$ o $P_{n^{\circ}}$ ) dà:

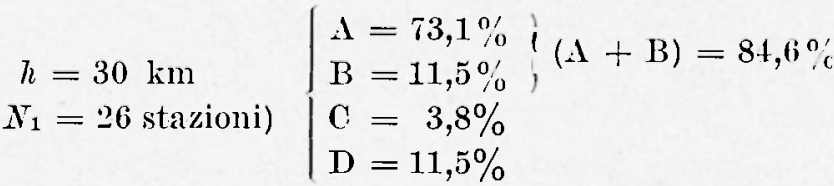

$$
\begin{aligned}
& h=30 \mathrm{~km} \\
& \left\{\begin{array}{l}
A=95 \% \\
B=5 \%
\end{array}\right\}(A+B)=100 \%
\end{aligned}
$$

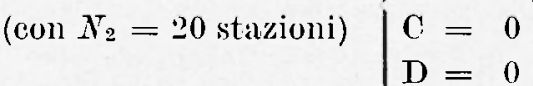

R is ultati finali. Si può dire che l'amalisi di questo terremoto confermi tutto quanto è stato detto da noi, sia a proposito dei terremoti della Sicilia, (con il rilevamento delle $P_{n 1}$ e $P_{n 2}$ ) sia anche a proposito dell'importanza che hamno gli errori $\delta \%$ e $\delta H$ alcolati 
dall'ISC. L'entità dli questi errori può essere frutto della introduzione nei calcoli, di tutte le stazioni a prescindere dai loro residni.

Le stazion i itali a ne che hamo registrato questo terremoto (Messina, Reggio C. e Roma CU) si trovano tutte classificate in A nella IV serie.

Terr. 1. 9) M. Mediterraneo. (Coste dell'Africa)

(Già esaminato, v. pag. 563).

Terr. 11. 10) Basso Timeno

$H=11^{\mathrm{n}} 09^{\mathrm{m} 2} 4^{\mathrm{s}}, 5 \pm 0 \mathrm{0}, 32$

Stazioni prese in esame fino a $20^{\circ}$.

$\mathrm{SD}=1,20$ (su 77 osservazioni)

$h_{\mathrm{ISC}}=(284 \pm 3,3) \mathrm{km}$

$h_{\text {Mosca }}=340 \mathrm{~km}$

Nagn: 4,6

$N_{1}=42$

di cui però

$2(\dot{\mathrm{S}})$

1 (S)

Stazioni senza incertezze iniziali: $N_{2}=39$

$N_{2} / N_{1}=93 \%$ (percentuale delle stazioni valide in partenza)

Terr. n. 11) Basso Tirreno

$H=02^{\mathrm{h}} 12^{\mathrm{m}} 47^{\mathrm{s}}, 9 \pm 0 \mathrm{~s}, 31$

$\mathrm{SD}=1,17$ (su 38 osservazioni)

$h_{\mathrm{TSC}}=(244 \pm 3,3) \mathrm{km}$

$h_{\text {USCGS }}=273 \mathrm{~km}$

Magn: $4,0-4,2$
Stazioni prese in esame fino a $20^{\circ}$. $N_{1}=21$ di cui però

1 (̈)

Stazioni senza incertezze iniziali. $N_{2}=20$

$N_{2} / N_{1}=95,2 \%$ (percentuale delle stazioni valide in partenza)

Osservazioni generali. Entrambi questi terremoti, come tutti quelli che caratterizzano il basso Tirreno, sono molto profoncli e non è possibile fare il confronto con le nostre dromocrone, che arrivano solo fino ad $h=60 \mathrm{~km}$.

E però molto interessante notare che i residui (O-C) calcolati dall'ISC sono tutti molto piccoli, ad eccezione di Ksara - terr. n. 10 per la quale $(\mathrm{O}-\mathrm{C})=+6^{\mathrm{s}}, 6$ denota un probabile errore di lettura, di interpretazione, di tempo, etc. La percentuale delle stazioni valide 
¿̀ elevatissima in entrambi i terremoti $\left(N_{2} / N_{1}=93 \%\right.$ - terr. 11. 10; $N_{2} / N_{1}=95,2 \%$ - terr. n. 11).

Evidentemente, data la grande profondità ipocentrale, tutte le incertezze dovute alla struttura della crosta sono eliminate. Inoltre, sempre a causa della $h$, i primi impulsi arrivano senza ambiguità e sono indicati con $i$. Molti sono stati individuati come compressioni o come dilatazioni.

Nonostante le constatazioni positive su esposte si puo notare la. differenza di valutazione delle profondità e del tempo origine calcolati da altri osservatori.

Le stazioni italia ne che hamno registrato il n. 10 sono I'Aquila, Roma MP e Trieste; e il n. 11, di Mag. più bassa è stato legistrato a Roma MIP.

Terr. 11. 12) Italia Meridionale. (Campania)

$H=06^{\mathrm{n}} 48^{\mathrm{m} 0} \mathrm{7} \mathrm{s}, 1 \pm 0^{\mathrm{s}}, 39$

$\mathrm{SD}=1,52$ (su 30 osservazioni)

$h_{\mathrm{Isc}}=(41 \pm \tau) \mathrm{km}$

$h_{\mathrm{USCGS}}=15 \mathrm{~km}$

Magn: 4,7
Stazioni prese in esame fino a $20^{\circ}$. $N_{1}=20$ di cui però 1 (Sं)

Stazioni senza incertezze iniziali: $N_{2}=19$

$N_{2} / N_{1}=95 \%$ (percentuale delle stazioni valide in partenza)

Il controllo ì stato eseguito per le dromocrone relative alle seguenti profonditì

$$
\left\{\begin{array}{l}
h_{1}=17,5 \mathrm{~km} \\
\eta_{2}=40 \mathrm{~km} \leftarrow
\end{array}\right.
$$

Osservazion i generali. I dati di partenza di questo terremoto sono abbastanza sodrlisfacenti, ed anche i residui sono contenuti entro valori accettabili (v. tab. (O-C)), meno Liublijana che ha +2 s,5. Peró le stazioni prese in esame fino a $20^{\circ}$ coprono con continuità questo intervallo solo fino a $\sim 11^{\circ}$, mentre nell'intervallo $11^{0-20}$ - vi è la sola stazione di Beni-Abbes $(1=17 \circ, 96)$.

Il controllo is stato fatto con le due dromocrone $h_{1}$ e $h_{2}$ tenuto conto del valore $15 \mathrm{~km}$ dato dall'USCGS.

I risultati sono i seguenti: 
La I serie dì

$$
\text { solo } P_{n}
$$

$$
\begin{array}{ll}
h_{1}=17,5 \mathrm{~km} & \begin{array}{l}
\mathrm{A}-15 \% \\
\mathrm{~B}=20 \% \\
\mathrm{C}=60 \% \\
\mathrm{D}=5 \%
\end{array} \\
\left.N_{1}=20 \text { stazioni }=3+\mathrm{B}\right)=35 \%
\end{array}
$$

\begin{tabular}{|c|c|c|}
\hline$P_{n}$ (gen.) & $P^{\prime} n_{1}$ & $I_{n_{2}}$ \\
\hline $\begin{array}{l}A=50 \% \\
B=35 \% \\
C=10 \% \\
D=5 \% \\
(A+B)=85 \%\end{array}$ & $\begin{array}{l}A=50 \% \\
B=20 \% \\
C=15 \% \\
D=15 \% \\
(A+B)=70 \%\end{array}$ & $\begin{array}{l}A=50 \% \\
B=25 \% \\
C=25 \% \\
D=0 \\
(A+B)=75 \%\end{array}$ \\
\hline
\end{tabular}

$h_{2}=40 \mathrm{~km}$

(con $Y_{1}=20$ stazioni)

Questa serie escluderebbe la profondità calcolata dall'USCGS.

\begin{tabular}{|c|c|c|}
\hline$P_{n}$ (gen.) & $P_{n_{1}}$ & $I_{u_{2}}$ \\
\hline $\begin{array}{l}\mathrm{A}=52,6 \% \\
\mathrm{~B}=31,6 \% \\
\mathrm{C}=10,5 \% \\
\mathrm{D}=5,3 \% \\
(\mathrm{~A}+\mathrm{B})=84,2 \%\end{array}$ & $\begin{array}{l}A=52,6 \% \\
B=21,1 \% \\
C=15,8 \% \\
1)=10,5 \% \\
(A+B)=73,7 \%\end{array}$ & $\begin{array}{l}A=52,6 \% \\
B=21,1 \% \\
C=26,3 \% \\
B=0 \\
(A-B)=73,7 \%\end{array}$ \\
\hline
\end{tabular}

La II serie conferma questi risultati, senza discostarsene troppo come è logico dato che le stazioni usate sono quasi tutte attendibili.

$$
\begin{gathered}
\text { solo } P_{n} \\
\begin{array}{c}
h_{1}=17,5 \mathrm{~km} \\
\text { (con } N_{2}=19 \text { stazioni) }
\end{array} \begin{array}{l}
\mathrm{A}=10,5 \% \\
\mathrm{~B}=21,1 \% \\
\mathrm{C}=63,2 \% \\
\mathrm{D}=5,3 \%
\end{array} \\
\text { (con } N_{2}=40 \mathrm{~km} \text { stazioni) }
\end{gathered}
$$


La III serie non è stata calcolata perché l'errore dell' $H$ ̀̀ molto piccolo.

La $I V$ serie rlà invece risultati molto sorldisfacenti (le $P_{n}$ sono o $P_{n 1}$ o $P_{n 2} ;$ questa serie è applicabile solo per $\hbar_{2}=40 \mathrm{~km}$ ).

$$
\begin{aligned}
& \left.\begin{array}{l}
h_{2}=40 \mathrm{~km} \\
\left.N_{1}=20 \text { stazioni }\right)
\end{array} \begin{array}{l}
\mathrm{A}=85 \% \\
\mathrm{~B}=15 \% \\
\mathrm{C}=0 \\
\mathrm{D}=0
\end{array}\right\}(\mathrm{A}+\mathrm{B})=100 \% \\
& h_{2}=40 \mathrm{~km} \quad\left\{\begin{array}{l}
A=89,5 \% \\
\mathrm{~B}=10,5 \%
\end{array}\right\}(A+B)=100 \% \\
& \text { (con } N_{2}=19 \text { stazioni) } \mid \begin{array}{l}
\mathrm{C}=0 \\
\mathrm{D}=0
\end{array}
\end{aligned}
$$

Ris u ltati fin a li. Dal controllo risultano convalialati i valori dell'ISC. Le stazioni vengono classificate per la maggior parte nella classe $\Lambda$. Con la IV serie si ottengono i migliori risultati.

Le sta $z$ i o n i italia ne hamno registrato quasi tutte (meno le più lontane).

Terr. n. 13) Mar Adriatico. (Coste Dalmate).

$H=23124 \mathrm{~m} 22 \mathrm{~s}, 6 \pm 0,56$

$\mathrm{SD}=1,89(\mathrm{su} 54$ osservazioni $)$

$h_{\mathrm{ISC}}=(43 \pm 8, \tilde{5}) \mathrm{km}$

$h_{\mathrm{USCGS}}=49 \mathrm{~km}$

Magn: 5
Stazioni prese in esame fino a $20^{\circ}$.

$N_{1}=38$
di cui però $\left\{\begin{array}{lc}5 & (\dot{\mathrm{S}}) \\ 2 & (\ddot{\mathrm{S}}) \\ 1 & (\dot{\mathrm{S}})\end{array}\right.$

Stazioni senza incertezze iniziali:

$$
\mathrm{N}_{2}=30
$$

$N_{\circ} / \lambda_{1}=79 \%$ (percentuale delle stazioni valicle in partenza)

Il controllo è stato eseguito per le clromocrone relative alle seguenti profondità

$$
\left\{\begin{array}{l}
h_{1}=40 \mathrm{~km} \\
h_{2}=50 \mathrm{~km}
\end{array}\right.
$$

Osservazioni generali. Il terremoto ha rati di partenza abbastanza attendibili, gli errori di $H$, di $h$, e l'SD sono piccoli, però solo il $79 \%$ delle stazioni sono valide in partenza. 
Le 1 fino a $20^{\circ}$ sono coperte con una discreta continuita.

te due $h$ controllatie vogliono tener conto dei valori calcolati dall'ISC o dall'USCGS.

I risultati sono i seguenti:

La I serie dà:

$h_{1}=40 \mathrm{~km}$

(ron $X_{1}=38$ stazioni)

\begin{tabular}{|l|l|l|}
\hline$P_{n \text { (gen.) }}$ & \multicolumn{1}{|c|}{$P_{n_{1}}$} & \multicolumn{1}{c|}{$P_{n, 2}$} \\
\hline & $\mathrm{A}=52,6 \%$ & $\mathrm{~A}=26,3 \%$ \\
$\mathrm{~A}=47,4 \%$ & $\mathrm{~B}=18,4 \%$ & $\mathrm{~B}=34,2 \%$ \\
$\mathrm{~B}=28,9 \%$ & $\mathrm{C}=15,8 \%$ & $\mathrm{C}=28,9 \%$ \\
$\mathrm{D}=15,8 \% \%$ & $\mathrm{D}=1 \mathrm{O}=10,5 \%$ \\
$(\mathrm{~A}-\mathrm{A}-\mathrm{B})=76,3 \%$ & $(\mathrm{~A}+\mathrm{B})=7 \mathrm{~B})$ & $(\mathrm{A}+\mathrm{B})=60,5 \%$ \\
\hline
\end{tabular}

$h_{2}=50 \mathrm{~km}$

(con $N_{1}=38$ stazioni)

\begin{tabular}{|c|c|c|}
\hline$P_{n}($ gron. $)$ & $P_{u_{1}}$ & $I_{n_{2}}$ \\
\hline $\begin{array}{l}A=63,2 \% \\
B=10,5 \% \\
(C=21,1 \% \\
1)=5,3 \% \\
(A+B)=73,7 \%\end{array}$ & $\begin{array}{l}A=55,3 \% \\
B=13,2 \% \\
(1=21,1 \% \\
1)=10,5 \% \\
(A+B)=68,5 \%\end{array}$ & $\begin{array}{l}A=13,2 \% \\
B=0 \\
C=44,7 \% \\
1)=42,1 \% \\
(A+B)=13,2 \%\end{array}$ \\
\hline
\end{tabular}

Lit II serie dì:

$$
h_{1}=40 \mathrm{~km}
$$

(con $N_{2}=30$ stazioni)

\begin{tabular}{|c|c|c|}
\hline$I_{n}$ (gen.) & $P_{n_{1}}$ & $P_{n 2}$ \\
\hline $\begin{array}{l}A=60 \% \\
13=33,3 \% \\
C=6,7 \% \\
1)=0 \\
(A+13)=93,3 \%\end{array}$ & $\begin{array}{l}A=66,7 \% \\
B=13,3 \% \\
(\vdots=16,7 \% \\
D=3,3 \% \\
(A-13)=80 \%\end{array}$ & $\begin{array}{l}A=26,7 \% \\
B=40 \% \\
C=33,3 \% \\
B=0 \\
(A=B)=66,7 \%\end{array}$ \\
\hline
\end{tabular}


$h_{2}=50 \mathrm{kin}$

(con $\hat{S}_{2}=30$ stazioni)

\begin{tabular}{|l|ll|}
\hline \multicolumn{1}{|c|}{$P_{n}$ (gen.) } & \multicolumn{1}{|c|}{$I_{n_{1}}$} & $I_{n_{2}}$ \\
\hline & & \\
$\mathrm{A}=73,3 \%$ & $\mathrm{~A}=70 \%$ & $\mathrm{~A}=10 \%$ \\
$\mathrm{~B}=13,3 \%$ & $\mathrm{~B}=10 \%$ & $\mathrm{~B}=0$ \\
$\mathrm{C}=13,3 \%$ & $\mathrm{C}=20 \%$ & $\mathrm{C}=46,7 \%$ \\
$\mathrm{~J})=0$ & $\mathrm{D}=0$ & $\mathrm{O}=43,3 \%$ \\
$(\mathrm{~A}+\mathrm{B})=86,6 \%$ & $(\mathrm{~A}+\mathrm{B})=80 \%$ & $(\mathrm{~A}+\mathrm{B})=10 \%$ \\
\hline
\end{tabular}

Questa II serie costituisce, come al solito, un ovvio notevole miglioramento rispetto alla I serie e darebbe una preferenza ai risultati per $h=40 \mathrm{~km}$.

La III serie non è stata calcolata dato il piccolo errore di $H( \pm 0$ s, 56$)$.

La $I V$ serie (le $P_{n}$ sono o $P_{n 1}$ o $P_{n 2}$ ) dà i seguenti risultati:

$h_{1}=40 \mathrm{~km}$.

$$
\operatorname{con} N_{1}=38 \text { stazioni }\left\{\begin{array}{l}
A=73,7 \% \\
\mathrm{~B}=13,2 \% \\
\mathrm{C}=7,9 \% \\
\mathrm{D}=5,3 \%
\end{array}\right\}(\mathrm{A}+\mathrm{B})=86,9 \%
$$

$$
\operatorname{con} N_{2}=30 \text { stazioni }\left\{\begin{array}{l}
\mathrm{A}=86,7 \% \\
\mathrm{~B}=10 \% \\
\mathrm{C}=3,3 \% \\
\mathrm{D}=0
\end{array}\right\}(\mathrm{A}+\mathrm{B})=96,7 \%
$$

$h_{2}=50 \mathrm{~km}$

$$
\operatorname{con} N_{1}=38 \text { stazioni }\left\{\begin{array}{l}
\mathrm{A}=68,4 \% \\
\mathrm{~B}=13,2 \% \\
\mathrm{C}=15,8 \% \\
\mathrm{D}=2,6 \%
\end{array}\right\}(\mathrm{A}+\mathrm{B})=81,6 \%
$$

$$
\text { con } N_{2}=30 \text { stazioni }\left\{\begin{array}{l}
A=80 \% \\
\mathrm{~B}=10 \% \\
\mathrm{C}=10 \% \\
\mathrm{D}=0
\end{array}\right\}(\mathrm{A}+\mathrm{B})=90 \%
$$

Ris u ltati finali. I risultati ottenuti con la IV serie, pur essendo molto buoni per entrambe le $h$ controllate, confermano la preferenza per $h=40 \mathrm{~km}$ delineatasi nella II serie. 
Le stazion i italiane che hamno registrato questo terremoto sono Roma MIP e Trieste, ma Roma MP (che ha un residuo $(\mathrm{O}-\mathrm{C})=+6 \mathrm{~s})$ ha iniziato certamente la registrazione con un'onda successiva alla $P_{n}$.

Terr. n. 14) Mare Ionio. (Coste Calabre)

$H=10^{\mathrm{h}} 45^{\mathrm{m} 2 \mathrm{2}} \mathrm{s}, 1 \pm 0^{\mathrm{s}}, 70$

$\mathrm{SD}=1,80($ su 54 osservazioni $)$

$h_{\mathrm{tsc}}=(53 \pm 8) \mathrm{km}$

$h_{\mathrm{BCIS}}=80 \mathrm{~km}$

Mag: 4,3
Stazioni prese in esame fino a $20^{\circ}$.

\begin{tabular}{l|lc}
$N_{\mathrm{t}}=33$ & $(\dot{\mathrm{S}})$ \\
0 & $(\ddot{\mathrm{S}})$ \\
3 & $(\dot{\mathrm{S}})$
\end{tabular}

Stazioni senza incertezze iniziali: $N_{2}=22$

$N_{2} / N_{1}=66,7 \%$ (percentuale relle stazioni valicle in partenza)

Il controllo è stato eseguito per la dromocrona relativa alla seguente profonditì

$$
h=50 \mathrm{~km} \leftarrow
$$

Osservazion generali. In questo terremoto c'è da notare che anche se $\mathrm{i}$ dati di partenza dell'ISC sono abbastanza buoni (sia le correzioni di $H$, di $h$, sia l'SD) tuttavia la presenza di molte stazioni con incertezze iniziali (solo il $66,7 \%$ delle stazioni esaminate sono valide in partenza) disturba alquanto le osservazioni che se ne possono trarre. Inoltre i valori che Mosea e il BCTS danno per $H$, differiscono notevolmente da quello dell'TSC (v. parte alta tab. n. 1) come pure c'è notevole discordanza tra l' $h_{1 \mathrm{sc}}$ e l' $h_{\mathrm{BC} \mathrm{s}}$.

Tutte queste considerazioni famno prevedere ahe il controllo effettuato sui dati dell'ISC non sarà molto soddisfacente. Tuttavia per uniformità di procerlimento ci atterremo ad essi e il controllo verrà fatto per l'unica profondità $h=50 \mathrm{~km}$. Ise stazioni coprono con continuità le $A$, arrivando però solo fino a Beni- $\Lambda$ bbes $(\Delta=170,2 \pi)$ prima dei $20^{\circ}$. 
Là I serie dà i seguenti risultati:

$$
\begin{gathered}
h=50 \mathrm{~km} \\
\text { (con } \gamma_{1}=33 \text { stazioni) }
\end{gathered}
$$

\begin{tabular}{|c|c|c|}
\hline$P_{n}^{\prime}(\operatorname{sen})$. & $I_{n_{1}}^{\prime}$ & $P_{n ! 2}$ \\
\hline $\begin{array}{l}A=60,6 \% \\
B=9,1 \% \\
C=21,2 \% \\
1)=9,1 \% \\
(A-B)=69,7 \%\end{array}$ & $\begin{array}{l}A=45,5 \% \\
B=30,3 \% \\
C=12,1 \% \\
1)=12,1 \% \\
(A+B)=75,8 \%\end{array}$ & $\begin{array}{l}A=12,1 \% \\
B=15,2 \% \\
C=27,3 \% \\
1)=45,5 \% \\
(A-B)=27,3 \%\end{array}$ \\
\hline
\end{tabular}

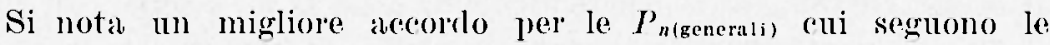
$P_{n 1}$; le $P_{n 2}$ invece sono quasi inesistenti. Se invece dei soli $A$ si consirlerano gli $(\Lambda+B)$, per le $P_{n 1}$ si hanno i migliori risultati.

La II serie, naturalmente, dì risultati miglior'i

\begin{tabular}{|c|c|c|}
\hline$P_{n}(\boldsymbol{u}(\mathrm{n}))$. & $I_{n_{1}}$ & $I_{n_{2}}^{\prime}$ \\
\hline $\begin{array}{l}A=90,9 \% \\
B=4,5 \% \\
C=4,5 \% \\
1)=0 \\
(A+B)=95,4 \%\end{array}$ & $\begin{array}{l}A==63,6 \% \\
B=27,3 \% \\
C=9,1 \% \\
D=0 \\
(A+B)=90,9 \%\end{array}$ & $\begin{array}{l}A=0 \\
B=18,2 \% \\
C=40,9 \% \\
I)=40,9 \% \\
(A+B)=18,2 \%\end{array}$ \\
\hline
\end{tabular}

$$
\begin{gathered}
h=50 \mathrm{~km} \\
\left(\operatorname{con} N_{2}=22\right. \text { stazioni) }
\end{gathered}
$$

però permane la situazione di netta preferenza per le $P_{n \text { (gencrall). }}$

Riteniamo che questo trovi la sua giustificazione nelle incertezze rivelate clagli $H$ e clall'h trovate dagli altri centri di osservazione.

La $I I I$ serie non ì stata calcolata: il piccolo erore clell $H( \pm 0$ s,70) (relativamente all'TSC) comporta solo oscillazioni di $A$ al di sotto di $10 \mathrm{~km}$ (v. grafico fig. 3) per cui la situazione resta pressoche immutata. 
La $I V$ serie (le $P_{n}$ sono o $P_{n 1}$ o $P_{n 2}$ ) dà:

$$
\begin{aligned}
& \begin{array}{l}
h=50 \mathrm{~km} \\
\left.N_{1}=33 \text { stazioni }\right)
\end{array}\left\{\begin{array}{l}
\mathrm{A}=57,6 \% \\
\mathrm{~B}=39,4 \% \\
\mathrm{C}=3,0 \% \\
\mathrm{C}=0
\end{array}\right\}(\mathrm{A}+\mathrm{B})=97 \% \\
& \left.h=50 \mathrm{~km} \quad \begin{array}{l}
\mathrm{A}=63,6 \% \\
\mathrm{~B}=36,4 \%
\end{array}\right\}(A+\mathrm{B})=100 \%
\end{aligned}
$$

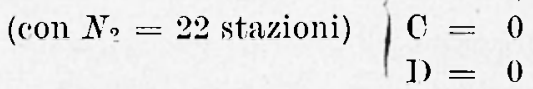

In quest'ultima serie, quella calcolata con $N_{2}$ stazioni, scompaiono tutti i 1$)$ e tutti i $($. L' $(A+B)$ raggiunge il massimo valore $(100 \%)$, però il contributo dei $B$ è notevole.

$\mathrm{R}$ is ultati finali . La profondità $h=50 \mathrm{~km}$ trova abbastanza credito, specialmente all'esame della II e IV serie. (Gli $80 \mathrm{~km}$ dell' $h_{\mathrm{BC}}$ sono fuori della nostra possibilita di controllo).

Delle stazioni italiane Roma MP e Trieste hanno registrato questo sisma (di $M=4,3)$; però a Trieste le $P_{n}$ arrivano in anticipo $\left((\mathrm{O}-\mathrm{C})=-2^{\mathrm{s}}, 4\right)$.

Terr. n. 15) Jugoslavia

$$
\begin{aligned}
& H=08^{\mathrm{n}} 10^{\mathrm{m}} 58^{\mathrm{s}}, 2 \text { 土 } 0^{\mathrm{s}}, 15 \\
& \mathrm{SI})=2,07 \text { (su } 244 \text { osservazioni) } \\
& h_{\mathrm{Isc}}=33 \mathrm{~km} \\
& \text { Magn: } 5,3-6,7 \\
& \begin{array}{l|l}
N_{1}=70 & 22(\dot{\mathrm{S}})
\end{array} \\
& \text { di cui però | } 8(\ddot{\mathrm{S}}) \\
& N_{2}=40
\end{aligned}
$$

Il controllo è stato eseguito per la clromocrona relativa alla seguente profondita

$$
h=30 \mathrm{~km} \leftarrow-
$$

Os servazioni generali. Il terremoto, data la sua elevata $M$, i stato largamente registrato; tuttavia solo il $57 \%$ delle stazioni esaminate ha dati validi in partenza. 
Le stazioni coprono con continuitì tutto lintervallo delle 1 fino a $20 \%$.

La 7 data dai vari centri di elaborazione concorda con $h=33 \mathrm{~km}$ calcolato dall'ISC; poiché questo valore ì dato senza correzione, il controllo è stato fatto per l'unica profonclita $h=30 \mathrm{~km}$.

I risultati sono i seguenti:

La I serie dà:

$$
\begin{gathered}
h=30 \mathrm{~km} \\
\left(\text { con } N_{1}-70\right. \text { stazioni) }
\end{gathered}
$$

\begin{tabular}{|c|c|c|}
\hline$P_{n}($ gren. $)$ & $I_{n_{1}}^{\prime}$ & $P_{n_{2}}$ \\
\hline $\begin{array}{l}A=34,3 \% \\
B=28,6 \% \\
C=28,6 \% \\
1)=8,6 \% \\
(A+B)=62,9 \%\end{array}$ & $\begin{array}{l}A=52,9 \% \\
B=24,3 \% \\
C=20 \% \\
D=2,9 \% \\
(A+B)=77,2 \%\end{array}$ & $\begin{array}{l}A=25,7 \% \\
B=8,6 \% \\
C=35,7 \% \\
D=30 \% \\
(A-B)=34,3 \%\end{array}$ \\
\hline
\end{tabular}

E molto evidente il forte contributo dato ai $\mathrm{C}$ e ai $\mathrm{D}$ dalle numerose stazioni incerte.

Ia $I I$ serie dì:

$$
\begin{gathered}
h=30 \mathrm{~km} \\
\left(\operatorname{con} N_{2}=40 \text { stazioni }\right)
\end{gathered}
$$

\begin{tabular}{|l|l|l|}
\hline \multicolumn{1}{|c|}{$P_{n}$ (gen.) } & \multicolumn{1}{|c|}{$P_{n_{1}}$} & \multicolumn{1}{c|}{$P_{n_{2}}$} \\
\hline $\mathrm{A}=60 \%$ & $\mathrm{~A}=75 \%$ & $\mathrm{~A}=42,5 \%$ \\
$\mathrm{~B}=35 \%$ & $\mathrm{~B}=7,5 \%$ & $\mathrm{~B}=15 \%$ \\
$\mathrm{C}=5 \%$ & $\mathrm{C}=17,5 \%$ & $\mathrm{C}=42.5 \%$ \\
$\mathrm{D}=0$ & $\mathrm{D}=0$ & $\mathrm{I}=0$ \\
$(\mathrm{~A}+\mathrm{B})=95 \%$ & $(\mathrm{~A}+\mathrm{B})=82,5 \%$ & $(\mathrm{~A}-\mathrm{B})=5 \mathrm{5}, 5 \%$ \\
\hline
\end{tabular}

Questo controllo porta ad un miglioramento generale in cui prevalgono le $A$ delle $P_{n 1}$ (con il $75_{\%}^{\circ}$ ), però il migliore $(A+B)$ si ha con le $P_{n}$ (generali) $(\mathrm{A}+\mathrm{B})=95 \%$ ).

La $I I I$ serie non è stata calcolata perché la correzione di $H$ is solo $\pm 0 s, 15$. 
La $I V$ serie (le $P_{n}$ sono o $P_{n 1}$ o $P_{n 2}$ ) dà risultati molto buoni con le 70 stazioni, e arldirittura ottimi con le sole 40 .

$$
\begin{aligned}
& \begin{array}{c|l}
\hbar=30 \mathrm{~km} & \begin{array}{l}
\lambda=68,6 \% \\
\mathrm{~B}=18,6 \%
\end{array} \\
\left.N_{1}=70 \text { stazioni }\right) & \mathrm{C}=11,4 \% \\
\mathrm{D}=1,4 \%
\end{array} \\
& \left.h=30 \mathrm{~km} \quad \begin{array}{l}
\Lambda=100 \% \\
\mathrm{~B}=0
\end{array}\right\}(A+\mathrm{B})=100 \% \\
& \text { (con } N_{2}=40 \text { stazioni) } \mid \begin{array}{ll}
\mathrm{C}=0 \\
\mathrm{D})=0
\end{array}
\end{aligned}
$$

Tutte le 40 stazioni sono di classe $\Lambda$.

Risultati finali. Valislo dunque il controllo, specialmente quello relativo alla IV serie.

Le sta z i on i it a l a ne hamno registrato quasi tutte classificandosi tra le $A$, alcune come $P_{n 1}$ altre come $P_{n 2}$. Fanno eccezione Messina ING e Pavia che lanno entrambe un anticipo nei tempi di arrivo.

Terr. n. 16) Jugoslavia

$H=02{ }^{h} \tilde{\jmath} 9^{\mathrm{m}} 57 \mathrm{~s}, 3 \pm 0 \mathrm{~s}, 32$

$\mathrm{SI})=1,10$ (su 30 osservazioni)

$h_{1 \mathrm{SC}}=h_{\mathrm{LscGs}}=16 \mathrm{~km}$

Mạn: 4,2
Stazioni prese in esame fino a $20^{\circ}$. $N_{1}-16$ rli cui però $\left.1 . \ddot{\mathrm{S}}\right)$

Stazioni senza incertezze iniziali:

$$
N_{2}=15
$$

$N_{2} / N_{1}=94 \%$ (percentuale rlelle stazioni valide in partenza)

Il controllo è stato eseguito per la rlromocrona relativa alla seguente profonclità

$$
h=17,5 \mathrm{~km} \leftarrow
$$

Osservazioni generali. I rati di partenza rli questo terremoto sono abbastanza uniformi. C'è da notare tuttavia che mentre le brevi distanze sono coperte con continuità fino ai $\angle=6^{\circ}, 80$ ali Lanslevillard rlopo questa stazione e'e un vuoto fino ai $\Delta=74^{\circ}, 60$ (v. boll. ISC) quindi i dati di partenza, anche se apparentemente uniformi, devono per forza risentire di questa assenza di dati intermedi. 
La I serie da i seguenti risultati (il controllo è fatto solo sulle

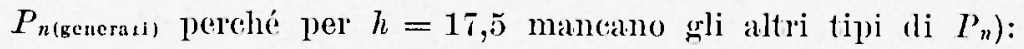

$$
\begin{gathered}
P_{n} \\
h=17,5 \mathrm{~km}
\end{gathered} \quad \begin{aligned}
& \mathrm{A}-18,8 \% \\
& \mathrm{~B}=50 \% \\
& \mathrm{C}=25 \% \\
& \mathrm{I}=6,3 \%
\end{aligned}
$$

La II serie ovviamente dà risultati migliori perché scompaniono i 1$)$

$$
\begin{aligned}
& \left.h=17,5 \mathrm{~km} \quad \begin{array}{l}
A=20 \% \\
B=53,3 \%
\end{array}\right\}(A+B)=73,3 \%
\end{aligned}
$$

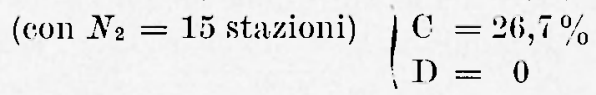

tuttavia in entrambe le serie predomina la classe B mentre scarseggia la classe $\mathrm{A}$.

La $I I I$ serie non è stata calcolata perché la correzione di $H$ è piccola ( \pm 0 s, 32$)$.

La $I V$ serie non è stata calcolata perché per $h=17,5 \mathrm{~km}$ ei sono solo le $P_{n}$.

$R$ is ultat i f in a $1 \mathrm{i}$. Il controllo fatto per questo terremoto là risultati poco soddisfacenti, ma daaltra parte le premesse lo facevano prevedere: le stazioni disponibili sono troppo poche e distribuite entro distanze che possono essere riferite solo alla prima parte della dromocrona.

Delle stazion i t a lia ne ha registrato Trieste, classificandosi in $B$.

Terr. 11. 17) Mare Ionio (presso Grecia)

$H=00^{\mathrm{h}} 58^{\mathrm{m}} 45^{\mathrm{s}} \pm 2 \mathrm{~s}, 0$

$\mathrm{SI})=3,58(\mathrm{su} 6.2$ osservazioni $)$

$h_{\mathrm{ISc}}=(11 \pm 13) \mathrm{km}$

$h_{\mathrm{uscas}}=14 \mathrm{~km}$

Nag: 4,6
Stazioni prese in esame fino a $20^{\circ}$.

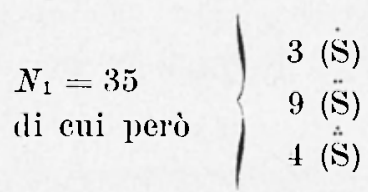

Stazioni senza incertezze iniziali: $N_{2}=19$

$N_{2} / N_{1}=54,3 \%$ (percentuale delle stazioni valide in partenzal) 
Il controllo ì stato eseguito per le dromocrone relative alle seguenti profondità

$$
\left\{\begin{array}{l}
h_{1}=5 \mathrm{~km} \\
h_{2}=17,5 \mathrm{~km}
\end{array}\right.
$$

Osservazioni generali. Questo terremoto ì stato registrato da un gran numero di stazioni: quelle comprese entro i primi $20{ }^{\circ}$ coprono con continuità l'intervallo delle $A$, però solo il 54\% di esse offrono garanzie di sicurezza fin dall'inizio. Ise restanti hammo resilui (O-C) anche molto elevati (v. tabella n. 2). Queste notevoli incertezze sono denunciate anche dallerrore standard SD (3s,58), dalla correzione dell' $H( \pm 2 \mathrm{~s}, 0)$ e dalla correzione dell\% ( $\pm 13 \mathrm{~km}$ ).

Per le dromocrone a profondità $h_{1}=5 \mathrm{~km}$ e $h_{2}=17,5 \mathrm{~km}$ esistono solo le $P_{n}{ }_{n}$ generali.

La I serie da i seguenti risultati:

$$
\begin{gathered}
h_{1}=5 \mathrm{~km} \\
\text { (con } N_{1}=35 \text { stazioni) } \\
\begin{array}{c}
\mathrm{A}=14,3 \% \\
\mathrm{~B}=8,6 \% \\
\mathrm{C}=42,9 \% \\
\mathrm{D}=34,3 \%
\end{array} \\
h_{2}=17,5 \mathrm{~km} \\
\left.N_{1}=35 \text { stazioni }\right)
\end{gathered} \begin{aligned}
& \mathrm{A}=51,4 \% \\
& \mathrm{~B}=8,6 \% \\
& \mathrm{C}=8,6 \% \\
& \mathrm{D}=31,4 \%
\end{aligned}
$$

Da questo primo esame emerge nua preferenza netta per $h_{2}=17,5 \mathrm{~km}$ (le classi ( e I), sono ovviamente, molto consistenti).

La II serie da:

$$
\begin{gathered}
h_{1}=5 \mathrm{~km} \\
\text { (con } N_{2}=19 \text { stazioni) } \\
\begin{array}{c}
\mathrm{P}=10,5 \% \\
\mathrm{~B}=10,5 \% \\
\mathrm{C}=63,2 \% \\
\mathrm{D}=15,8 \%
\end{array} \\
P_{\prime \prime} \\
\begin{array}{c}
\mathrm{A}=89,5 \% \\
h_{2}=17,5 \mathrm{~km}
\end{array} \\
\begin{array}{c}
\mathrm{B}=10,5 \% \\
\mathrm{C}=0
\end{array} \\
\mathrm{I}=0
\end{gathered}
$$


Per $h=17,5 \mathrm{~km}$ si hanno risultati preferibili in maniera sempre più evidente: scompaiono infatti tutte le classi (C e D e la somma $(A+B)$ raggiunge il $100 \%$.

La III serie (calcolata tenendo conto dellincidenza dei \pm 2 s,0 dell' $H$ sulle $\Delta$ in base ai grafici di fig. 2 e di fig. 3) dà:

$$
\begin{aligned}
& \left.\begin{array}{l|l}
h_{1}=5 \mathrm{~km} & \begin{array}{l}
\mathrm{A}=45,7 \% \\
\mathrm{~B}=22,9 \%
\end{array}
\end{array}\right\}(\mathrm{A}+\mathrm{B})=68,6 \% \\
& \begin{array}{l|l}
\text { (con } N_{1}=35 \text { stazioni) } & \begin{array}{l}
\mathrm{C}=14,3 \% \\
\mathrm{D}=17,1 \%
\end{array}
\end{array} \\
& \left.h_{1}=5 \mathrm{~km} \quad \begin{array}{l}
\Lambda=52,6 \% \\
\mathrm{~B}=36,8 \%
\end{array}\right\}(\Lambda+B)=89,4 \% \\
& \begin{array}{l|l}
\text { (con } N_{2}=19 \text { stazioni) } & \begin{array}{l}
\mathrm{C}=5,3 \% \\
\mathrm{D}=5,3 \%
\end{array}
\end{array} \\
& \left.h_{2}=17,5 \mathrm{~km} \quad \begin{array}{l}
\mathrm{A}=62,9 \% \\
\mathrm{~B}=8,6 \%
\end{array}\right\}(\mathrm{A}+\mathrm{B})=71,5 \% \\
& \text { (con } N_{1}=35 \text { stazioni) } \mid \begin{array}{l}
\mathrm{C}=17,1 \% \\
\mathrm{D}=11,4 \%
\end{array} \\
& \left.\begin{array}{c|l}
\hbar_{2}=17,5 \mathrm{~km} & \begin{array}{l}
\mathrm{A}=100 \% \\
\mathrm{~B}=0 \\
\mathrm{C}=0 \\
\mathrm{D}=0
\end{array}
\end{array}\right\}(\Lambda+\mathrm{B})=100 \%
\end{aligned}
$$

Con questa serie le cose migliorano notevolmente anche per $h_{1}=5 \mathrm{~km}$; per questa sembra evidenziarsi una certa probabilita (specie per $\mathrm{N}_{2}$ stazioni); tuttavia nettamente migliori sono i risultati per $h_{2}=17,5 \mathrm{~km}$ dove le 19 stazioni senza incertezze iniziali vengono tutte assegnate alla classe $\mathrm{A}$.

Eे evidente il peso che hanno nei nostri controlli le stazioni con residui elevati, e ciò convalida ancora una volta l'osservazione che abbiamo fatto a proposito dell'incidenza degli errori di lettura o di interpretazione.

La IV serie non è stata calcolata perché nelle due dromocrone $\left(h_{1}=5 \mathrm{~km}, h_{2}=17,5 \mathrm{~km}\right)$ non e'è distinzione tra le $P_{n 1}$ e le $P_{n 2}$.

I risultati migliori si hamno dunque per

$$
h_{2}=17,5 \mathrm{~km}
$$


Iue s t a z i o n i it a li a n e ehe hamno registrato il sisma sono Messina ING, Roma MP e Trieste; ma Trieste che ha $(0-\mathrm{C})=-6 \mathrm{~s}, 2$ (v. tab. resiclui) è incorsa eviclentemente in qualche errore.

Ter'r. 11. 18) (rrecia

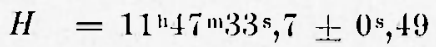

$\mathrm{SI}=2,01$ (su 45 osservazioni)

$h_{\mathrm{ISC}}=:(67 \pm 6,6) \mathrm{km}$

$h_{\mathrm{uscas}}=6+\mathrm{km}$

Magn: $4,3-4, \tilde{5}$

Stazioni prese in exame fino a $20^{\circ}$.

\begin{tabular}{l|ll}
$N_{1}=20$ & $2(\mathbf{S})$ \\
di cui però & $(\ddot{\mathbf{S}})$ \\
1 & $(\dot{\mathrm{S}})$
\end{tabular}

Stazioni senza incertezze iniziali: $N_{2}=16$

$N_{2} / N_{1}=80 \%$ (percentuale delle stazioni valicle in partenza)

Il controllo o stato eseguito per la dromocrona relativa alla seguente profondita

$$
h=60 \mathrm{lim} \leftarrow
$$

Osservazioni generali. Le stazioni considerate coprono l'intervallo delle $A$ con soddisfacente continuità, fino ai $15^{\circ}, 4$ di Eilat dopo la quale non compaiono altre stazioni fino oltre i $20^{\circ}$. Tato il buon accordo tra 1 ' $h_{\text {ISc }}$ e l' $h_{\text {Lsces }}$ e il piccolo errore relativo, abbiamo controllato solo con la dromocrona $h=60 \mathrm{~km}$.

Ia I serie dà i seguenti risultati:

$$
\begin{gathered}
h=60 \mathrm{~km} \\
\left(\text { con } N_{1}=20 \text { stazioni }\right)
\end{gathered}
$$

\begin{tabular}{|c|c|c|}
\hline$P_{n}($ gren.) & $P_{n_{1}}$ & $I_{n 2}$ \\
\hline $\begin{array}{l}A=150 \% \\
B=10 \% \\
C=5 \% \\
D=20 \% \\
(A+B)=75 \%\end{array}$ & $\begin{array}{l}A=50 \% \\
B=15 \% \\
(C=20 \% \\
B=15 \% \\
(A+B)=65 \%\end{array}$ & $\begin{array}{l}A=45 \% \\
B=25 \% \\
C=20 \% \\
D=10 \% \\
(A+B)=70 \%\end{array}$ \\
\hline
\end{tabular}


La II serie dà ovviamente risultati più sorldisfacenti:

$$
h=60 \mathrm{k} \| \mathrm{l}
$$

(con $S_{2}=16$ stazioni)

\begin{tabular}{|c|c|c|}
\hline$P_{n}$ (gen.) & $I_{n_{1}}^{\prime}$ & $I_{n_{2}}$ \\
\hline $\begin{array}{l}A=81,3 \% \\
B=12,5 \% \\
\vdots=6,3 \% \\
1 D=0 \\
A+B)=93,8 \%\end{array}$ & $\begin{array}{l}A=62,5 \% \\
B=18,8 \% \\
C=18,8 \% \\
J=0 \\
(A+B)=81,3 \%\end{array}$ & $\begin{array}{l}A=56,3 \% \\
B=31,3 \% \\
C=12,5 \% \\
1=0 \\
(A+B)=87,6 \%\end{array}$ \\
\hline
\end{tabular}

scompaiono tutti $\mathrm{i} D$ e le $\mathrm{A}$ aumentano notevolmente, specialmente quelle relative alle $P_{n}$ generali.

La III serie non è stata calcolata perché l'errore di $H$ ì solo $\pm 0 \mathrm{~s}, 49$.

La $I V$ serie (le $P_{n}$ sono $P_{n 1} \circ P_{n 2}$ ) dà risultati ottimi, specie considerando le sole stazioni valide in partenza che vengono assegnate tutte alla classe $A$ :

$$
\begin{aligned}
& \left.h=60 \mathrm{~km} \quad \begin{array}{l}
A=80 \% \\
\mathrm{~B}=0 \%
\end{array}\right\}(A+B)=80 \% \\
& \text { (con } N_{1}=20 \text { stazioni) } \quad \mathrm{C}=15 \% \\
& \mathrm{I}=5 \% \\
& \left.h=60 \mathrm{~km} \quad \begin{array}{l}
A=100 \% \\
B=0
\end{array}\right\}(A+B)=100 \% \\
& \text { (con } N_{2}=16 \text { stazioni) } \mid \begin{array}{l}
\mathrm{C}=0 \\
\mathrm{D}=0
\end{array}
\end{aligned}
$$

R is ultati finali. Ia validita della nostra dromocrona per $h=60 \mathrm{~km}$ trova qui piena conferma, specialmente con il controllo rell'ultima serie.

Le stazioni italiane che hamno registrato sono le seguenti: LiAquila, Roma MIP (entrambe assegnate alla (classe $A$ ) e Messina U.; questa inclica come $P_{n}$ un'onda probabilmente successiva poichè $(\mathrm{O}-\mathrm{C})=+3^{\mathrm{s}}, \mathrm{t}$. 
Terr. 11. 19) Albania

$H=12^{n} 44^{\mathrm{m}} 42^{\mathrm{s}}, 7 \pm 0 \mathrm{~s}, 70$

$\mathrm{SD}=1,51$ (su 168 osselvazioni)

$h_{\mathrm{rsc}}=(16 \pm \tilde{5}, 4) \mathrm{km}$

Magn: 5,3

Stazioni prese in esame fino a $20^{\circ}$.

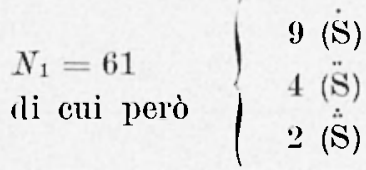

Stazioni senza incertezze iniziali: $N_{2}=46$

$N_{2} / N_{1}=75,4 \%$ (percentuale relle stazioni valirle in partenza)

Il controllo è stato eseguito per la dromocrona relativa alla seguente profonclità

$$
h=17,5 \mathrm{~km} \leftarrow
$$

Osservazioni generali. Natualmente i controlli li faremo, come stabilito, riferendoci all' $H_{\text {ISc }}$, però è da notare che altri centri di osservazione damno per $H$ valori differenti: l' $H$ di Atene differisce di $\sim 5$ secondi (v. parte alta tab. 1). Sono da preverlere risultati poco sorldisfacenti anche per l'alta percentuale delle stazioni incerte (v. tab. n. :2).

Le stazioni coprono con continuita tutte le $A$ fino a 200 . I'h $=17,5 \mathrm{~km}$ la le sole $P_{n}$ generali.

I controlli portano ai seguenti risultati:

La I serie dà:

$$
\left.\begin{array}{c}
P_{n} \\
h=17,5 \mathrm{~km} \\
\text { (con } N_{1}=61 \text { stazioni) } \\
\mathrm{A}=45,9 \% \\
\mathrm{~B}=23,0 \%
\end{array}\right\}\left(\begin{array}{l}
\mathrm{C}=21,3 \% \\
\mathrm{I})=9,8 \%
\end{array}\right.
$$

Meglio naturalmente, la II serie:

$$
\text { (con } N_{2}=46 \text { stazioni) } \quad \begin{aligned}
& \mathrm{A}=58,7 \% \\
& \mathrm{~B}=21,7 \% \\
& \mathrm{C}=19,6 \% \\
& \mathrm{I})=0
\end{aligned}
$$


Aleune stazioni assegnate alla classe $D$ sono qui passate alle classi superiori, tuttavia resta ancora il $\sim 20 \%$ di stazioni assegnate alla classe C. Il 60\% delle 46 stazioni è assegmata alla classe $\Lambda$.

Ia III scrie non è stata calcolata, dato il piccolo errore attribuito and $H( \pm 0$ s, 70$)$.

La IV serie non è stata calcolata perché per $h=17,5 \mathrm{~km}$ ai sono solo le $P_{n}$ generali.

Ris l l t a t i f in a li. Buono, non ottimo, il controllo fatto per $h=17,5 \mathrm{~km}$ (II serie), né d'altra parte c'ela da attendersi di meglio, viste le osservazioni preliminari.

Le stazion i italiane hammo quasi tutte registrato il sisma: Messina ING e Reggio C. (che però registrano onde successive alle $\left.P_{n}\right)$; Parlova e Trieste che sono assegnate alla classe $A$; Roma MP e Taranto che sono assegnate alla classe C; l'Aquila che, con uno scarto $(\mathrm{O}-\mathrm{C})=+5^{\mathrm{s}}, 7$, ha segnalato come $P_{n}$ onde che evidentemente sono successive.

Terr. n. 20) Mediterraneo orientale (al largo di Creta)

$H=13^{\mathrm{n}} 33^{\mathrm{m}} \tilde{5} 3^{\mathrm{s}} \pm 0 \mathrm{~s}, 96$

$\mathrm{SD})=2,61$ (su 34 osservazioni)

$\hbar_{1 \mathrm{sc}}=18 \mathrm{~km}$

Stazioni prese in esame lino a $20^{\circ}$.

\begin{tabular}{l|l}
$N_{1}=17$ & $3(\mathrm{~S})$ \\
ri cui pero & $3(\mathrm{~S})$
\end{tabular}

Stazioni senza incertezze iniziali:

$$
N_{2}=11
$$

Magn: $4,4-4,6$

$N_{2} / N_{1}=64,7 \%$ (percentuale delle stazioni valide in partenza)

Il controllo è stato eseguito per la dromocrona relativa alla seguente profondità

$$
h=17,5 \mathrm{~km}
$$

Osservazion i ge nerali. Le 17 stazioni considerate coprono quasi con continuità l'intervallo delle $A$ lino a $20^{\circ}$ (c'è un vuoto tra $7^{\circ}$ e 110) peró il terremoto dà luogo a perplessità, specie se si considera l'SD. Anche se $1 \%$ Isc è clata senza correzione, e l'errore di $H$ è piuttosto piccolo, pure è da tener presente che il tempo $H$ di Atene differisce da quello dell'ISC di 2 sec. (v. tab. n. 1) e Atene si trova a soli $410 \mathrm{~km}$ dall'epicentro. Inoltre ci sono residui piuttosto alti (v. tab. n. 2). 
In ogni modo il nostro controllo lo faremo, per nniformitì, con l'Isc, all'mica profonditi possibile $(h=17,5 \mathrm{~km})$ ricordando ehe per questa dromocrona esistono solo le $P_{n}$ generali.

La I serie dà i seguenti risultati:

$$
\left.\begin{array}{c}
h=17,5 \mathrm{~km} \\
\text { (con } N_{1}=17 \text { stazioni) }
\end{array} \begin{array}{l}
\mathrm{A}=23,5 \% \\
\mathrm{~B}=17,5 \% \\
\mathrm{C}=47,1 \% \\
\mathrm{I}=11,8 \%
\end{array}\right\}(\mathrm{A}+\mathrm{B})=41 \%
$$

e la II serie da:

$$
\left.\begin{array}{l}
h=17,5 \mathrm{~km} \\
N_{2}=11 \text { stazioni) }
\end{array} \quad \begin{array}{l}
\mathrm{A}=36,4 \% \\
\mathrm{~B}=9,1 \% \\
\mathrm{C}=45,5 \% \\
\mathrm{D}=9,1 \%
\end{array}\right\}(\mathrm{A}+\mathrm{B})=45,5 \%
$$

La II serie non migliora molto i risultati della $I$ : resta elevato il numero di stazioni assegnate alla classe c, e coe ancora ma stazione assegnata alla classe $\mathrm{D}$.

In questo caso, anche se esula dagli schemi fissati, abbiamo voluto calcolare la $I I I$ scrie ottenuta tenendo conto rei $\delta A$ dovuti al $\pm 0^{\text {s, }} 96$ dell $\left.H\right)$ e abbiamo ottenuto:

$$
\begin{array}{ll}
h=17,5 & \begin{array}{l}
\mathrm{A}=41,2 \% \\
\mathrm{~B}=23,5 \%
\end{array} \\
\begin{array}{l}
\mathrm{C}=29,4 \% \\
\mathrm{C} 17 \text { stazioni })
\end{array} & \left.\begin{array}{l}
\mathrm{C}=5,9 \% \\
\mathrm{~A}=45,5 \% \\
\mathrm{~B}=27,3 \%
\end{array}\right\}(\mathrm{A}+\mathrm{B})=64,7 \% \\
\text { (con } 11 \text { stazioni) } & \begin{array}{l}
\mathrm{C}=27,3 \% \\
\mathrm{C}=17,5 \%
\end{array}
\end{array}
$$

In questo morlo le cose migliorerebbero considerevolmente, anche se le $A$ non raggimgrono nemmeno il $50 \%$; peró le D sono scomparse.

Facciamo notare a questo punto, che in base allosservazione fatta sull $H$ di Atene, abbiamo eseguito il controllo anche con quel tempo origine: non riportiamo i risultati numerici, peró possiamo dire che con quell' $H$ le cose migliorerebbero molto, specialmente per le stazioni greche per le quali tutte le c diventerebbero $\Lambda$.

La $I V$ serie non si puo calcolare perché ei sono solo le $P_{n}$ generali.

R is $\mathrm{l}_{\mathrm{t}}$ a $\mathrm{t}$ i fi na $\mathrm{i}$. Dal controllo generale fatto non si puo dire che $l^{\prime} h=17,5 \mathrm{~km}$ confermi $l^{\prime} h=16 \mathrm{~km}$, in maniera soddisfa- 
cente: il numero dei $\mathrm{C}$ è ancora piuttosto elevato e gli $I$ sono meno del $50 \%$.

Le stazioni italiane non hamo registrato. Solo a lioma IIP compare una $P^{*}$.

Terr. n. 21) Creta.

$H=20^{\mathrm{n}} 18^{\mathrm{m}} 03^{\mathrm{s}}, 8$ 土 $0^{\mathrm{s}}, 66$

$\mathrm{SI})=1,61$ (su 136 osservazioni)

$h_{\mathrm{isc}}=(35 \pm 5,5) \mathrm{km}$

$7 \mathrm{vscgs}=53 \mathrm{~km}$

Magn: $\sim 5,1$

Stazioni prese in esame fino a $20^{\circ}$.

\begin{tabular}{l|lc}
$N_{\mathrm{I}}=37$ & $\tau(\dot{\mathrm{S}})$ \\
di cui però & $2(\dot{\mathrm{S}})$ \\
1 (S)
\end{tabular}

Stazioni senza incertezze iniziali:

$$
N_{\mathrm{s}}=27
$$

$N_{2} / N_{1}=73 \%$ (percentuale delle stazioni valide in partenza)

Il controllo è stato eseguito per le dromocrone relative alle soguenti profonditì

$$
\left\{\begin{array}{l}
h_{1}=35 \mathrm{~km} \leftarrow \\
h_{2}=50 \mathrm{~km}
\end{array}\right.
$$

Osservazioni generali. Le stazioni coprono con contimuità l'intervallo delle $A$ fino a $20^{\circ}$.

Esaminando i cati di partenza riassunti nella tab. n. 1 si nota man grancle diversita tra i lati dei vari osservatori. Mosea, Atene, l'USCGS e il BCIS calcolano $H$ e $h$ piuttosto discordanti tra loro e con l'ISC: (da segnalare $H_{\mathrm{sosca}}-H_{\mathrm{ISc}}=11 \mathrm{s,2}$ e $\left.h_{\text {xosca }}-h_{\mathrm{ISC}}=85 \mathrm{~km}\right)$.

Il nostro controllo, come al solito, si riferisce all'ISC.

La $I$ serie dà i risultati seguenti:

\begin{tabular}{|c|c|c|}
\hline$P_{n}(\operatorname{grn})$. & $I_{n_{1}}$ & $I_{n_{2}}^{\prime}$ \\
\hline $\begin{array}{l}A=59.5 \% \\
\mathrm{~B}=16,2 \% \\
\mathrm{C}=16,2 \% \\
\mathrm{~J}=8,1 \% \\
(\Lambda+B)=75,7 \%\end{array}$ & $\begin{array}{l}A=59,5 \% \\
B=16,2 \% \\
C=18,9 \% \\
I)=5,4 \% \\
(A \div B)=75,7 \%\end{array}$ & $\begin{array}{l}A=48,7 \% \\
B=24,3 \% \\
(C=8,1 \% \\
1)=18,9 \% \\
(A=B)=73 \%\end{array}$ \\
\hline
\end{tabular}

$$
\begin{aligned}
h_{1} & =35 \mathrm{~km} \\
\left(\operatorname{con} N_{1}\right. & =37 \text { stazioni })
\end{aligned}
$$




$$
\begin{aligned}
h_{2} & =50 \mathrm{~km} \\
\left(\operatorname{con} H_{1}\right. & =37 \text { stazioni) }
\end{aligned}
$$

\begin{tabular}{|c|c|c|}
\hline$I_{n}^{\prime}(\operatorname{gen})$. & $I^{\prime}{ }_{n_{1}}$ & $I_{12}$ \\
\hline $\begin{array}{l}A=54,1 \% \\
B=21,6 \% \\
r=18,0 \% \\
1)=5,4 \% \\
(A-B)=75,7 \%\end{array}$ & $\begin{array}{l}A=59,5 \% \\
B=16,2 \% \\
C=21,6 \% \\
B=2,7 \% \\
(A=B)=75,7 \%\end{array}$ & $\begin{array}{l}A=8,1 \% \\
B=8,1 \% \\
C=16,2 \% \\
D=67,6 \% \\
(A \therefore B)=16,2 \%\end{array}$ \\
\hline
\end{tabular}

La II serie dì:

$$
l_{1}=35 \mathrm{~km}
$$

\begin{tabular}{|c|c|c|}
\hline$P_{n}($ gen. $)$ & $I^{\prime}{ }_{11}$ & $I^{\prime}{ }_{12}$ \\
\hline $\begin{array}{l}A=77,8 \% \\
B=22,2 \% \\
(C=0 \\
D=0 \\
(A+B)=100 \%\end{array}$ & $\begin{array}{l}A=81,5 \% \\
B=14,8 \% \\
(1=3,7 \% \\
1)=0 \\
(A+B)=96,3 \%\end{array}$ & $\begin{array}{l}A=55,6 \% \\
B=33,3 \% \\
C=11,1 \% \\
1)=0 \\
(A-B)=89 \%\end{array}$ \\
\hline
\end{tabular}

(con $N_{2}-27$ stazioni)

\begin{tabular}{|c|c|c|}
\hline$P_{n}($ gen.) & $I_{n_{1}}$ & $I_{n_{2}}^{\prime}$ \\
\hline $\begin{array}{l}A=70,4 \% \\
B=22,2 \% \\
C=7,4 \% \\
1)=0 \\
(A+B)=92,6 \%\end{array}$ & $\begin{array}{l}A=66,7 \% \\
B=11,1 \% \\
(1=22,2 \% \\
D=0 \\
(A+B)=77,8 \%\end{array}$ & $\begin{array}{l}A=7,4 \% \\
B=7,4 \% \\
C=18,5 \% \\
D=66,7 \% \\
(A-B)=14,8 \%\end{array}$ \\
\hline
\end{tabular}

$h_{\mathrm{2}}=50 \mathrm{~km}$

(con $N_{2}-27$ stazioni)

Questa serie darebbe una preferenta ai risultati ottenuti per $h=35 \mathrm{~km}$ (specialmente nelle $P_{n}$ (generali)), i $O$ e i 10 sono tutti scomparsi.

Lat $I I I$ serie non è stata ealcolata perché l'errore di $H$ è piecolo $( \pm 0 \mathrm{~s}, 66)$. 
Lal $I V$ serie (le $P_{n}$ sono o $P_{n 1}$ o $P_{n 2}$ ) dà:

$$
\begin{aligned}
& \begin{array}{c|}
h_{1}=35 \mathrm{~km} \\
\text { (con } 37 \text { stazioni) }
\end{array} \quad \begin{array}{l}
A=75,7 \% \\
B=5,4 \% \\
C=13,5 \% \\
D=5,4 \%
\end{array} \\
& \begin{array}{c}
h_{1}=35 \mathrm{~km} \\
\text { (con } 27 \text { stazioni) }
\end{array} \quad \begin{array}{l}
A=92,6 \% \\
\mathrm{~B}=7,4 \% \\
\mathrm{C}=0 \\
\mathrm{D}=0
\end{array} \\
& \begin{array}{c|l}
h_{2}=50 \mathrm{~km} & \begin{array}{l}
\mathrm{A}=67,6 \% \\
\mathrm{~B}=24,3 \% \\
\text { (con } 37 \text { stazioni) }
\end{array} \\
\mathrm{C}=8,1 \% \\
\mathrm{D}=0
\end{array} \\
& \left.\left.7_{2}=50 \mathrm{~km} \quad \begin{array}{l}
\mathrm{A}=74,1 \% \\
\mathrm{~B}=18,5 \%
\end{array}\right\} \cdot \mathrm{A}+\mathrm{B}\right)=92,6 \% \\
& \begin{array}{l|l}
\text { (con } 27 \text { stazioni) } & \begin{array}{l}
\mathrm{D}=7,4 \% \\
\mathrm{D}=0
\end{array}
\end{array}
\end{aligned}
$$

R i s u ltati fin a li. I migliori risultati si hamno senz'altro per $h=35 \mathrm{~km}$, migliorando ad ogni serie successiva ed aumentando la divergenza rispetto all'altra profondità. Nella IV serie si raggiunge il risultato migliore con le 27 stazioni valide, i C e $\mathrm{D}$ scompaiono e le A raggiungono il $93 \%$.

Le stazioni italiane hamno registrato solo delle tracce occultate dalla agitazione microsismica. La sola Trieste da ma $P_{n}$, ma con un forte scarto: $(\mathrm{O}-\mathrm{C})=-4^{\mathrm{s}}$.

Terr. n. 22) Creta

$$
\begin{aligned}
& H=15^{\mathrm{n}} 48^{\mathrm{m}} 31^{\mathrm{s}} \pm 1^{\mathrm{s}}, 1 \\
& \mathrm{SD}=3,09(\mathrm{su} 56 \text { osservazioni })
\end{aligned}
$$

$h_{\mathrm{ISc}}=(43 \pm 11) \mathrm{km}$

Magn: $4,1-5,1$

Stazioni prese in esame fino a $20^{\circ}$.

\begin{tabular}{l|lc}
$N_{1}=19$ & 5 & $(\dot{\mathrm{S}})$ \\
di cui però & $\tilde{j}$ & $(\ddot{\mathrm{S}})$ \\
1 & $(\dot{\mathrm{S}})$
\end{tabular}

Stazioni senza incertezze iniziali:

$$
N_{2}=8
$$

$N_{2} / N_{1}=42,1 \%$ o (percentuale delle stazioni valicle in partenza) 
Il controllo ì stato eseguito per le dromocrone relative alle seguenti profoncitit

$$
\left\{\begin{array}{l}
h_{1}=35 \mathrm{~km} \\
h_{0}=45 \mathrm{~km}
\end{array}\right.
$$

Osservazion i generali. I lati di partenza sono pinttosto incerti: in particolare si nota ma grande diversiti negli $H$ dei vari centri (p. es. $H_{\mathrm{Isc}}-H_{\mathrm{BCIS}}=6^{\mathrm{s}}$ ); anche l'SD ̀̀ alto; ed ̀̀ bassa la percentuale delle stazioni valide in partenza. Le 1 delle stazioni fino a $20^{\circ}$ presentano un vuoto nell'intervallo $10^{\circ}-16^{\circ}$.

I risultati della nostra indagine somo i seguenti:

La I serie dà:

\begin{tabular}{|c|c|c|}
\hline$P_{n}($ gren. $)$ & $I_{n_{1}}$ & $P_{n_{2}}$ \\
\hline $\begin{array}{l}A=21,1 \% \\
B=26,3 \% \\
C=26,3 \% \\
D=26,3 \% \\
(A+B)=47,4 \%\end{array}$ & $\begin{array}{l}A=31,6 \% \\
B=21,1 \% \\
C=21,1 \% \\
1)=26,3 \% \\
(A+B)=52,7 \%\end{array}$ & $\begin{array}{l}A=21,1 \% \\
13=10,5 \% \\
C=47,4 \% \\
D=21,1 \% \\
(A+13)=31,6 \%\end{array}$ \\
\hline
\end{tabular}

$$
\begin{gathered}
h_{1}=35 \mathrm{~km} \\
\left(\operatorname{con} N_{1}=19\right. \text { stazioni) }
\end{gathered}
$$

\begin{tabular}{|c|c|c|}
\hline$I_{n}(\operatorname{grc} 11)$. & $P_{n_{1}}$ & $I_{n_{2}}^{\prime}$ \\
\hline $\begin{array}{l}A=31,6 \% \\
B=21,1 \% \\
C=21,1 \% \\
1)=26,3 \% \\
(A+B)=52,7 \%\end{array}$ & $\begin{array}{l}A=26,3 \% \\
B=21,1 \% \\
C=21,1 \% \\
B=31,6 \% \\
(A+B)=47,4 \%\end{array}$ & $\begin{array}{l}A=21,1 \% \\
B=26,3 \% \\
C=21,1 \% \\
D=31,6 \% \\
(A+B)=47,4 \%\end{array}$ \\
\hline
\end{tabular}

$$
\begin{gathered}
h_{2}=45 \mathrm{~km} \\
\left(\bullet \circ N_{1}=19\right. \text { stazioni) }
\end{gathered}
$$

Le rlue $h$ sembrano egualmente probabili; i valori di c e 1 sono molto alti.

La II serie darà certamente risultati migliori (però si noti che sono state scartate più del $50 \%$ delle stazioni): 


$$
\begin{gathered}
h_{1}=35 \text { kinl } \\
(\text { con } 8 \text { stazioni) }
\end{gathered}
$$

\begin{tabular}{|c|c|c|}
\hline$P_{n}(g 011)$. & $I_{n_{1}}$ & $I_{n_{2}}$ \\
\hline $\begin{array}{l}\mathrm{A}=50,0 \% \\
\mathrm{~B}=50,0 \% \\
\mathrm{C}=0 \\
\mathrm{I}=0 \\
(\mathrm{~A}+\mathrm{B})=100 \%\end{array}$ & $\begin{array}{l}A=75,0 \% \\
B=25,0 \% \\
C^{\prime}=0 \\
1)=0 \\
(A+B)=100^{\circ}\end{array}$ & $\begin{array}{l}A=37,5 \% \\
B=12,5 \% \\
C=50,0 \% \\
1)=0 \\
(A+B)=50 \%\end{array}$ \\
\hline
\end{tabular}

\begin{tabular}{|c|c|c|}
\hline$P_{n}$ (gen.) & $P_{n_{1}}$ & $P_{n_{2}}$ \\
\hline $\begin{array}{l}A=75,0 \% \\
B=25,0 \% \\
C=0 \\
D=0 \\
(A+B)=100 \%\end{array}$ & $\begin{array}{l}A=50,0 \% \\
B=12,5 \% \\
C=37,5 \% \\
B=0 \\
(A+B)=62,5 \%\end{array}$ & $\begin{array}{l}\Lambda=50,0 \% \\
13=37,5 \% \\
(\vdots=12,5 \% \\
1)=0 \\
(A+B)=87,5 \%\end{array}$ \\
\hline
\end{tabular}

$$
\begin{gathered}
h_{2}=45 \mathrm{~km} \\
(\operatorname{con} 8 \text { stazioni })
\end{gathered}
$$

Da entrambe le dromocrone sono scomparsi tutti i D e alcuni C. I risultati migliori si raggiungono con la $P_{n 1}$ per i $35 \mathrm{~km}$ e con la $P_{n}$ (generale) per $45 \mathrm{~km}$ dove gli $A$ diventano il $75,0 \%$. Però, ripetiamo, otto stazioni sono poche per poterne trarre conclusioni attendibili.

La III serie, ove si calcolasse, (tenendo conto del $\pm 1^{\text {s, }} 1$ (lell' $H$ ) comporterebbe delle oscillazioni, sulle $A$, dell'ordine dei $\pm 9 \mathrm{~km}$; si avrebbe un pò di miglioramento, ma resterebbero sempre grosse incertezze.

Meglio invece la $I V$ serie calcolata tenendo presente che le $P_{n}$ sono o $P_{n 1}$ o $P_{n 2}$ : essa dà:

$$
\begin{array}{cc}
h_{1}=35 \mathrm{~km} & \begin{array}{l}
\mathrm{A}=42,1 \% \\
\mathrm{~B}=15,8 \% \\
\mathrm{C}=36,8 \% \\
\mathrm{D}=5,3 \%
\end{array} \\
h_{1}=35 \mathrm{~km} & \begin{array}{l}
\mathrm{A}=87,5 \% \\
\mathrm{~B}=12,5 \% \\
\text { (con } N_{1}=19 \text { stazioni) }
\end{array} \\
N_{2}=8 \text { stazioni) } & \begin{array}{l}
\mathrm{C}=0 \\
\mathrm{D}=0
\end{array}
\end{array}
$$




$$
\begin{aligned}
& \begin{array}{l}
h_{2}=45 \mathrm{~km} \\
\Lambda_{\mathrm{I}}=19 \text { stazioni) }
\end{array}\left\{\begin{array}{l}
\Lambda=47,4 \% \\
\mathrm{~B}=21,1 \% \\
\mathrm{C}=15,8 \% \\
\mathrm{D}=15,8 \%
\end{array}\right\}(\mathrm{A}+\mathrm{B})=68,5 \% \\
& \left.h_{2}=45 \mathrm{~km} \quad \begin{array}{l}
\Lambda=100 \% \\
\mathrm{~B}=0
\end{array}\right\}(A+\mathrm{B})=100 \% \\
& \text { (con } N_{2}=8 \text { stazioni) } \mid \begin{array}{l}
\mathrm{C}=0 \\
\mathrm{D}=0
\end{array}
\end{aligned}
$$

Ris lu lati finali. La IV serie conferma, migliorandoli $(\Lambda=100 \%)$, i risultati della II serie, per cui entrambe le $h$ sono possibili: tuttavia permane, in tutta l'analisi, lo stato di grave incertezar dovuto alle discordanze di partenza.

Le stazion i it a liane hamno registrato il terremoto solo come traccia.

Terr. n. 23) Rodi

$H=20^{\mathrm{h}} 58^{\mathrm{m}} 11^{\mathrm{s}}, 9 \pm 0^{\mathrm{s}}, 47$

$\mathrm{SD}=1,67$ (su 162 osservazioni)

$h_{\text {Isc }}=(35 \pm 4,7) \mathrm{km}$

$h_{\mathrm{USCGS}}=43 \mathrm{~km}$

Magn: $4,7-5,0$
Stazioni prese in esame fino a $20^{\circ}$.

\begin{tabular}{l|lc}
$N_{1}=51$ & & $(\dot{\mathrm{S}})$ \\
di cui però & $\pm(\ddot{\mathrm{S}})$ \\
1 & $(\dot{\mathrm{S}})$
\end{tabular}

Stazioni senza incertezze iniziali:

$$
N_{2}=39
$$

$N_{2} / N_{1}=76,5 \%$ (percentuale delle stazioni valicle in partenza)

Il controllo è stato eseguito per le dromocrone relative alle seguenti profonditì

$$
\left\{\begin{array}{l}
h_{1}=35 \mathrm{~km} \leftarrow \\
h_{2}=40 \mathrm{~km} \leftarrow
\end{array}\right.
$$

nonostante che la loro differenza sia poco significativa.

Osservazioni generali. Il terremoto ha dati di partenza degni di fiducia. Si prevede un controllo positivo. Le numerose stazioni coprono con continuità le $A$ fino a $20^{\circ}$.

La $I$ serie dà i seguenti risultati (con $N_{1}$ stazioni): 
$h_{1}=35 \mathrm{~km}$
$(\operatorname{con} 51$ stazioni)

\begin{tabular}{|l|l|l|}
\hline \multicolumn{1}{|c|}{$P_{n}$ (gen.) } & \multicolumn{1}{|c|}{$P_{n_{1}}$} & \multicolumn{1}{c|}{$P_{n_{2}}$} \\
\hline & & \\
$\mathrm{A}=64,7 \%$ & $\mathrm{~A}=62,8 \%$ & $\mathrm{~A}=41,2 \%$ \\
$\mathrm{~B}=11,8 \%$ & $\mathrm{~B}=17,6 \%$ & $\mathrm{~B}=23,5 \%$ \\
$\mathrm{C}=13,7 \%$ & $\mathrm{C}=13,7 \%$ & $\mathrm{C}=21,6 \%$ \\
$\mathrm{I}=9,8 \%$ & $\mathrm{D}=5,9 \%$ & $\mathrm{~J}=13,7 \%$ \\
$(\mathrm{~A}+\mathrm{B})=76,5 \%$ & $(\mathrm{~A}+\mathrm{B})=80,4 \%$ & $(\mathrm{~A}+\mathrm{B})=64,7 \%$ \\
\hline
\end{tabular}

$h_{2}=40 \mathrm{~km}$

(con 5l stazioni)

\begin{tabular}{|c|c|c|}
\hline$P_{n}(g(n)$. & $P_{n_{1}}$ & $P_{n 2}^{\prime}$ \\
\hline $\begin{array}{l}A=70,6 \% \\
B=9,8 \% \\
C=11,8 \% \\
D=7,8 \% \\
(A+B)=80,4 \%\end{array}$ & $\begin{array}{l}\mathrm{A}=51 \% \\
\mathrm{~B}=19,6 \% \\
\mathrm{C}=19,6 \% \\
\mathrm{D}=9,8 \% \\
(\mathrm{~A}+\mathrm{B})=70,6 \%\end{array}$ & $\begin{array}{l}A=58.8 \% \\
B=13,7 \% \\
C=17,6 \% \\
D=9,8 \% \\
(A+B)=72,5 \%\end{array}$ \\
\hline
\end{tabular}

Le rromocrone controllate relative alle due $h$ sembrano dare entrambe risultati egualmente attendibili come era preverlibile.

La II serie dà i seguenti risultati (con $\mathrm{N}_{2}$ stazioni)

$$
\begin{gathered}
h_{1}=35 \mathrm{~km} \\
(\operatorname{con} 39 \text { stazioni) }
\end{gathered}
$$

\begin{tabular}{|l|l|l|}
\hline \multicolumn{1}{|c|}{$P_{n}$ (gen.) } & \multicolumn{1}{|c|}{$P_{n_{1}}$} & \multicolumn{1}{c|}{$P_{n_{2}}$} \\
\hline & & \\
$\mathrm{A}=84,6 \%$ & $\mathrm{~A}=82,0 \%$ & $\mathrm{~A}=51,3 \%$ \\
$\mathrm{~B}=7,7 \%$ & $\mathrm{~B}=15,4 \%$ & $\mathrm{~B}=28,2 \%$ \\
$\mathrm{C}=7,7 \%$ & $\mathrm{C}=2,6 \%$ & $\mathrm{C}=17,9 \%$ \\
$\mathrm{D}=0$ & $\mathrm{D}=0$ & $\mathrm{D}=2,6 \%$ \\
$(\mathrm{~A}+\mathrm{B})=92,3 \%$ & $(\mathrm{~A}+\mathrm{B})=97,4 \%$ & $(\mathrm{~A}+\mathrm{B})=79,5 \%$ \\
\hline
\end{tabular}


$h_{2}=40 \mathrm{~km}$

(ron 39 stazioni)

\begin{tabular}{|l|l|l|}
\hline$P_{n}$ (gen.) & \multicolumn{1}{|c|}{$P_{{ }_{11}}$} & \multicolumn{1}{c|}{$P_{n_{2}}$} \\
\hline & $\mathrm{A}=61,5 \%$ & $\mathrm{~A}=76,9 \%$ \\
$\mathrm{~A}=89,7 \%$ & $\mathrm{~B}=20,5 \%$ & $\mathrm{~B}=15,4 \%$ \\
$\mathrm{C}=5,1 \%$ & $\mathrm{C}=7,7 \%$ \\
$\mathrm{D}=0$ & $\mathrm{C}=17,9 \%$ & $\mathrm{D}=0$ \\
$(\mathrm{~A}+\mathrm{B})=94,8 \%$ & $(\mathrm{~A}+\mathrm{B})=82 \%$ & $(\mathrm{~A}+\mathrm{B})=92,3 \%$ \\
\hline
\end{tabular}

Questa serie conferma, migliorandoli, i risultati ciella I serie.

La $I I I$ serie è inutile calcolarla perché $\delta H= \pm 0$ s, \pm 7 .

La $I V$ serie (le $P_{n}$ sono o $P_{n 1}$ o $P_{n 2}$ ) dà:

$$
\begin{aligned}
& \left.\begin{array}{l}
h_{1}=35 \mathrm{~km} \\
\text { con } N_{1} \text { stazioni) }
\end{array} \begin{array}{l}
\mathrm{A}=72,6 \% \\
\mathrm{~B}=11,8 \% \\
\mathrm{C}=11,8 \% \\
\mathrm{D}=3,9 \%
\end{array}\right\}(\mathrm{A}+\mathrm{B})=84,4 \%
\end{aligned}
$$

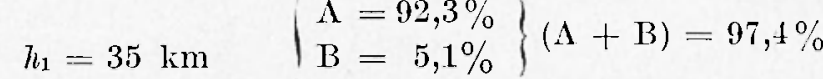

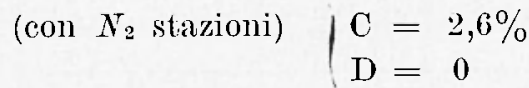

$$
\begin{aligned}
& \begin{array}{c}
h_{2}=40 \mathrm{~km} \\
\left(\text { con } N_{1} \text { stazioni }\right)
\end{array}\left\{\begin{array}{l}
\mathrm{A}=74,5 \% \\
\mathrm{~B}=9,8 \% \\
\mathrm{C}=11,8 \% \\
\mathrm{D}=3,9 \%
\end{array}\right\}(\mathrm{A}+\mathrm{B})=81,3 \% \\
& \left.\begin{array}{c}
h_{2}=40 \mathrm{~km} \\
\text { con } N_{2} \text { stazioni) }
\end{array} \begin{array}{l}
A=92,3 \% \\
\mathrm{~B}=5,1 \% \\
\mathrm{C}=2,6 \% \\
\mathrm{D}=0
\end{array}\right\}(\Lambda+\mathrm{B})=97,4 \%
\end{aligned}
$$

Anche secondo questa serie ie ane profondita sono entramibe valide e probabili. Naturalmente i risultati migliori sono sempre quelli in cui non intervengono le stazioni con incertezze iniziali.

R is ultati f in a li. L'analisi conferma le previsioni. Te due dromocrone controllate relative alle due $h$ sono entrambe ottimamente valide. I controlli fatti, pur conservando la situazione delineatasi 
inizialmente, costituiscono un successivo miglioramento nelle percentuali.

Le stazioni italiane hamo registrato quasi tutte: peró a Roma MP le $P_{n}$ arrivano con un po di anticipo, e troverebbero la loro interpretazione migliore come $P_{n \mathrm{I}}$ per $h=40 \mathrm{~km}$. Invece le prime onde registrate a Padova sono indubbiamente successive alle $I_{n}$ (v. tab. residui $(\mathrm{O}-\mathrm{C})=+7 \mathrm{~s}, 1)$.

'Terr. n. 24) Turchia

$H=17^{\mathrm{h}} 45^{\mathrm{m}} 57^{\mathrm{s}}, 0 \mathrm{1}^{\mathrm{s}} 0^{\mathrm{s}}, 14$

$\mathrm{SD}=1,44$ (su 123 osservazioni $)$

$h_{1 \mathrm{sc}}=(\tilde{\mathrm{\jmath}} 9 \pm 4, \tilde{5}) \mathrm{km}$

$h_{\mathrm{USCGS}}=40 \mathrm{~km}$

Magn: 5,6
Stazioni prese in esame fino a $20^{\circ}$. $N_{1}=30$ ali cui però 10

(S) (per $h_{1}=40 \mathrm{~km}$ )

$N_{1}=25$ ai cui però 10

(S) (per $h_{2}:=60 \mathrm{~km}$ )

Stazioni senza incertezze iniziali:

$$
\lambda_{2}=20 \text { (per } h_{1}=40 \mathrm{~km} \text { ) }
$$$$
N_{2}=1 \tilde{5} \text { (per } h_{2}=60 \mathrm{~km} \text { ) }
$$

$\lambda_{2} / N_{1}=66,7 \%$ (per $\left.h_{1}=40 \mathrm{~km}\right)$ $60 \% \quad\left(1017_{2}=60 \mathrm{~km}\right)$

(percentuali delle stazioni valide in partenza)

Il controllo è stato eseguito per le dromocrone relative alle seguenti profondita

$$
\left\{\begin{array}{l}
h_{1}=40 \mathrm{~km} \\
h_{0}=60 \mathrm{~km} \leftarrow
\end{array}\right.
$$

Osservazioni generali. Le stazioni che hamno registrato si trovano a distanze che coprono con continuita l'intervallo $A$ fino a $20^{\circ}$.

Come al solito i nostri controlli vengono eftettuati riferendoci all'ISC, però è da notare la diversità degli $H$ degli altri centri (per es. $H_{\mathrm{Isc}}-H_{\text {Mosea }}=4^{\mathrm{s}}$ ).

Abbiamo preso in esame le due $h$ date dall'ISC e dall'USCGS; facciamo notare che nel controllo per $h=60 \mathrm{~km}$ abbiamo dovuto sopprimere le כ̃ stazioni con $A>18^{\circ}$ perché la dromocrona $h=60 \mathrm{~km}$ da noi calcolata non arriva fino a quelle distanze $\left(^{3}\right)$ e una sua estarapolazione sarebbe stata arbitraria. 
I risultati dell'esame sono $\mathrm{i}$ seguenti:

Isa I serie dia:

$$
h_{1}=40 \mathrm{~km}
$$

(con $N_{1}=30$ stazioni)

\begin{tabular}{|c|c|c|}
\hline$I_{n}^{\prime}($ gen. $)$ & $P_{n_{1}}$ & $P_{n_{2}}$ \\
\hline $\begin{array}{l}A=46,7 \% \\
B=23,3 \% \\
C=26,7 \% \\
D=3,3 \% \\
(A+B)=70 \%\end{array}$ & $\begin{array}{l}A=46,7 \% \\
B=23,3 \% \\
C=26,7 \% \\
D=3,3 \% \\
(A+B)=70 \%\end{array}$ & $\begin{array}{l}A=36,7 \% \\
\mathrm{~B}=23,3 \% \\
\mathrm{C}=26,7 \% \\
\mathrm{~B}=13,3 \% \\
(\mathrm{~A}+\mathrm{B})=60 \%\end{array}$ \\
\hline
\end{tabular}

$$
h_{2}=60 \mathrm{~km}
$$

(con $N_{1}=25$ stazioni)

\begin{tabular}{|l|l|l|}
\hline \multicolumn{1}{|c|}{$P_{n}$ (gen.) } & \multicolumn{1}{|c|}{$P_{n_{1}}$} & $P_{n_{2}}$ \\
\hline & & \\
$\mathrm{A}=52,0 \%$ & $\mathrm{~A}=56,0 \%$ & $\mathrm{~A}=44 \%$ \\
$\mathrm{~B}=16 \%$ & $\mathrm{~B}=24 \%$ & $\mathrm{~B}=24 \%$ \\
$\mathrm{C}=32 \%$ & $\mathrm{C}=16 \%$ & $\mathrm{D}=28 \%$ \\
$\mathrm{D}=0 \%$ & $\mathrm{D}=4 \% \%$ & $(\mathrm{~A}+\mathrm{B})=68 \%$ \\
$(\mathrm{~A}-\mathrm{B})=68 \%$ & $(\mathrm{~B}) \mathrm{B})=80 \%$ & \\
\hline
\end{tabular}

Si alelineano migliori risultati per $h=60 \mathrm{~km}$.

Isa $I I$ serie conferma la tendenza delineatasi. Infatti:

\begin{tabular}{|c|c|c|}
\hline$P_{n}($ gen. $)$ & $P_{n_{1}}$ & $P_{n 2}$ \\
\hline $\begin{array}{l}A=70 \% \\
B=25 \% \\
C=5 \% \\
1=0 \\
(A+B)=95 \%\end{array}$ & $\begin{array}{l}A=65 \% \\
B=15 \% \\
C=15 \% \\
D=5 \% \\
(A+B)=80 \%\end{array}$ & $\begin{array}{l}A=45 \% \\
B=20 \% \\
C=35 \% \\
D=0 \\
(A+B)=65 \%\end{array}$ \\
\hline
\end{tabular}

$$
\begin{aligned}
h_{1} & =40 \mathrm{~km} \\
\left(\operatorname{con} N_{2}\right. & =20 \text { stazioni })
\end{aligned}
$$


$h_{2}=60 \mathrm{~km}$

(con $N_{2}=15$ stazioni)

\begin{tabular}{|l|l|l|}
\hline \multicolumn{1}{|c|}{$P_{n \text { (gen.) }}$} & \multicolumn{1}{|c|}{$P_{n_{1}}$} & \multicolumn{1}{c|}{$P_{n_{2}}$} \\
\hline $\mathrm{A}=80 \%$ & $\mathrm{~A}=73,3 \%$ & $\mathrm{~A}=60 \%$ \\
$\mathrm{~B}=13,3 \%$ & $\mathrm{~B}=20 \%$ & $\mathrm{~B}=26,7 \%$ \\
$\mathrm{C}=6,7 \%$ & $\mathrm{C}=6,7 \%$ & $\mathrm{C}=13,3 \%$ \\
$\mathrm{D}=0$ & $\mathrm{D}=0$ & $\mathrm{D}=0$ \\
$(\mathrm{~A}+\mathrm{B})=93,3 \%$ & $(\mathrm{~A} \div \mathrm{B})=93,3 \%$ & $(\mathrm{~A}+\mathrm{B})=86,7 \%$ \\
\hline
\end{tabular}

$\mathrm{L}^{\prime}(A+\mathrm{B})=95 \%$ delle $P_{n}$ (gen.) porterebbe una preferenza per $1^{\prime} h_{1}=40 \mathrm{~km}$, però per $h_{2}=60 \mathrm{~km}$ il numero delle $A \dot{e}$ superiore in tutti e tre i tipi di $P_{n}$, sicché è da preferire l' $h_{2}=60 \mathrm{~km}$.

La III serie non è stata calcolata. La correzione di $I I$ ̀̀ $+0^{\mathrm{s}, 11}$ e non porterebbe variazioni.

La $I V$ serie (dove le $P_{n}$ sono o $P_{n 1}$ o $P_{n 2}$ ) dà:

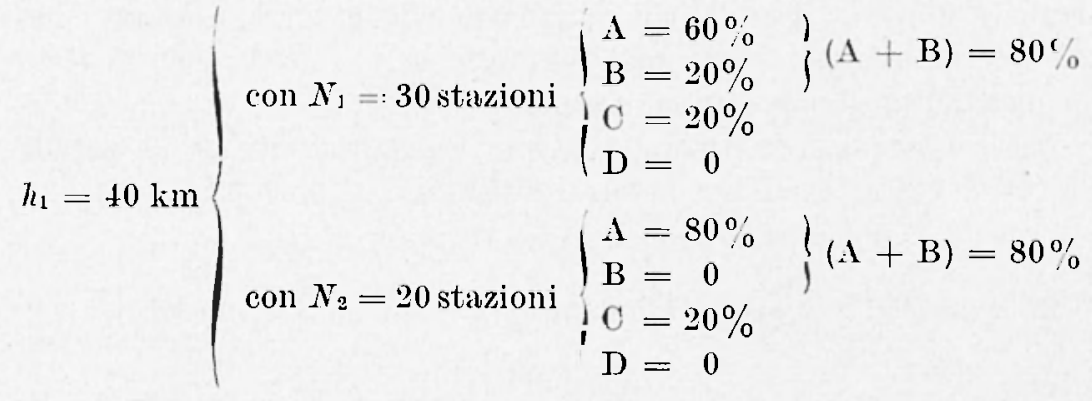

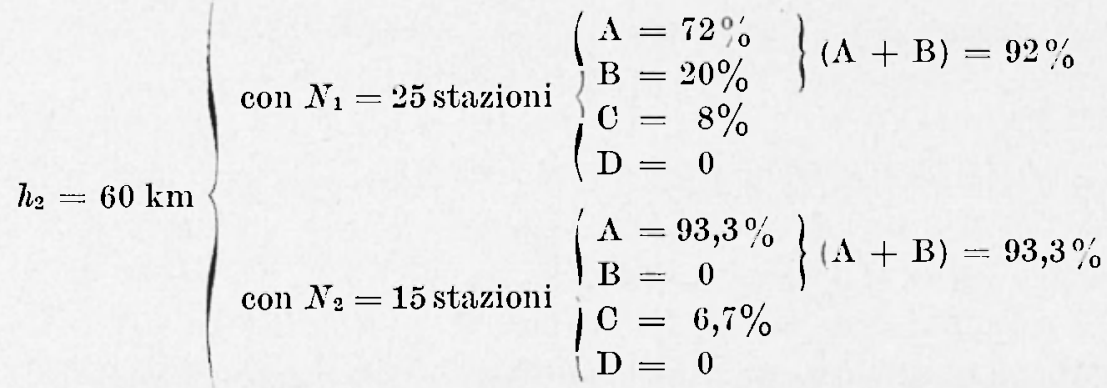

Il $\mathrm{r}$ is ultato mig I i re si ottiene dunque per $h_{2}=60 \mathrm{~km}$.

Le stazion i it a 1 i a ne hamno registrato solo parzialmente questo terremoto, e con residui piuttosto rilevanti. Solo Trieste si puó assegnare alla classe $\Lambda$. 
Terr. 11. 25) Egitto

$H=05^{11} 10^{\mathrm{m}}\left(03^{\mathrm{s}} \pm 2 \mathrm{2s}, 5\right.$

$\mathrm{SD}=1,21$ (su 57 osservazioni)

$h_{\text {Isc }}=(15 \pm 15) \mathrm{km}$

$\eta_{\text {USCGS }}=16 \mathrm{~km}$

Nagn: 4,8

Stazioni prese in esame fino a $20^{\circ}$.

\begin{tabular}{l|ll}
$N_{1}=12$ & 1 (ì) \\
di cui però & 1 (
\end{tabular}

Stazioni senza incertezze iniziali: $N_{2}=10$

$N_{2} / N_{1}=83,3 \%$ (percentuale delle stazioni valide in partenza)

Il controllo è stato eseguito per la dromocrona relativa alla seguente profonditì

$$
h=17,5 \mathrm{~km} \leftarrow
$$

Osservazioni generali. I dati di partenza destano perplessità. I'SI d̀ piccolo, però l'errore dell' $H$ ì forte ed anche la correzione dell'h sis. Inoltre e'c da notare che le $\Delta$ considerate fino a $20^{\circ}$ presentano un vuoto nell'intervallo $4^{\circ}, 4-13^{\circ}, 2$ : per il resto sono ristribuite abbastanzal uniformemente.

Isa dromocrona di controllo è solo quella per $h=17,5$ perché anche l'USCGS dà per $h$ un valore prossimo.

I risultati dellanalisi sono i seguenti:

Ia $I$ serie dia: (questa dromocrona ha solo le $P_{n}$ (generali)).

$$
\begin{array}{c|l}
h=17,5 \mathrm{~km} & \begin{array}{l}
\Lambda=50 \% \\
\mathrm{~B}=8,3 \%
\end{array} \\
\text { (con } N_{1}=12 \text { stazioni) } & \begin{array}{l}
\mathrm{C}=33,3 \% \\
\mathrm{D}=8,3 \%
\end{array}
\end{array}
$$

Ial $I I$ serie migliora orviamente i risultati

$$
\begin{aligned}
& \left.h=17, \tilde{\pi} \mathrm{km} \quad \begin{array}{l}
\mathrm{A}=60 \% \\
\mathrm{~B}=10 \%
\end{array}\right\}(\mathrm{A}+\mathrm{B})=70 \% \\
& \begin{array}{l|l}
\text { (con } N_{2}=10 \text { stazioni) } & \begin{array}{l}
\mathrm{C}=20 \% \\
\mathrm{D}=10 \%
\end{array}
\end{array}
\end{aligned}
$$

però ancora il peso dei $O$ e dei D è rilevante.

Ia III serie, ottenuta tenendo conto della forte correzione di $H( \pm 2,5)$ porta ai seguenti risultati, nettamente migliori: 


$$
\begin{aligned}
& \left.h=17,5 \mathrm{~km} \quad \begin{array}{l}
A=83,3 \% \\
B=16,7 \%
\end{array}\right\}(A+B)=100 \% \\
& \text { (con } N_{1}=12 \text { stazioni) } \quad \begin{array}{l}
\mathrm{C}=0 \\
\mathrm{D}=0
\end{array} \\
& \begin{array}{l}
h=17,5 \mathrm{~km} \\
N_{2}=10 \text { stazioni) }
\end{array}\left\{\begin{array}{l}
\mathrm{A}=90 \% \\
\mathrm{~B}=10 \% \\
\mathrm{C}=0 \\
\mathrm{D}=0
\end{array}\right\}(\mathrm{A}+\mathrm{B})=100 \%
\end{aligned}
$$

Isa $I V$ serie (le $P_{n}$ sono o $P_{n 1}$ o $P_{n 2}$ ) non si calcola perché ci sono solo le $P_{n}$ (generali) (essa equivale alla serie I e II).

$R$ is ultati fi nali. I risultati del controllo migliorano come al solito via via che si procede nelle serie.

Nella III serie si riesce a raggiungere il $90 \%$ degli $A$ tenendo conto dell'errore di $H$. Questo convalida l'importanza che assumono gli errori iniziali, su qualunque tipo di controllo.

Ise stazioni italiane non hamno registrato il sisma.

\section{DISCUSSIONE SUI RISULTATI DELL'INDAGINE SVOLTA E CONCLUSIONI}

Dalle analisi svolte risulta abbastanza chiaramente come il procedimento di controllo da noi usato si possa ritenere valido: esso conduce a risultati che vanno progressivamente migliomando da ma serie alla successiva.

La $I$ serie (fatta con tutte le $N_{1}$ stazioni riportate dal bollettino ISC fino a $A=20^{\circ}$ ) rispecchia sempre le incertezze iniziali più o meno gravi a seconcla del numero delle stazioni incerte $(\dot{\mathrm{S}}, \ddot{\mathrm{S}}, \dot{\overrightarrow{\mathrm{S}}})$, del valore dell'SD, e dei valori del $|\delta H|$ e tel $\mid \delta 7$ |.

Isa II serie (calcolata sulle $N_{2}$ stazioni che fino a $20^{\circ}$ presentano residui $(\mathrm{O}-\mathrm{C})<|2 \mathrm{~s}, \pm|)$ migliora sensibilmente le percentuali delle $\mathrm{A}$ e $\mathrm{B}$; le $\mathrm{C}$ si riducono e talvolta si annullano, le D sono quasi sempre assenti.

Ia III serie (calcolata quando lo esige un forte $\delta H \mid$ ) fa scomparire le gravi discordanze della I serie e talvolta migliora anche i risultati della II serie (v. ter'. n. 8).

Isa $I V$ serie infine (calcolata per tutte le $h-$ ad eccezione di $h=5 \mathrm{~km}$ e $h=17,5 \mathrm{~km}$ - ammettendo che le $P_{n}$ possano essere o $P_{n 1}$ 
o $P_{n 2}$ l'una escludendo l'altra) ha dato quasi sempre i risultati migliori. In quasi tutti i terremoti esaminati questa serie presenta

$$
(A+B)=100 \%(\operatorname{con} \mathrm{C}=0 \text { e } \mathrm{I})=0)
$$

e molto spesso addirittura

$$
A=100 \% \text { (essendo anche } B=0 \text { ). }
$$

Ijanalisi condotta in questo modo (i ha consentito altresì la seelta tra due profondità ipocentruli, là dove esisteva una ambiguità di partenza; in qualche caso (terr. 22 e 23) la scelta non è stata possibile perehé il controllo convalidava entrambe le $h$ con risultati $\Lambda$ e $(A+B)$ uguali, ma quando questo si è verificato le due $h$ in discussione differivano solo di $5-10 \mathrm{~km}$.

Nella tab. n. 1 sono riassunti in forma sintetica i risultati della ricerca relativi alle IV serie: per le profondità $5 \mathrm{~km}$ e $17,5 \mathrm{~km}$ le IV serie coincidono, come detto, con le I e le II (*).

Nella fig. 4 abbiamo tentato una rappresentazione grafica dei risultati dell'indagine svolta. Per ogni terremoto analizzato sono state riportate, nella successione geografica W-F, nella parte superiore del grafico, le profondità ipocentrali controllate evidenziando con tratto nero quelle risultate più attendibili: nella parte inferiore sono stati riportati, in percentuale, gli $A$ e i B relativi alle IV serie.

Dall'esame di questa figura risalta chiaramente la validità delle dromocrone calcolate negli studi condotti sui terremoti della Sicilia. In quasi tutti i terremoti analizzati, le percentuali degli A raggiungono valori elevati; spesso addirittura il $100_{i o}^{\circ}$ come nel caso dei terremoti provenienti da Gibilterra (n. 2), Marocco (n. 3), Algeria (n. 4), Tunisia (1. 7), Jugoslavia (n. 15), Grecia (n. 18), Creta (1n. 22); in quasi tutti gli altri terremoti si raggiunge il $100 \%$ con l' $(A+B)$.

Risultati un po' meno sodlisfacenti si sono ottenuti per alcuni terremoti provenienti dalla parte orientale del Mediterraneo: il n. 16, con epicentro in Jugoslavia ha solo il $20 \%$ di A e il $53 \%$ di B; la profondità controllata $(h=17,5 \mathrm{~km}$ ), l'uniea possibile, non risulta confermata dai controlli ed è probabile che non sia nemmeno attendibile,

(*) I'er esigenze tipografiche questa tabella è stata riclotta alla stampa delle parti essenziali (la testata e la parte conclusiva) tralasciando tutti gli elementi intermedi appena aceennati.

La tabella completa è tuttavia disponibile presso ggli antori. 


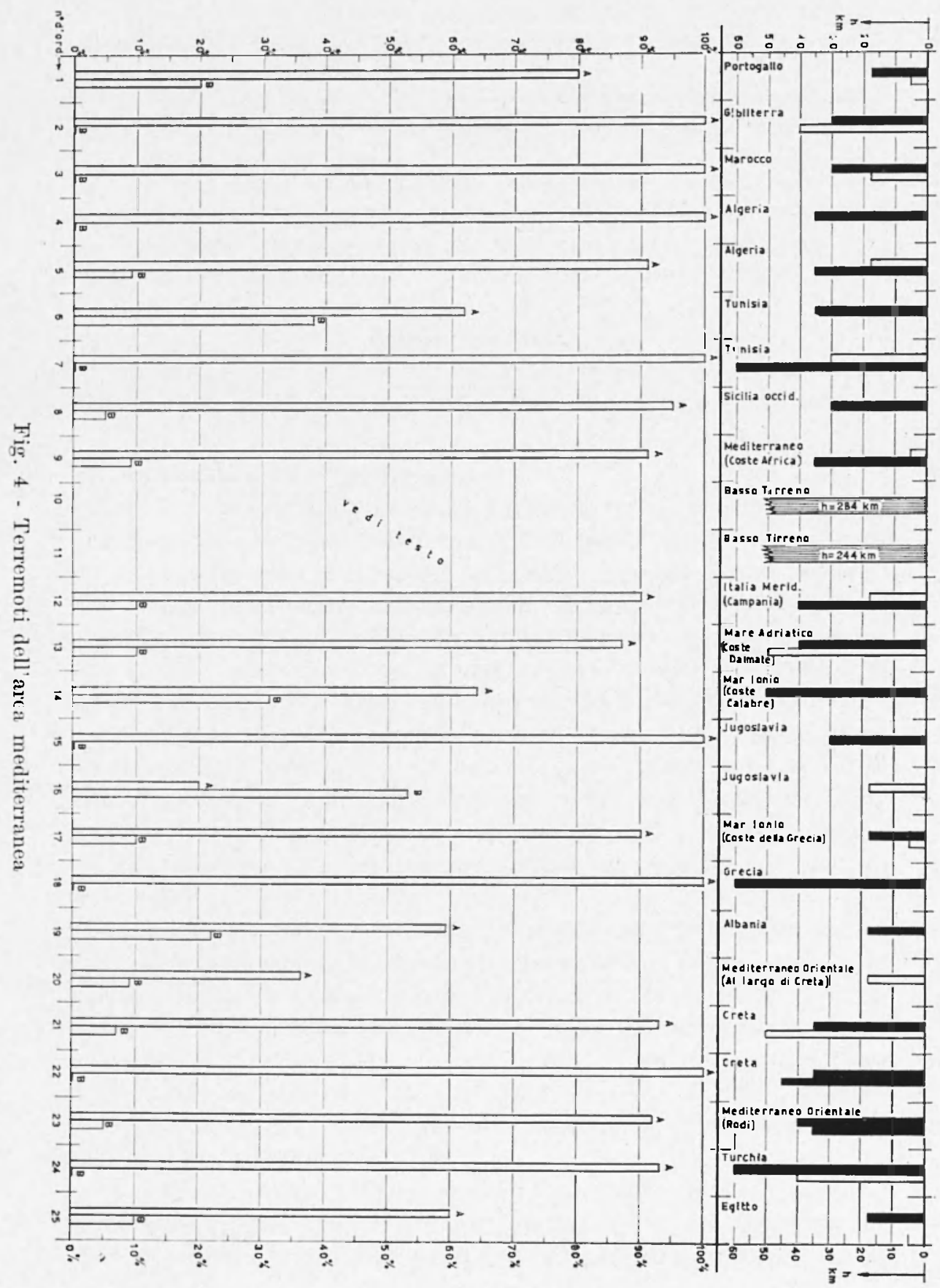


data la scarsita delle stazioni utilizzate dall'ISC per calcolarla (v. testo). Infatti il terremoto n. 15, con epicentro anchesso in Jugoslavia, a poca distanza dall'altro, ha fornito $\mathrm{mn}$ ottimo controllo per $h=30 \mathrm{~km}$ con le $A$ che raggiungono il $100 \%$.

Ancora un'altra profonditi non confermata si trova nel terr. 11. 20 (con epicentro al largo di Creta). $\mathrm{I} \cdot(A+B)$ non raggeiunge nemmeno il 50\% ed è da sospettare che anche questa profoncliti $(h=17,5 \mathrm{~km})$ pur essendo l'unica possibile, non sia in realtà quella più attendibile (v. osservazioni nel testo); i terremoti n. 21 e n. 22, pur essi con epicentro nei pressi di Creta, danno invece risultati di controllo molto sodalisfacenti per profondita maggiori $(h=35 \mathrm{~km}$ e $\tilde{\mathrm{o}}(\mathrm{km})$.

In conclusione si può dire che l'esame condotto sui 25 terremoti analizati confermi in maniera molto soddisfacente la validita delle curve di tragitto delle prime onde alle varie profondita ipocentrali da noi trovate nello studio sui terremoti della Sicilia occidentale; in particolare si nota che esse si possono usare con ma certa sicurezza anche per i teremoti provenienti dal bacino del Mediterraneo.

Questa ricerca ha messo in luce un altro aspetto importante: la maggior parte dei terremoti qui studiati presenta profondità ipocentrali molto prossime alla Moho; aleuni provengono addirittura dal Mantello Superiore: i terremoti del basso Tirreno, com'è risalputo, costituiscono una interessante anomalia nella struttura simotettonica cireostante, avvenendo a profondita ipocentrali che trovano riscontro solo in alcune regioni del Pacifico.

\section{BIBIIOGRAFIA}

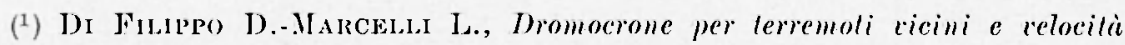
delle onde nell Italia Cembale. "Annali di Geofisica ", V, 2. 1952.

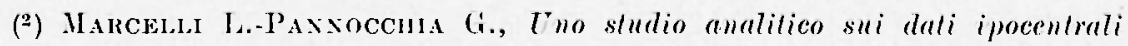
di 10 terremoli avvemuli in sicilia oceidentale nel Gennaio 1968. "Annali di Geofisica", XXIV, 2, 1971.

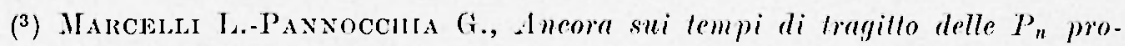
venienti dalla sicilia. Tabelle numeriche per varie projonditi ipocentrali. "Annali di Geofisiea", XXVII, 1-2, 1974.

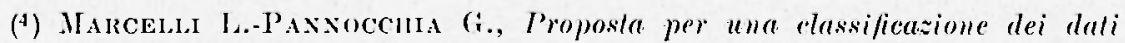
sperimentali in sismologia: clessi di allendibilitì $A, B,(, D$ in junzione dei $\delta$. 1 e dei $\delta 1$. "Annali di Geofisica", XXVII, 3-4, 1974. 
TABELLA 1

Riassuntiva dei terremoti studiati (Dati ipocentrali e classificezzione delle staziomi)

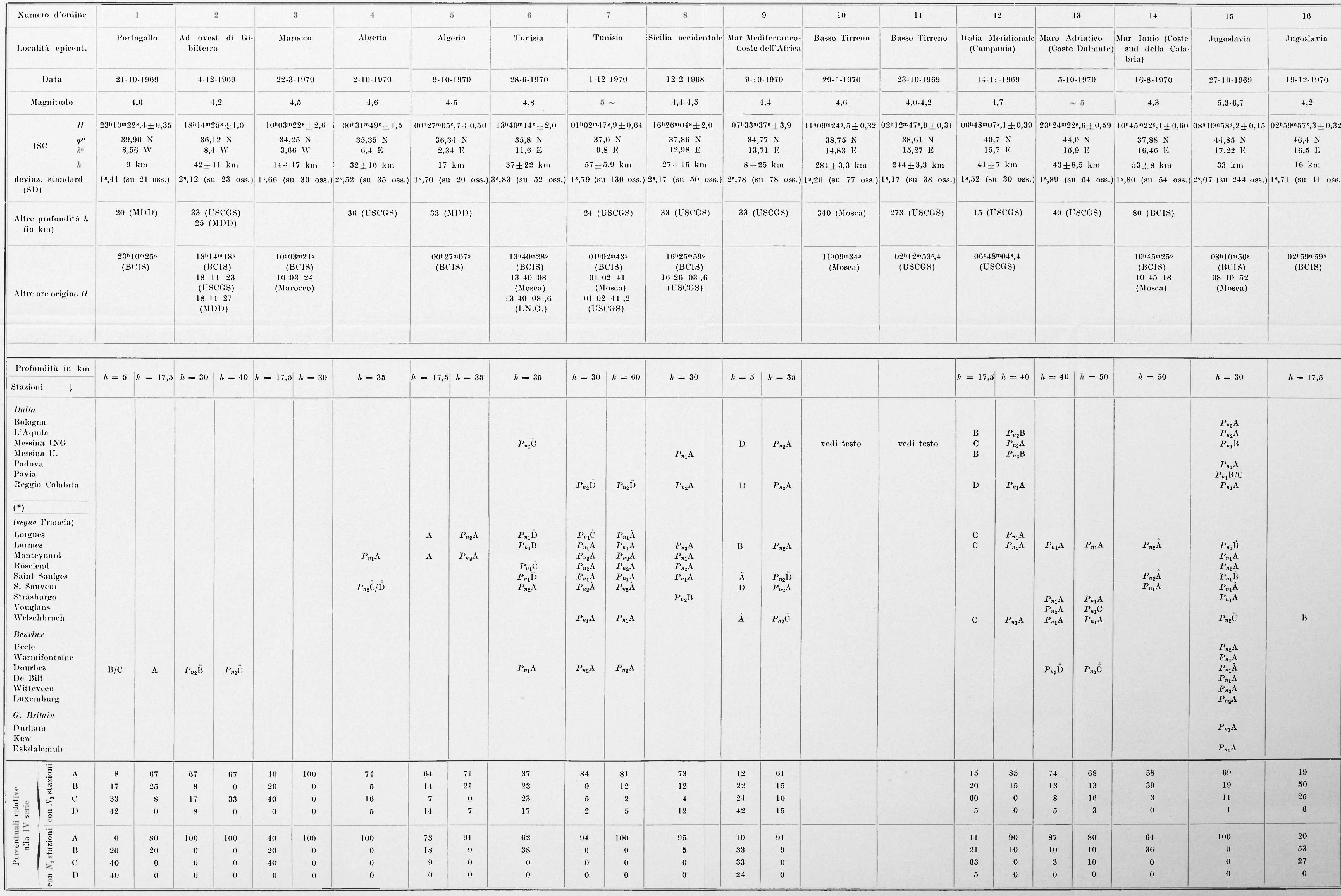

(*) Vedi nota a parg, 616 del testo. 


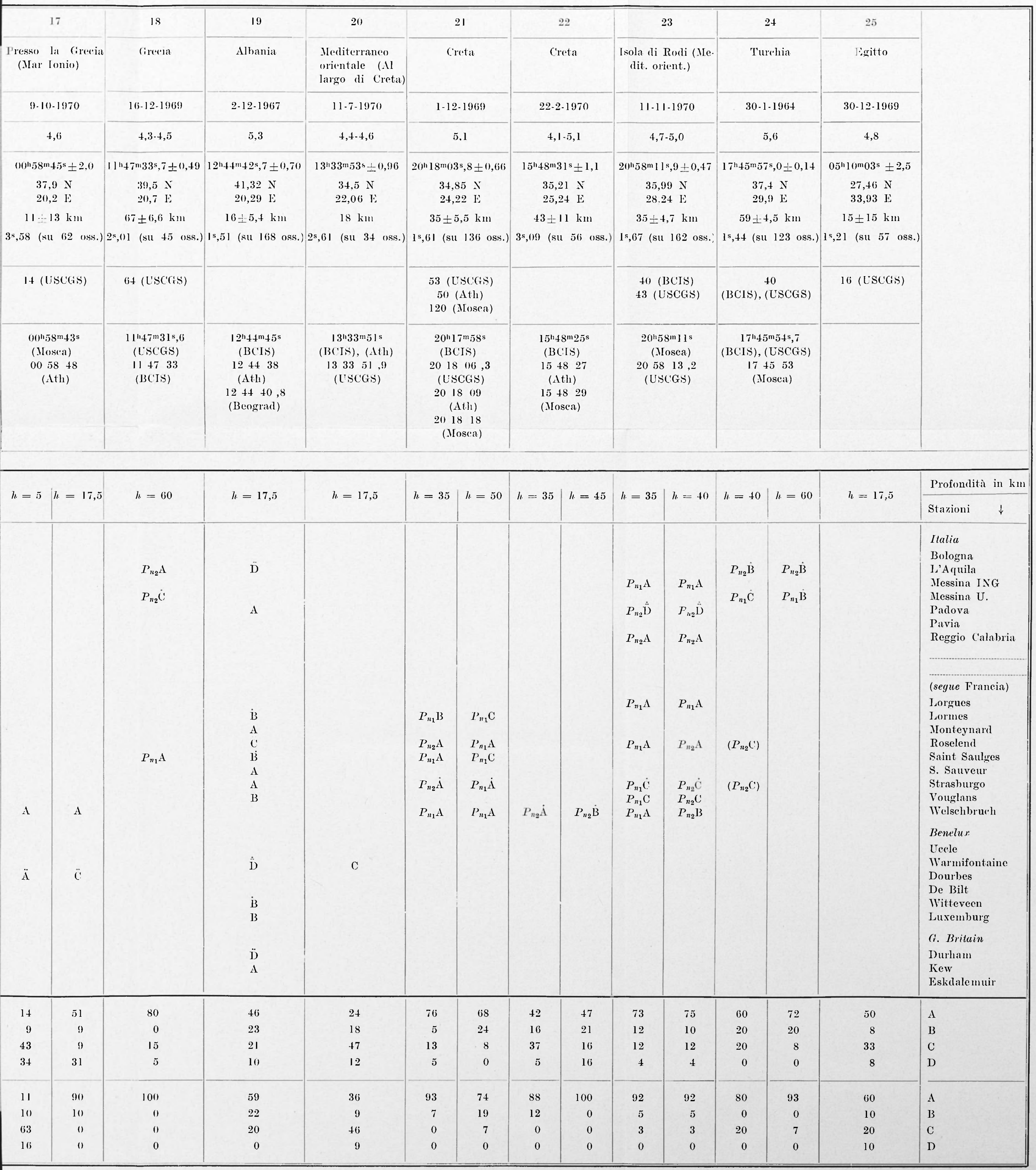


ABELISA

ndi (O.C) (ial bollett. I.S.C.)

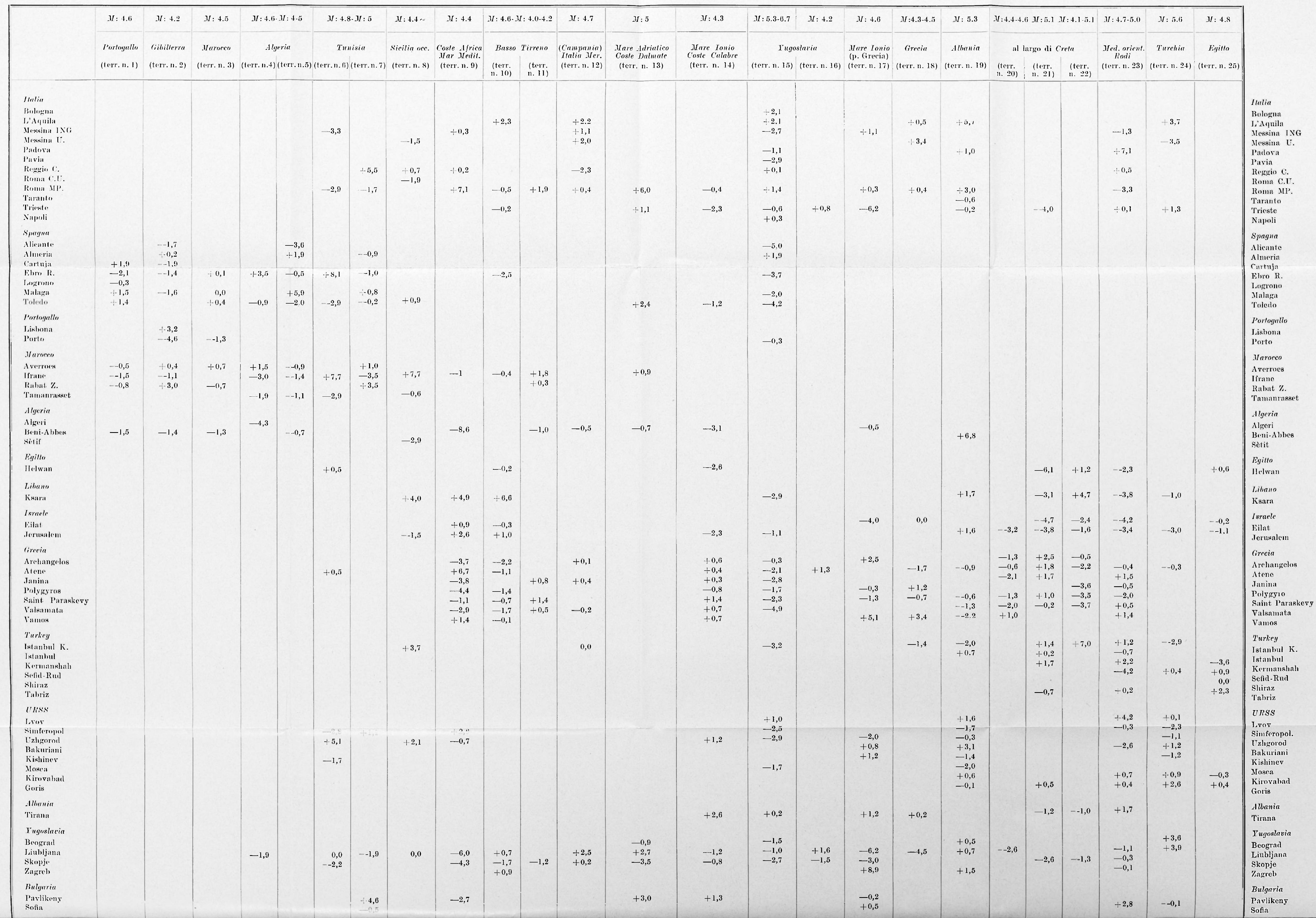


Engheria

Buclapest

Josvafo

P'isykesteto

Ceroselocacehia

Bratislava

Kasprerski: II.

P'rague

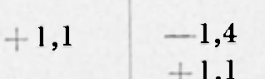

\section{Iolomia}

Crarovia

Nirolyic:a

\section{Allstria}

Vicuna K.

Germania

Benshergy

Buchlerlinedie

Colluberg

rimenonbere

Karlsrlue:

Moxa

Pruhonice

St utterart

'I'aunus

Joma

\section{Finlandia}

Nurmijarvi

\section{Danimarea}

copenhagen

Serzia

Virlelosolm

Ippsala

I'mera

\section{Snizzern}

Basilea

Francia

Foliniere (I a)

Garchy

Giomen

Isolat

I anslevillarl

I, Butcaux

Jorirues

lormes

Monteynarel

Rosclend

Saint Saulges

S. Sallvelur

straslurgo

Vouglans

Welschloruch

\section{Belyio}

Olauda

Uecelo

Warmifontaine

Dourbes

l)e Bilt

Wittrven

lurembourg

I,uxembourg

(i. Brilanmia

D) urham

Ke'w

Eskilalomuir

$$
-1,7
$$$$
+1,9
$$

$+1,9$

$-1,0$

$+0,7$

$-2,8$

$-1,4$

$$
\begin{array}{r}
-1,0 \\
+1,7
\end{array}
$$

$+3,5$

$+3,1$

$+5,7$

$-4,2$

$+1,0$

$+0,7$

$--0,9$

$-0,9$
$--0,8$

$-3,9$
$-2,0$

$--0,3$

$+0,9$

$--0,4$

$-6$

$-2,0$

$-3,6$

$-5,4$
$+1,9$

$+6,2$
$-3,4$

$--1,8$

$--1,3$

$--0,5$

$--0,5$

$+6, ?$

$+1,7$

$-0,9$

$--1,8$

$-2,2$

$+0,7$
+

$+5,5$

$+2,9$$$
-0,6
$$

$-0,6$
$+1,5$

$-2,2$
$-1,1$

$-0,6$

$+3,8$

$-2,3$

$+0.1$

$-0,5$

$-1,0$

1,4
$+1,4$

$\begin{array}{r}+1,4 \\ +2,0 \\ \hline\end{array}$

$-1,8$
$+2,5$

$-1,5$

$-1,4$

$+1,0$

$-0,1$

$-0,7$

$-0,3$

$--0,5$

$$
\begin{array}{r}
+1,7 \\
+1,7
\end{array}
$$

$-1-0,3$

$+1,5$

$+1,5$

$+1,3$

$-0,9$

$+1,1$
$-0,8$

$+0,6$

$$
\begin{array}{r}
0,6 \\
-0,2
\end{array}
$$

$-0,8$

$+0,4$

\section{$-2,8$}

$+0,6$

$+1,0$

$+0,4$

$-0$

$-0,3$

$+4,2$

$-1,0$

$-0,5$

$--1,3$

$+2,3$$$
\begin{array}{r}
+4 \\
-1
\end{array}
$$$$
-1,7
$$

$+3,1$

$-0,8$

$+0,4$

$-1,2$

$-1$

$-1,3$

$-1,7$

$-0,9$

$+1,5$

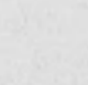

+0 ,

$+1,7$

$-1,7$

$+1$

$-1,2$

$-1,6$

$+3,2$

$+0,9$

$--0,8$

$--0,6$ 
